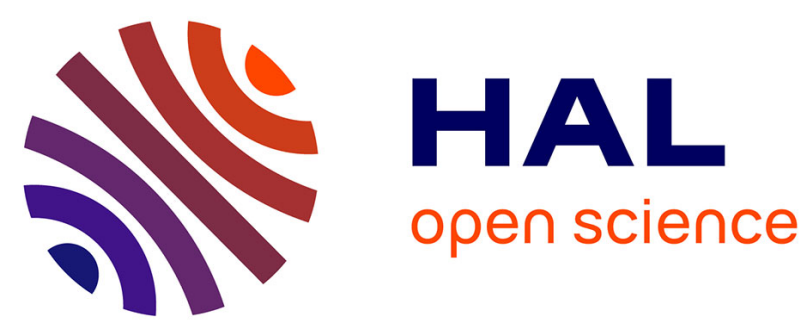

\title{
Beyond Euler-Cauchy Continua: The structure of contact actions in N-th gradient generalized continua: a generalization of the Cauchy tetrahedron argument.
}

\author{
Francesco Dell'Isola, Pierre Seppecher, Angela Madeo
}

\section{- To cite this version:}

Francesco Dell'Isola, Pierre Seppecher, Angela Madeo. Beyond Euler-Cauchy Continua: The structure of contact actions in N-th gradient generalized continua: a generalization of the Cauchy tetrahedron argument.. Variational Models and Methods in Solid and Fluid Mechanics, Volume 535 of the series CISM Courses and Lectures, pp.17-106, 2012. hal-00601624

\section{HAL Id: hal-00601624 \\ https://hal.science/hal-00601624}

Submitted on 19 Jun 2011

HAL is a multi-disciplinary open access archive for the deposit and dissemination of scientific research documents, whether they are published or not. The documents may come from teaching and research institutions in France or abroad, or from public or private research centers.
L'archive ouverte pluridisciplinaire HAL, est destinée au dépôt et à la diffusion de documents scientifiques de niveau recherche, publiés ou non, émanant des établissements d'enseignement et de recherche français ou étrangers, des laboratoires publics ou privés. 


\title{
Beyond Euler-Cauchy Continua: The structure of contact actions in N-th gradient generalized continua: a generalization of the Cauchy tetrahedron argument.
}

\author{
Francesco dell'Isola ${ }^{*}+$ and Pierre Seppecher ${ }^{\dagger+}$ and Angela Madeo ${ }^{\ddagger+}$ \\ * Università di Roma "La Sapienza" Dipartimento di Ingegneria Strutturale e \\ Geotecnica, Laboratorio Strutture e Materiali Intelligenti. email: \\ francesco.dellisola@uniroma1.it \\ † Institut de Mathématiques de Toulon U.F.R. des Sciences et \\ Techniques,Université de Toulon et du Var \\ ¥ Université de Lyon, Institut National des Sciences Appliquées (INSA) GCU et \\ LGCIE \\ + International Research Centre on "Mathematics \& Mechanics of Complex \\ Systems" M\& MOCS, Cisterna di Latina. \\ This work is dedicated to Professor Antonio Romano in occasion of his 70-th \\ birthday.
}

\begin{abstract}
The most general and elegant axiomatic framework on which continuum mechanics can be based starts from the Principle of Virtual Works (or Virtual Power). This Principle, which was most likely used already at the very beginning of the development of mechanics (see e.g. Benvenuto (1981), Vailati (1897), Colonnetti (1953), Russo (2003)), became after D'Alembert the main tool for an efficient formulation of physical theories. Also in continuum mechanics it has been adopted soon (see e.g. Benvenuto (1981), Salençon (1988), Germain (1973), Berdichevsky (2009), Maugin (1980), Forest (2006)). Indeed the Principle of Virtual Works becomes applicable in continuum mechanics once one recognizes that to estimate the work expended on regular virtual displacement fields of a continuous body one needs a distribution (in the sense of Schwartz). Indeed in the present paper we prove, also by using concepts from differential geometry of embedded Riemanniam manifolds, that the Representation Theorem for Distributions allows for an effective characterization of the contact actions which may arise in $N-t h$ order strain-gradient multipolar continua (as defined by Green and Rivlin (1964)), by univocally distinguishing them in actions (forces and $n-t h$ order forces) concentrated on contact
\end{abstract}


surfaces, lines (edges) and points (wedges). The used approach reconsiders the results found in the pioneering papers by Green and Rivlin (1964)-(1965), Toupin (1962), Mindlin (1964)-(1965) and Casal (1961) as systematized, for second gradient models, by Paul Germain (1973). Finally, by recalling the results found in dell'Isola and Seppecher (1995)-(1997), we indicate how Euler-Cauchy approach to contact actions and the celebrated tetrahedron argument may be adapted to $N$-th order strain-gradient multipolar continua.

\section{Introduction}

In a forthcoming review paper the authors will try to describe why, how and when many theories were conceived to go beyond the conceptual framework established for continuum mechanics in the Euler and Cauchy era. In this introduction are formulated only few comments about some papers which seem to be the starting point of the most modern studies in continuum mechanics. In this field -among the many available in the literature- the textbooks which we have found more instructive are those of Paul Germain and Jean Salençon. In them, without any loss of mathematical rigour, what nowadays seems the most effective approach to the axiomatization of mechanics is presented to the students of the École polytechnique. This approach is based on the Principle of Virtual Works. Paul Germain research paper on second gradient continua (1972) shows how fruitful is the aforementioned approach. Most likely one of the most illuminating paper in modern continuum mechanics is due to Green and Rivlin (1964): it is entitled "Simple force and stress multipoles" and formulates what the authors call "multipolar continuum mechanics". Indeed Green and Rivlin start there the foundation of what has been later called also the theory of generalized continua. They also address the problem of establishing simultaneously the bulk evolution equations and the correct boundary conditions for generalized continua: equations and boundary conditions which they find by postulating the Principle of Virtual Work. In this aspect their theory is perfectly orthodox with respect to the paradigm put forward, many years earlier, by Cosserat brothers, in their fundamental textbooks (1908)-(1909). Of great importance for understanding the relationship between Cosserat continua and higher order gradient continua studied by Green and Rivlin is the short but very clear paper by Bleustein (1967), where the boundary conditions found by Toupin in a previous work are interpreted also from a physical point of view. We must also cite here the papers by Mindlin, who also contributed greatly to the development of important generalizations of Euler-Cauchy continuum models. In particular in Mindlin (1965) is started the study of third gradient continua, which is developed in a great extent. 
However in all cited papers the Cauchy cuts considered are very regular: therefore the cited authors refrain from the consideration of contact actions concentrated on edges and wedges. Instead Germain considers Cauchy cuts in which the normal can suffer discontinuities of the first kind: he therefore needs to consider contact actions concentrated on edges. However Germain limits his treatment to second gradient continua: in his theory there are not wedge contact actions. Also in Toupin's strain-gradient theory (1962) the consideration is limited to second gradient continua. In cited paper Toupin limits himself to the consideration of a particular class of second gradient continua: those in which only a particular class of contact double-forces (using the nomenclature by Germain) can be exerted: the class constituted by "couple-stresses".

Those which are called by many authors (see e.g. Maugin (2010) and Forest (2005)-(2006)) generalized continua actually strictly include higher gradient continua which we consider here. In generalized continua together with the placement field one can introduce many other kinematical descriptors, which are other fields defined in the material configuration of considered continuum. The first example of such a set further kinematical descriptors is given by Cosserats who add to the placement field also a field of "changes of attitude", i.e. a field of rotations, which describe a large class of "microscopically structured" continua. As clarified by Bleustein (1967) Toupin's continua are a class of Cosserat continua in which an internal constraint has been introduced. In the following sections, while commenting some papers recently published in the field, it is discussed how the approach used by Germain can be reconciled with an approach which parallels more strictly the one used, for first gradient continua, by Cauchy.

It is not easy (but this analysis will be attempted in the aforementioned review paper) to explain why the foundation of continuum mechanics "à la Cauchy" has been considered "more physically grounded" than the axiomatization based on the Principle of Virtual Powers. In the present work we prove that at least for higher gradient continuum theories, the two approaches are completely equivalent.

Variational Principles and Calculus of Variations have always been an important tool in formulating mathematical models for physical phenomena. Among many others the textbook by Berdichevsky (2009) clearly shows that this statement holds, in particular, for Continuum Mechanics.

We are sure that the Principle of Virtual Works and the Principle of Least Action have roots much deeper than many scientists believe. (see e.g. Vailati (1897)). One can conjecture that the majority of physical theories were first formulated in terms of these Principles, and only subsequently reconsidered from other points of view. The Principle of Least Action, which 
supplies a "geometric" (see Russo (2003)) version of mechanics, is likely to be indeed the tool used by the true founders of mechanics (i.e. the scientists of the Hellenistic period) to establish it. As conjectured also by Colonnetti (1953) and Rorres (2004) Archimedes himself was basing his mechanical investigations on the Principle of Virtual Works. More recently, as already stated above in a more detailed way, Green, Rivlin, Toupin, Mindlin, Casal, and Germain formalized various versions of the theory of generalized continua basing them on the Principle of Virtual Powers: however the most illuminating treatises in this subject remain those due to Cosserat brothers (1908)-(1909).

For a long time some opponents to second gradient theories argued about its "lack of consistency", due to the difficulties in "interpreting" boundary conditions. However it has to be remarked that if one refuses to use the Principle of Virtual Powers he can find very difficult the job of finding some set of boundary conditions which are compatible with the (independently postulated!) bulk evolution equations. Actually it happens that many epigones, after having initially refused to use this principle also in continuum mechanics, have later rephrased with different notations many of the results already available in the literature.

If instead one accepts the D'Alembertian approach to mechanics all these problems of well-posedness of mathematical models completely disappear.

\section{Second and Higher Gradient Continuum Theories}

In the last fifty years it has been widely recognized that in order to describe a wealth of physical phenomena it is needed to introduce mechanical theories which take into account contact actions more complex than those considered in the format given by Cauchy to continuum mechanics. Some well-known contributions in this regard are given in the papers listed in the references by Toupin, Mindlin, Green, Rivlin, Maugin, Forest, Germain, Suiker, Sokolowski, Triantafyllidis among many others.

More recently it has been recognized that second or even higher gradient models are needed when continuum models are introduced for describing systems in which strong inhomogeneities of physical properties are present at eventually different length scales (see e.g. Abu et al. (2008), Alibert et al. (2003), Polizzotto (2007), Pideri and Seppecher (1997), Trianttafyllidis et al. (1986)-(1998), Yang and Misra (2010), Yang et al. (2011)), and may be of great importance also in continuum systems in which some "microscopical" degrees of freedom can "capture" a relevant amont of deformation energy (see e.g. Carcaterra (2005) or Carcaterra et al. (2006)).

Actually, immediately after the development of the Cauchy format of 
continuum mechanics, a first relevant generalization in the aforementioned direction was conceived by Eugène and François Cosserat, but their efforts were not continued until late in XX century. Cosserat described continuum bodies in which contact actions were to be modelled not only by means of surface forces, but also by means of surface couples. The conceptual differences between Cauchy-type continuum mechanics and Cosserat-type continuum mechanics were relevant, and the second one could not be obtained by means of simple modifications of the first one. The remarkable mathematical difficulties confronted by Cosserat rendered their work difficult to be accepted, and for a long period their results were nearly completely ignored. This circumstance can be easily understood: the structure of Cosserat contact actions is complex. Indeed in Cosserat continua one needs, together with Cauchy stress tensor also a Couple stress tensor, for representing contact Couples.

\subsection{A first method for extending Cauchy model for continuous bodies}

In order to develop continuum mechanics by going beyond the Cauchy format it is possible to use at least two different approaches.

The most simple of them, used also by Cosserats, starts by postulating how the power expended by internal actions in a body depends on the "virtual" velocity field and its gradients. Starting from this postulate one can deduce, by means of a successive application of the theorem of divergence, i.e. by means of several iterative integrations by parts, which are the contact actions which can be exerted at the boundary of the considered body. Hence, this method starts from the notion of stress tensors and deduces from it the concept of contact actions. It is based on the D'Alembert Principle of Virtual Work and has been resumed by Green and Rivlin, Mindlin, Casal and subsequently by Paul Germain, in his enlightening papers (1972-1973). This Principle is undoubtedly a great tool in Mechanics which has not been improved since its original first and "standard" formulation, differently to what stated in Fried and Gurtin (2006)-(2008) and in Podio-Guidugli (2009). It is not clear why these last authors consider as "non-standard" a formulation of the Principle of Virtual Powers which can be found stated "word-for-word" for instance in the textbooks of Jean Salençon..

Indeed other authors (e.g. the paper by Degiovanni, Marzocchi, Musesti (1999)-(2010) in the references ) stated that:

In particular, the approach by means of the theory of distributions, mentioned by Germain himself but not fully developed, is here adopted from the beginning. Clearly, in order to obtain deeper results such as the Cauchy 
Stress Theorem, some extra regularity has to be assumed. Note that a power depends in general from two variables, the velocity field and the subbody. So it is a bit more complex than a mere distribution.

In the same spirit in dell'Isola and Seppecher (1995)-(1997) the starting assumptions concerning contact actions are: i) for every subbody of considered body the power expended by contact actions on a generic velocity field is a distribution (i.e. a linear and continuous functional on velocity fields) ii) the power expended by contact actions is quasi-balanced (generalizing the assumption used in Noll and Virga (1990)). Then in aforementioned papers by using different polynomial test velocity fields and different families of subbodies, the Cauchy construction for stress tensors is obtained.

The works of Green and Rivlin, Mindlin and Germain have been taken up again and again, (e.g. in Fried and Gurtin (2006)-(2008)) often rephrasing them without introducing any notable amelioration and often second gradient continua are somehow confused with Cosserat continua.

Paul Germain, following a tradition set in France by André Lichnerowicz, uses the original version (and more efficient) absolute notation due to Levi-Civita. This version, at least in this context, is the most adapted, as many objects of different tensorial order are to be simultaneously handled. Sometimes those who are refraining from using the most sophisticated version of Levi-Civita absolute Calculus are lead to refer to the needed stress tensors and the related contact actions indistinctly using the names "hyperstresses" and "hypertractions". On the contrary Germain (following the spirit of Green and Rivlin) tries to convey through the nomenclature chosen the physical meaning to be attached to the new mathematical objects which he is introducing: for instance he calls "double forces" the actions which are expending powers on the velocity gradient in the directions which are normal to the surfaces of Cauchy cuts. Germain then decomposes these "double forces" into "couples" and "symmetric double forces" recognizing (following Bleustein) that couples were already introduced by Cosserats. Germain's notation supports the mechanical and physical intuition contrarily to what does a generic nomenclature based on some "hyper" prefixes.

\subsection{A second method for extending Cauchy model and its rela- tionship with the first}

The second method starts by postulating the type of contact action which can be exerted on the boundary of every "regular" part of a body and then proceeds by proving a "representation" theorem for the considered class of contact actions: the existence of stress tensors is then deduced from the postulated form of contact actions with the addition of a "balance-type" 
postulate, based on physical grounds. In other words: to the "constitutive" assumption adopted for characterizing the class of contact action under consideration one must add a Principle of Balance: the contact actions have to be balanced by a bulk action. This is the method followed by Cauchy which is often considered as the only firm foundation of Continuum Mechanics. The important contribution due to Noll and Virga (1990) is to have introduced the assumption of "quasi-balance" for powers, which generalized, in the most suitable way, the Euler-Cauchy Postulate used in Cauchy continuum mechanics.

\section{The mathematical difficulties presented by this second method}

As remarked explicitly in dell'Isola and Seppecher (1995)-(1997) and in Degiovanni, Mazzocchi and Musesti (1999)-(2010) the mathematical difficulty to be confronted in order to establish a firm foundation for this second method relies on the dual dependence of power functional on velocity fields and on subbodies of the considered continuum. It is obvious, starting from physical plausibility considerations, that power functionals must be regarded as distributions on the set of test functions represented by the admissible velocity fields (see e.g. the textbooks of Salençon and Germain).

A fundamental results due to Schwartz allows for representing distributions (with compact support) as finite sums of derivatives of measures Schwartz (1973). When (as it is important for considering contact actions) the distribution is concentrated on a smooth submanifold of threedimensional Euclidean space, then the derivatives to be considered are only those "normal" or "transversal" to the submanifold itself. Unfortunately in Schwartz it is not considered a representation theorem for families of distributions "attached to" the family of measurable subset of a given measurable set.

Some of the efforts of Degiovanni, Marzocchi and Musesti (1999)-(2010), Lucchesi, Šilhavý and Zani (2008) are directed, with remarkable results, to the search of such a generalized Schwartz representation theorem and to the formulation of weaker versions of Gauss divergence Theorem.

Indeed it is also of relevance the problem arising when one must define generalized "stresses" having a flux which allows for the representation of contact action and a divergence to be used for formulating bulk "local" form of balance laws. This problem has been also addressed with some interesting results (see Lucchesi, Silhavý and Zani (2008), Degiovanni, Marzocchi e Musesti (1999), Šilhavý (1985)-(1991). 


\subsection{The two methods can be reconciled.}

During a long period the first method of the two previously described has been rejected by many researchers and it is lucky for advancement of science that its power has been, in the last decade, finally nearly unanimously accepted.

Moreover the two methods can be reconciled.

Indeed the equivalence of the two methods has been explicitly established by Cauchy him-self and made precise by Noll, for First Gradient Theories.

The same equivalence has been proven for the so called Second Gradient Theories, i.e. for theories in which the internal power is a second order distribution: this results has been obtained in the sequence of papers Noll and Virga (1990), dell'Isola and Seppecher (1995)-(1997). In these last two papers the relationship between the concept of contact line force and surface double forces was proven and also a representation formula relating the two kind of forces was obtained.

\section{Some commentaries about a recent paper on second gradient continua}

Unfortunately it seems that the fundamental connection between the two methods (and the available proof of the existence of this connection at least for those materials which were called by Germain second gradient materials) seems still not well understood in part of the mechanics community, while it has been considered as established by others (see e.g. in the references the works by Maugin, Markus and Forest).

- For instance, one can read in Podio-Guidugli Vianello (2010) that:

Although here we do not deal with this difficult issue directly, in Sect. 3, the bulk of this article, we do provide a full set of representation formulae not only, as is relatively easy, for tractions and hypertractions in terms of stresses and hyperstresses (see definition (26) for diffused tractions and hypertractions, and definition (27) for tractions concentrated on edges), but also, conversely, for stresses and hyperstresses in terms of diffused and concentrated tractions and hypertractions (see definitions (28, 29), and (34). Such representation formulae generalize the corresponding formulae for simple (!first-gradient) materials, that we derive in our preparatory Sect. 2. Since we work in a nonvariational setting, our results apply whatever the material response. The PVP we use includes edge tractions, both internal and external; without them, it would not be possible to arrive at the complete representation formula for the hyperstress in terms of hypertractions we construct in Sect. 3.5. 
However it is not possible to see any difference between the results listed in the previous statement and those obtained by Casal and then by Germain. The quoted results are also available in Seppecher (1987)-(1989), Casal and Gouin (1985) and even recalled in the textbooks by Forest. An interesting application of second gradient theories to the mechanics of porous media are proposed e.g. in Collin et al. (2006) or in Sciarra, dell'Isola and Coussy (2007), where many results listed in the commented paper are explicitly exploited.

- In the commented one then reads:

An interesting feature of second-gradient materials is that, if bodies and subbodies having non everywhere smooth boundary are considered, then edge forces, i.e., line distributions of hypertractions are to be expected (and, if a dependence on gradients higher than two is allowed, one has to deal also with vertex forces, as exemplified by Podio-Guidugli [15]). To our knowledge, a rigorous interaction theory accommodating such a nonstandard behavior remains to be constructed; interesting attempts in this direction have been carried out by Forte and Vianello [3], Noll and Virga [14], and Dell'Isola and Seppecher [1].

This statement is not accurate: indeed the efforts leading to in the papers Noll and Virga (1990), dell'Isola and Seppecher (1995)-(1997), as recognized for instance in the works by Maugin and Forest cited in the references, actually constructs the searched rigorous interaction theory.

- Again in the commented paper the following statement can be found:

Finally, in Sect. 4, we provide a new proof of the following not very well-known fact in the theory of second-gradient materials: if edge tractions are constitutively presumed null on whatever edge, then the hyperstress takes a very special form whose information content is carried by a vector field. We surmise that inability to develop edge interactions be characteristic of certain second-gradient fluids, an issue that we take up in a forthcoming article [17], continuing a line of thought proposed by Podio-Guidugli [16].

Indeed, this results, rather obvious, is obtained in dell'Isola and Seppecher (1997), Remark 3, page. 48 and systematically exploited in the application of second gradient theory presented in Sciarra et al. (2001)-(2008) or in Madeo et al. (2008). Some interesting consideration about this point are already available in Seppecher (1987) together with some consideration about third gradient fluids. This result is well-known: for instance remark that Equation (35) on page. 173 in the commented paper exactly is equal to Equation (18) page. 6612 in Sciarra et al. (2007) or to Equation (13) pag.107 in Sciarra et al. (2008). 
- Finally always in the commented paper one reads

Relations (7) and (8) are also arrived at when, as is customary, only tractions on body parts are introduced, because stress is constructed à la Cauchy as a consequence of balance of tetrahedron-shaped parts. The Cauchy construction is the pillar on top of which the standard theory of diffuse (i.e., absolutely continuous with respect to the area measure) contact interactions stands. For complex (i.e., nonsimple) material bodies, a Cauchy-like construction has been attempted often, but not achieved so far, to our knowledge.

On the contrary in the Conclusions of the paper dell'Isola Seppecher (1997) one can read:

The most important concepts introduced in this paper are:

(i) the concept of quasi-balanced power of contact force distribution and

(ii) that of prescribed shapes.

They allowed us to develop a system of axioms "à la Cauchy' for continua in which edge contact forces are present.

It is not clear if the authors of the commented paper are completely aware of this last statement and of the assumptions and theorems presented in the just cited paper. In our opinion, the demanded Cauchy-like construction for second gradient materials is supplied there.

\subsection{Concluding remarks}

The connection between internal power and the power expended by external actions has not been yet completely established for a generic $N$-th Gradient Theory, although interesting and useful considerations can be found in the papers by Green, Rivlin, Mindlin, Di Carlo and Tatone and Podio-Guidugli cited in the references.

In the following sections it will be shown how the work started in dell'Isola Seppecher (1997) can be continued. The aim in these lecture notes will be to give a firm framework to those researchers which need to deal with more complex contact actions (for instance "wedge forces"), wish to refrain from using the Principle of Virtual Power and instead prefer to adopt an approach based on "contact interactions" rather than on "virtual power expended on virtual velocity fields".

Indeed the ideas presented in the just mentioned paper can be extended rather easily to treat the case of all types of contact distributions: more precisely the Cauchy tetrahedron argument can be generalized to prove that all types of mechanical contact actions can be represented in terms of a generalized stress tensors.

To our knowledge the results which we present are novel. It is however 
difficult to establish how many of them were already obtained in the literature: in general while trying to write a scientific work and in particular when revisiting Cauchy's, Green's, Rivlin's and Germain's results it is advisable to try to avoid the attitude of the novelist Pierre Menard (see Borges):

(...) Menard's visible work can be easily enumerated. Having examined with care his personal files, I find that they contain the following items: (a list of works follows) I turn now to his other work: the subterranean, the interminably heroic, the peerless. And-such are the capacities of man! - the unfinished. This work, perhaps the most significant of our time, consists of the ninth and thirty-eighth chapters of the first part of Don Quixote and a fragment of chapter twenty-two. I know such an affirmation seems an absurdity; to justify this "absurdity" is the primordial object of this note. (....) He did not want to compose another Quixote - which is easy - but the Quixote itself. Needless to say, he never contemplated a mechanical transcription of the original; he did not propose to copy it. His admirable intention was to produce a few pages which would coincide-word for word and line for line-with those of Miguel de Cervantes. "My intent is no more than astonishing," (...) To be, in some way, Cervantes and reach the Quixote seemed less arduous to himand, consequently, less interesting - than to go on being Pierre Menard and reach the Quixote through the experiences of Pierre Menard. (....) "My undertaking is not difficult, essentially," I read in another part of his letter. "I should only have to be immortal to carry it out."

\section{Resumé of some results in Differential Geometry of Riemannian manifolds embedded in the Euclidean Space.}

The reference configuration of the continuum which we want to consider is a regular region $C^{*}$ embedded in $E^{3}$. Its boundary $\partial C^{*}$ is assumed to be a piecewise regular orientable and rectifiable surface.

Following the ideas already expressed in Cosserat (1908) and formalized e.g. by Germain (1972) or Salençon (1988-2005) we will assume that the Principle of Virtual Powers holds at least for every "regular" subbody of the considered body. In this section we specify what we mean with "regular" subbody and in particular we specify how regular must be its topological boundary. 


\section{Gaussian Geometry of Piecewise Regular Surfaces in $E^{3}$}

The family of subbodies which we will consider in the Principle of Virtual Powers will have a topological boundary which is a piecewise regular surface, as defined in the following section. These surfaces represent a particular class of "shapes" as introduced in dell'Isola and Seppecher (1995)-(1997).

\subsection{Piecewise regular surfaces embedded in $E^{3}$.}

In the present paper we will use the following nomenclature:

Definition 5.1. A surface $S$ is a piecewise regular (orientable and rectifiable) surface embedded in $E^{3}$ when there exist a finite set

$$
\left\{\gamma_{i} \subset S, i=1, \ldots N\right\}
$$

of $C^{1}$ curves (called edges) and a finite set of points (called wedges)

$$
\left\{W_{i} \in S, i=1, \ldots, M\right\}
$$

such that, once introduced the notation (which is reminiscent of the one usually encountered in the formulation of Poincaré theorem for exterior forms)

$$
\begin{aligned}
\operatorname{Support}(\partial S) & :=\left(\bigcup_{i=1}^{N} \gamma_{i}\right) ; \\
\text { Support }(\partial \partial S) & :=\left(\bigcup_{i=1}^{M} W_{i}\right),
\end{aligned}
$$

the conditions appearing in the following list are verified.

\section{LIST OF CONDITIONS}

- for every $p \in S-(\partial S \cup \partial \partial S)$ (i.e. for every regular point belonging to $S$ ) there exists a neighborhood in $S$ which is locally $\left(C^{2}-\right)$ diffeomorphic to $\mathbb{R}^{2}$ : we call any such local diffeomorphism

$$
r: \mathbb{R}^{2} \rightarrow S-(\partial S \cup \partial \partial S)
$$

an internal chart of $S$, We assume that for every internal chart $r$ the set $r\left(\mathbb{R}^{2}\right)$ is a rectifiable surface; 
- for every $p \in S-(\partial S \cup \partial \partial S)$ there exists a translation vector in $E^{3}$, denoted with the symbol $N(p)$, which is orthogonal to every tangent vector to $S$,

- for every $p \in \partial S-\partial \partial S$ there exist two diffeomorphisms (called also "border charts")

$$
r^{ \pm}:\left[0, \infty\left[\times \mathbb{R} \rightarrow I^{ \pm} \subset S\right.\right.
$$

such that

$$
\begin{gathered}
r^{ \pm}(0,0)=p ; \quad r^{ \pm}(0, \mathbb{R})=\partial S \cap I^{ \pm} \\
;(\forall y \in] 0, \infty[\times \mathbb{R})\left(r^{ \pm}(y) \in S-(\partial S \cup \partial \partial S)\right)
\end{gathered}
$$

and both the following limits exist

$$
\lim _{x \rightarrow(0,0)} N\left(r^{ \pm}(x)\right):=N^{ \pm}(p) .
$$

Therefore every curve $\gamma_{i}$ can be regarded as the border of two regular surfaces $S^{ \pm}$one on the side + the other on the side - with respect to $\gamma_{i}$. We will denote the unit outward pointing normal vector to $\gamma_{i}$ in the tangent plane to $S^{ \pm}$respectively with the symbol $\nu^{ \pm}$.

- for every curve $\gamma_{i}$ (the length of which is denoted by $l_{i}$ ) there exists a global parametric $C^{1}$ representation $r_{i}$

$$
r_{i}: s \in\left[0, l_{i}\right] \mapsto p \in S
$$

such that

$$
\left\|\frac{d r_{i}}{d s}\right\|=1, \frac{d r_{i}}{d s} \cdot N^{ \pm}=0 .
$$

We will assume that

$$
\frac{d r_{i}}{d s} \times N^{ \pm}= \pm \nu^{ \pm} .
$$

- for every $W_{j} \in \partial \partial S$ there exists at least one curve $\gamma_{i}$ such that one of the two following conditions holds

$$
r_{i}(0)=W_{j} \text { or } r_{i}\left(l_{i}\right)=W_{j} .
$$

Roughly speaking piecewise regular orientable and rectifiable surfaces, in the particular conventional sense specified above, are surfaces where the normal vector is defined in all points except those belonging to a finite set of wedges and to a finite set of regular curves (the edges of the surface). Along these curves a tangent vector is always defined together with both the normals of the two subsurfaces concurring on the edge.

Definition 5.2. We call face of $S$ every connected component of the set $S-(\partial S \cup \partial \partial S)$ 


\subsection{Local parametrizations for regular curves and surfaces. Lo- cal curvilinear coordinate systems in $E^{3}$ adapted to surfaces and surface edges.}

We start recalling some basic definitions from differential geometry

Definition 5.3. A parametrization of a regular curve is a $C^{1}$ one-to-one function $r:] a_{1}, b_{1}\left[\rightarrow E^{3}\right.$ such that

$$
\left(\forall p \in r(] a_{1}, b_{1}[)\right)\left(t(p):=\frac{d r}{d x^{1}}\left(r^{-1}(p)\right) \neq 0\right) .
$$

The vector $t(p)$ is the tangent vector to the curve in $p$.

Definition 5.4. At every point $p$ of a regular curve $\gamma$ we can define the following projection operators

$$
P_{\gamma, p}:=t(p) \otimes t(p) ; \quad Q_{\gamma, p}:=N_{1}(p) \otimes N_{1}(p)+N_{2}(p) \otimes N_{2}(p)
$$

where $N_{1}(p)$ and $N_{2}(p)$ form an independent set of vectors both orthogonal to $t(p)$.When this will not cause confusion we will skip one or both the indices of the introduced projectors. $P_{\gamma, p}$ will be called the projector in the tangent bundle (or line) of $\gamma$ at point $p$, while $Q_{\gamma, p}$ will be called the projector in the orthogonal bundle (or plane) of $\gamma$ at point $p$.

Definition 5.5. A map

$$
\left.r:\left(x^{1}, x^{2}\right) \in\right] a_{1}, b_{1}[\times] a_{2}, b_{2}\left[\subset \mathbb{R}^{2} \mapsto r\left(x^{1}, x^{2}\right) \in E^{3}\right.
$$

is called a local parametrization for the regular surface $S$ in the neighborhood of $p$ if $r$ is a $C^{1}$ diffeomorphism between $] a_{1}, b_{1}[\times] a_{2}, b_{2}[$ and $r(] a_{1}, b_{1}[\times] a_{2}, b_{2}[)=: I_{p}$ such that

$$
\begin{array}{r}
r(] a_{1}, b_{1}[\times] a_{2}, b_{2}[)=: I_{p} \subset S ; \\
\left.r^{-1}(p) \in\right] a_{1}, b_{1}[\times] a_{2}, b_{2}[
\end{array}
$$

Once a local parametrization for the regular surface $S$ is introduced then a set of coordinate curves on $S$ is established, together with a field of bases for the tangent planes to $S$.

We will consider in the following the fields of vectors induced by a local parametrization

$$
(\forall \alpha \in\{1,2\})\left(\forall q \in I_{p}\right)\left(a_{\alpha}(q):=\frac{\partial r}{\partial x_{\alpha}}\left(r^{-1}(q)\right)\right) .
$$


The couple $\left\{a_{1}(q), a_{2}(q)\right\}$ is a basis of the tangent plane to $S$ in the point $q$. The set of points

$$
r\left(\left\{x^{1}\right\} \times\right] a_{2}, b_{2}[), r(] a_{1}, b_{1}\left[\times\left\{x^{2}\right\}\right)
$$

are called the coordinate $x^{2}$ and $x^{1}$ curves. Remark that the vectors $a_{\alpha}$ are tangent to the coordinate $x_{\alpha}$ curves. The $C^{1}$ normal (to $S$ ) unit vector field $N$ can be calculated by the formula

$$
N(q)=\frac{a_{1}(q) \times a_{2}(q)}{\left\|a_{1}(q) \times a_{2}(q)\right\|}
$$

Definition 5.6. Let the couple $\left\{a_{1}(p), a_{2}(p)\right\}$ denote an othonormal basis of the tangent plane to $S$ in the point $p$. At every point $p$ of a regular surface $S$ we can define the projector operators

$$
\begin{gathered}
P_{S, p}:=a_{1}(p) \otimes a_{1}(p)+a_{2}(p) \otimes a_{2}(p) ; \\
Q_{S, p}:=N(p) \otimes N(p) .
\end{gathered}
$$

When this will not cause confusion we will skip one or both the indices of the introduced projectors. $P_{S, p}$ will be called the projector in the tangent bundle (or plane) of $S$ at point $p$, while $Q_{S, p}$ will be called the projector in the ortogonal bundle (or line) of $S$ at point $p$.

Remark 5.7. Because of the previous definitions, regular curves and surfaces are respectively one dimensional and two dimensional manifolds embedded in the three dimensional Euclidean space.

The scalar fields

$$
g_{\alpha \beta}: q \in I_{p} \mapsto a_{\alpha}(q) \cdot a_{\beta}(q)
$$

represent the components of a tensor field which is called the Riemannian metric induced on $S$ by the inner product in $E^{3}$.

Remark 5.8. A given regular curve or surface can be endowed with the structure of Riemannian manifold simply using the inner product of the Euclidean space in which they are embedded. Indeed for any couple $v$ and $w$ of vectors in their tangent bundle one can calculate their inner product simply by regarding them as vectors in $E^{3}$.

Although in the Euclidean space $E^{3}$ the Cartesian system of coordinates, using at every point the same vector basis to represent displacement vectors, is in general sufficient, in the present context one needs to introduce the following 
Definition 5.9. Local curvilinear coordinate systems in $E^{3}$. A map

$$
\left.\varphi:\left(x^{1}, x^{2}, x^{3}\right) \in\right] a_{1}, b_{1}[\times] a_{2}, b_{2}[\times] a_{3}, b_{3}\left[\subset \mathbb{R}^{3} \mapsto \varphi\left(x^{1}, x^{2}, x^{3}\right) \in E^{3}\right.
$$

is called a local chart in $E^{3}$ in the neighborhood $I_{p}$ of $p \in E^{3}$ determining a local curvilinear coordinate system when it is a diffeomorphism between

$$
] a_{1}, b_{1}[\times] a_{2}, b_{2}[\times] a_{3}, b_{3}\left[\text { and } \varphi(] a_{1}, b_{1}[\times] a_{2}, b_{2}[\times] a_{3}, b_{3}[)=: I_{p}\right.
$$

A $i-$ th $(i \in\{1,2,3\})$ coordinate curve is obtained fixing in the function $\varphi$ all arguments except the $x_{i}$ variable, and the tangent vectors of such coordinate curves are denoted as follows

$$
\left(\forall q \in I_{p}\right)\left(a_{i}(q):=\frac{\partial \varphi}{\partial x_{i}}\left(\varphi^{-1}(q)\right)\right) .
$$

Obviously for every $q \in I_{p}$ the set $\left\{a_{i}(q), i \in\{1,2,3\}\right\}$ is a basis of the vector space of translations in $E^{3}$. Therefore the curvilinear coordinate system which has been introduced generates a field of bases in all $I_{p}$.

Definition 5.10. In $I_{p}$ we can introduce the following scalar fields

$$
\left(\forall q \in I_{p}\right)\left(g_{i j}(q):=a_{i}(q) \cdot a_{j}(q)\right)
$$

which are the components, in considered curvilinear coordinate system, of the Riemann metric in the Euclidean field $E^{3}$.

Let us consider a piecewise regular surface: as we will see in what follows, it is possible to introduce in the neighborhood of $p$ charts (i.e. curvilinear coordinate systems) in the Euclidean space $E^{3}$ which are "adapted" to i) the surface in the neighborhood of regular point $p \in S-(\partial S \cup \partial \partial S)$ ii) to the edge of the surface in the neighborhood of a point which is not a wedge.

\section{Local curvilinear coordinate system in $E^{3}$ adapted to $S$ in the neighborhood of a regular point.}

The following Lemma is a consequence of the inverse function Theorem and is the basis of an important part of Gaussian differential geometry (for a proof see e.g. Kosinski (1986), dell'Isola and Kosinski (1989).

Lemma 5.11. Let $r$ be a local parametrization of $S$ in the neighborhood of the regular point $p \in S-(\partial S \cup \partial \partial S)$. For a suitably small positive $\varepsilon$ the map $\varphi$ defined by

$$
\begin{aligned}
\varphi & \left.:\left(x^{1}, x^{2}, x^{3}\right) \in\right] a_{1}, b_{1}[\times] a_{2}, b_{2}[\times]-\varepsilon,+\varepsilon\left[\subset \mathbb{R}^{3}\right. \\
& \mapsto r\left(x^{1}, x^{2}\right)+x^{3} N\left(r^{-1}\left(x^{1}, x^{2}\right)\right) \in E^{3}
\end{aligned}
$$

actually is an invertible function and a diffeomorphism. 
Definition 5.12. When the map $\varphi$ considered in the previous Lemma is a diffeomorphism it is the called the chart adapted to $S$ induced by the parametrization $r$.

When this will not be cause of confusion we will admit an abuse of notation and we will use the symbols $N\left(x^{1}, x^{2}\right), g_{\alpha \beta}\left(x^{1}, x^{2}\right)$ and $a_{\alpha}\left(x^{1}, x^{2}\right)$ instead of the symbols $N\left(r^{-1}\left(x^{1}, x^{2}\right)\right), g_{\alpha \beta}\left(r^{-1}\left(x^{1}, x^{2}\right)\right)$ and $a_{\alpha}\left(r^{-1}\left(x^{1}, x^{2}\right)\right)$. The same abuse of notation will be repeated for all the fields $a_{i}$ and $g_{i j}$ not specifying the composition with the function $\varphi^{-1}$.

The class of charts we have now introduced was first introduced by Gauss (see e.g. Spivak (1979)).

We are now able to extend in the neighborhood in $E^{3}$ of a regular point $p \in S$ the fields of projectors in the tangent and in the orthogonal bundles:

Definition 5.13. Let us consider a chart $\varphi$ adapted to the surface $S$ in the neighborhood of a point $p$. For every $\left.x_{3} \in\right]-\varepsilon, \varepsilon[$ we can consider the (regular) surface $S_{x_{3}}$ which is defined by the following local parametrization

$$
r_{x_{3}}:=r+x_{3} N
$$

On every surface $S_{x_{3}}$ it is easy to introduce the tangent and orthogonal projectors, which for $x_{3}=0$ reduce to the projectors introduced already for $S=S_{0}$. We will denote these fields of projectors, also when defined in the opens set

$$
\varphi(] a_{1}, b_{1}[\times] a_{2}, b_{2}[\times]-\varepsilon,+\varepsilon[) \subset E^{3}
$$

with the same symbols $P_{S, p}$ and $Q_{S, p}$.

\section{Local curvilinear coordinate system in $E^{3}$ adapted to an edge of $S$ in the neighborhood of a point which is not a wedge.}

Let $r$ be a local parametrization of an edge $\gamma$ of $S$ in the neighborhood of a point $p \in \partial S-\partial \partial S$. When it is a diffeomorphism, we will call the map $\varphi$ defined by

$$
\begin{aligned}
\varphi & \left.:\left(x^{1}, x^{2}, x^{3}\right) \in\right] a_{1}, b_{1}[\times]-\varepsilon,+\varepsilon[\times]-\varepsilon,+\varepsilon\left[\subset \mathbb{R}^{3}\right. \\
& \mapsto r\left(x^{1}\right)+x^{2} N^{ \pm}\left(r^{-1}\left(x^{1}\right)\right)+x^{3} \nu^{ \pm}\left(r^{-1}\left(x^{1}\right)\right) \in E^{3}
\end{aligned}
$$

a chart adapted to $S$ at the considered edge as induced by the edge parametrization $r$ on the side + or - (respectively) depending on the consistent choice adopted. 
Adapting the argument used in the proof of the Lemma of the previous subsection, and using the assumed regularity hypotheses about $S$ and its edges, it can be proven again that, when the value of $\varepsilon$ is chosen to be positive and suitably small, $\varphi$ actually is a diffeomorphism and can be used as a chart in $E^{3}$.

Definition 5.14. Let us consider a regular point of the edge $\gamma$ and a chart adapted to $S$ at $\gamma$. For every $\left.\left(x^{2}, x^{3}\right) \in\right]-\varepsilon,+\varepsilon[\times]-\varepsilon,+\varepsilon[$ we can consider the regular curves parametrized by the function

$$
r_{x^{2}, x^{3}}^{ \pm}:=r+x^{2} N^{ \pm}+x^{3} \nu^{ \pm}
$$

For each of these curves we can define the projection on the orthogonal and tangent bundles, thus obviously extending in the neighborhood of $\gamma$ the already introduced projection fields $P_{\gamma, p}$ and $Q_{\gamma, p}$.

\section{Gauss Divergence Theorem for embedded Riemannian manifolds}

We choose a global othonormal basis $\left(e_{i}, i=1,2,3\right)$ for the vector field of displacements in $E^{3}$. All tensor fields, unless differently specified, will be represented by means of the components with respect this basis. In this section we consider an embedded Riemannian manifold $M$ in $E^{3}$. This manifold can be therefore a regular curve or surface. Because we were able to construct the so-called Gaussian coordinate systems adapted to considered manifolds, then in a whole neighborhood of these manifolds it is possible to introduce the projection operator fields $P$ and $Q$. For reducing the complication of the calculation which we will perform in what follows we do not use directly the adapted curvilinear coordinates: instead, after having established the existence of the fields $P$ and $Q$ in the neighborhood of $M$,we introduce a global Cartesian coordinate system and represent all fields in it. This technical choice is exactly the same one which allowed to Germain the generalization, for second gradient materials, of the results found by Green, Rivlin, Toupin and Mindlin.

It is easy to prove the following:

Lemma 6.1. If on each manifold $M, P$ denotes the projection on the tangent bundle : we have

$$
\begin{gathered}
\delta_{i}^{j}=P_{i}^{j}+Q_{i}^{j}, \quad P_{i}^{j} P_{j}^{k}=P_{i}^{k}, \\
Q_{i}^{j} Q_{j}^{k}=Q_{i}^{k}, \quad P_{i}^{j} Q_{j}^{k}=0 .
\end{gathered}
$$


The unit external normal to $M$ on its border is denoted $\nu$; it belongs to the tangent space to $M$.

Using these notations the divergence theorem reads (see e.g. Spivak (1979))

Theorem 6.2. For any vector field $W$ defined in the vicinity of $M$

$$
\int_{M}\left(P_{j}^{i} W^{j}\right)_{, k} P_{i}^{k}=\int_{\partial M} W^{i} P_{i}^{k} \nu_{k}
$$

This theorem together with relation

$$
Q_{j, k}^{i} P_{i}^{k}=-Q_{j}^{i} P_{i, k}^{k}
$$

implies that

Corollary 6.3. For any vector field $W$ defined in a neighborhood of $M$

$$
\begin{gathered}
\int_{M}\left(W^{i}\right)_{, k} P_{i}^{k}=\int_{M}\left(P_{j}^{i} W^{j}\right)_{, k} P_{i}^{k}+\left(Q_{j}^{i} W^{j}\right)_{, k} P_{i}^{k}= \\
=\int_{M} W^{j} Q_{j, k}^{i} P_{i}^{k}+\int_{\partial M} W^{i} P_{i}^{k} \nu_{k}= \\
=-\int_{M} W^{j} Q_{j}^{i} P_{i, k}^{k}+\int_{\partial M} W^{i} P_{i}^{k} \nu_{k} .
\end{gathered}
$$

\section{Power expended by internal or external actions}

Once we fix a subbody $B$ of a given continuous body $C$ and consider the set $\mathcal{A}$ of all admissible velocity fields for $B$ it is natural to admit that in $\mathcal{A}$ are included all "test functions" (i.e. infinitely differentiable functions) having compact support.

It is also natural (as done e.g. by Salençon (1988-2005) or Germain (1973)) to assume that the power expended by internal or external actions (with respect to $B$ ) is a linear and continuous functional when defined in the set of test functions (with respect to Frechèt topology).

In other word we accept the following

\section{Postulate (Power)}

The power expended by actions exerted on or in a subbody $B$ is a distribution (in the sense of Schwartz). 
It is clear that, once the previous postulate is accepted, the following Theorems and Definitions, due to Schwartz (1973), become really relevant in continuum mechanics:

Theorem 7.1. Every distribution having compact support $K$ can be, in infinitely equivalent ways, represented as the sum of a finite number of derivatives (in the weak sense) of continuous functions all having their support included in a neighborhood of $K$.

Definition 7.2. A distribution is said to have order smaller than or equal to $k$ if one can represent it as the sum of derivatives of continuous functions all having order smaller than or equal to $k$.

Theorem 7.3. Every distribution having support included in a regular embedded submanifold $M$ can be uniquely decomposed as a finite sum of transversal derivatives of extensions of distributions defined on $M$.

7.1 Representations of distributions which are $N-t h$ order derivatives of absolutely continuous measures concentrated on submanifolds of $R^{H}$ with border

In what follows we will need to consider distributions having support concentrated on a regular submanifold $M$ embedded in $R^{H}$ which are exactly the $N-t h$ order derivatives of measures which are absolutely continuous with respect to the corresponding Hausdorff measure.

More specifically we will be interested to consider distributions having the form

$$
\mathcal{P}(V)=\int_{M} T \cdot \nabla^{N} V
$$

where $T$ is a suitably integrable (with respect to the Hausdorff measure of M) $N$-times contravariant tensor field.

We have now to get a Lemma which is essential when one wants to study the structure of contact actions in $n-t h$ order continua.

Lemma 7.4. Let us consider a Riemannian "regular" embedded manifold $M$. Let $T$ be a symmetric tensor field defined in the neighborhood of $M$. Let us introduce the following "projected" field

$$
\mathbb{P}(T)^{j_{1} \ldots j_{N-1} l}=\left(\sum_{\alpha=0}^{N-1} C_{\alpha}^{N} T^{i_{1} i_{2} \ldots i_{N}} Q_{i_{1}}^{j_{1}} . . Q_{i_{\alpha}}^{j_{\alpha}} P_{i_{\alpha+1}}^{j_{\alpha+1}} . . P_{i_{N-1}}^{j_{N-1}}\right) P_{i_{N}}^{l}
$$


where $C_{\alpha}^{N}$ denotes the appropriate binomial coefficient. Then the following formula of integration by parts holds:

$$
\begin{gathered}
\int_{M} T^{i_{1} i_{2} \ldots i_{N}} V,,_{i_{1} i_{2} \ldots i_{N}}= \\
=\int_{M} T^{i_{1} i_{2} \ldots i_{N}} Q_{i_{1}}^{j_{1}} Q_{i_{2}}^{j_{2}} . . Q_{i_{N}}^{j_{N}} V, j_{1} j_{2} \ldots j_{N}+ \\
-\int_{M}\left(\left(\mathbb{P}(T)^{j_{1} \ldots j_{N-1} l}\right),_{j_{N}} P_{l}^{j_{N}}\right) V,,_{j_{1} j_{2} \ldots j_{N-1}}+ \\
+\int_{\partial M} \mathbb{P}(T)^{j_{1} \ldots j_{N-1} l} V,,_{j_{1} j_{2} \ldots j_{N-1}} P_{l}^{j_{N}} \nu_{j_{N}}
\end{gathered}
$$

Proof. We start decomposing identity tensor field in terms of tangent and orthogonal projector fields adapted to $M$

$$
\begin{aligned}
& \int_{M} T^{i_{1} i_{2} \ldots i_{N}} V, i_{1} i_{2} \ldots i_{N}= \\
& =\int_{M} T^{i_{1} i_{2} \ldots i_{N}} V,{j_{1} j_{2} \ldots j_{N}}\left(P_{i_{1}}^{j_{1}}+Q_{i_{1}}^{j_{1}}\right)\left(P_{i_{2}}^{j_{2}}+Q_{i_{2}}^{j_{2}}\right) \ldots\left(P_{i_{N}}^{j_{N}}+Q_{i_{N}}^{j_{N}}\right) .
\end{aligned}
$$

Subsequently we use the symmetry of $T$ and orthogonality of $P$ and $Q$ projectors thus obtaining with simple algebra

$$
\begin{aligned}
& \int_{M} T^{i_{1} i_{2} \ldots i_{N}} V, i_{1} i_{2} \ldots i_{N}= \\
& =\int_{M} \sum_{\alpha=0}^{N} C_{\alpha}^{N}\left(T^{i_{1} i_{2} \ldots i_{N}} Q_{i_{1}}^{j_{1}} . . Q_{i_{\alpha}}^{j_{\alpha}} V, j_{1} j_{2} \ldots j_{N} P_{i_{\alpha+1}}^{j_{\alpha+1}} . . P_{i_{N}}^{j_{N}}\right)= \\
& =\int_{M} T^{i_{1} i_{2} \ldots i_{N}} Q_{i_{1}}^{j_{1}} Q_{i_{2}}^{j_{2}} . . Q_{i_{N}}^{j_{N}} V{, j_{1} j_{2} \ldots j_{N}}+ \\
& +\int_{M} \sum_{\alpha=0}^{N-1} C_{\alpha}^{N}\left(T^{i_{1} i_{2} \ldots i_{N}} Q_{i_{1}}^{j_{1}} . . Q_{i_{\alpha}}^{j_{\alpha}} V,_{j_{1} j_{2} \ldots j_{N}} P_{i_{\alpha+1}}^{j_{\alpha+1}} . . P_{i_{N}}^{j_{N}}\right) .= \\
& =\int_{M} T^{i_{1} i_{2} \ldots i_{N}} Q_{i_{1}}^{j_{1}} Q_{i_{2}}^{j_{2}} . . Q_{i_{N}}^{j_{N}} V, j_{1} j_{2} \ldots j_{N}+ \\
& +\int_{M}\left(\sum_{\alpha=0}^{N-1} C_{\alpha}^{N} T^{i_{1} i_{2} \ldots i_{N}} Q_{i_{1}}^{j_{1}} . . Q_{i_{\alpha}}^{j_{\alpha}} P_{i_{\alpha+1}}^{j_{\alpha+1}} . . P_{i_{N-1}}^{j_{N-1}}\right) V,_{j_{1} j_{2} \ldots j_{N-1} j_{N}} P_{i_{N}}^{j_{N}} .
\end{aligned}
$$

We finally introduce the definition of the projected field $\mathbb{P}(T)$, use Leibnitz 
differentiation rule and apply Divergence Theorem on $M$

$$
\begin{aligned}
& \int_{M} T^{i_{1} i_{2} \ldots i_{N}} V, i_{1} i_{2} \ldots i_{N}= \\
& =\int_{M} T^{i_{1} i_{2} \ldots i_{N}} Q_{i_{1}}^{j_{1}} Q_{i_{2}}^{j_{2}} . . Q_{i_{N}}^{j_{N}} V, j_{1} j_{2} \ldots j_{N}+ \\
& +\int_{M} \mathbb{P}(T)^{j_{1} \ldots j_{N-1} l} V, j_{1 j_{2} \ldots j_{N-1} j_{N}} P_{l}^{j_{N}}= \\
& =\int_{M} T^{i_{1} i_{2} \ldots i_{N}} Q_{i_{1}}^{j_{1}} Q_{i_{2}}^{j_{2}} . . Q_{i_{N}}^{j_{N}} V, j_{1} j_{2} \ldots j_{N}+ \\
& -\int_{M}\left(\mathbb{P}(T)^{j_{1} \ldots j_{N-1} l}\right), j_{N} P_{l}^{j_{N}} V{, j_{1} j_{2} \ldots j_{N-1}}+ \\
& +\int_{M}\left(\mathbb{P}(T)^{j_{1} \ldots j_{N-1} l} V,{, j_{1} j_{2} \ldots j_{N-1}}\right){, j_{N}}_{l} P_{l}^{j_{N}}= \\
& =\int_{M} T^{i_{1} i_{2} \ldots i_{N}} Q_{i_{1}}^{j_{1}} Q_{i_{2}}^{j_{2} . .} Q_{i_{N}}^{j_{N}} V, j_{1} j_{2} \ldots j_{N}+ \\
& -\int_{M}\left(\left(\mathbb{P}(T)^{j_{1} \ldots j_{N-1} l}\right),,_{N} P_{l}^{j_{N}}\right) V,,_{j_{1} j_{2} \ldots j_{N-1}}+ \\
& +\int_{\partial M} \mathbb{P}(T)^{j_{1} \ldots j_{N-1} l} V_{j_{1} j_{2} \ldots j_{N-1}} P_{l}^{j_{N}} \nu_{j_{N}}
\end{aligned}
$$

so concluding the proof.

Remark 7.5. If $T$ is a $N-t h$ order tensor field then also $\mathbb{P}(T)$ is a $N-t h$ order tensor field.

Definition 7.6. Let $S$ be a $N-$ th order tensor field having $S^{i_{1} i_{2} \ldots i_{N}}$ as component fields. We denote by $\operatorname{div}_{M} S$ the tensor field having the components $\left(\left(S^{j_{1} \ldots j_{N-1} l}\right), j_{N} P_{l}^{j_{N}}\right)$. Obviously $\operatorname{div}_{M} S$ is a tensor field of order $N-1$.

Definition 7.7. We will denote $S_{\perp}$ the tensor field (equally of order $N$ ) having the following components $S^{i_{1} i_{2} \ldots i_{N}} Q_{i_{1}}^{j_{1}} Q_{i_{2}}^{j_{2}} . . Q_{i_{N}}^{j_{N}}$.

Using the notation introduced in the previous remark and denoting with a dot the complete saturation of contravariant with covariant indices, the 
formula obtained in the previous Lemma reads as follows

$$
\begin{gathered}
\int_{M} T \cdot \nabla^{N} V=\int_{M} T_{\perp} \cdot\left(\nabla^{N} V\right)_{\perp}+ \\
-\int_{M}\left(\operatorname{div}_{M} \mathbb{P}(T)\right) \cdot \nabla^{N-1} V+ \\
\quad+\int_{\partial M} \mathbb{P}(T) \cdot\left(\nabla^{N-1} V \otimes \nu\right)
\end{gathered}
$$

We can now apply a second time the Lemma thus obtaining

$$
\begin{aligned}
& \int_{M} T \cdot \nabla^{N} V= \int_{M} T_{\perp} \cdot\left(\nabla^{N} V\right)_{\perp}+\int_{\partial M} \mathbb{P}(T) \cdot\left(\nabla^{N-1} V \otimes \nu\right) \\
& \quad-\int_{M}\left(\operatorname{div}_{M} \mathbb{P}(T)\right) \cdot \nabla^{N-1} V= \\
&=\left(\int_{M} T_{\perp} \cdot\left(\nabla^{N} V\right)_{\perp}\right)+\int_{\partial M} \mathbb{P}(T) \cdot\left(\nabla^{N-1} V \otimes \nu\right)+ \\
&-\left(\int_{M}\left(\operatorname{div}_{M} \mathbb{P}(T)\right)_{\perp} \cdot\left(\nabla^{N-1} V\right)_{\perp}\right)+ \\
&+\left(\int_{M} \operatorname{div}_{M}\left(\mathbb{P}\left(\operatorname{div}_{M} \mathbb{P}(T)\right)\right) \cdot \nabla^{N-2} V\right)+ \\
&-\int_{\partial M} \mathbb{P}\left(\operatorname{div}_{M} \mathbb{P}(T)\right) \cdot\left(\nabla^{N-2} V \otimes \nu\right) .
\end{aligned}
$$

Applying exactly $N$ times the Lemma we get the following

Corollary 7.8. Under the same assumptions of the previous lemma and having introduced the conventions

$$
\left(\operatorname{div}_{M} \mathbb{P}\right)^{0} T=T, \quad\left(\operatorname{div}_{M} \mathbb{P}\right)^{\alpha} T=\underbrace{\operatorname{div}_{M} \mathbb{P}\left(\operatorname{div}_{M} \mathbb{P} \ldots \ldots\left(\operatorname{div}_{M} \mathbb{P}(T)\right)\right.}_{\alpha \text { times }}
$$

the following equality holds if $T$ is a symmetric tensor field $K$ - times contravariant :

$$
\begin{aligned}
\int_{M} T \cdot \nabla^{K} V= & \sum_{\alpha=0}^{K-1}(-1)^{\alpha} \int_{M}\left(\left(\operatorname{div}_{M} \mathbb{P}\right)^{\alpha} T\right)_{\perp} \cdot\left(\nabla^{K-\alpha} V\right)_{\perp}+ \\
& +(-1)^{K} \int_{M}\left(\left(\operatorname{div}_{M} \mathbb{P}\right)^{K} T\right) V+ \\
& +\sum_{\alpha=0}^{K-1}(-1)^{\alpha} \int_{\partial M} \mathbb{P}\left(\left(\operatorname{div}_{M} \mathbb{P}\right)^{\alpha} T\right) \cdot\left(\nabla^{K-1-\alpha} V \otimes \nu\right)
\end{aligned}
$$


An alternative expression for the previous representation formula is (the lower dot indicates the saturation of the last index of the tensor at its left with the first index of the tensor at his right) obtained by renominating the indices:

$$
\begin{aligned}
\int_{M} T \cdot \nabla^{K} V=\sum_{J=1}^{K}(-1)^{K-J} \int_{M}\left(\left(\operatorname{div}_{M} \mathbb{P}\right)^{K-J} T\right)_{\perp} \cdot\left(\nabla^{J} V\right)_{\perp}+ \\
+(-1)^{K} \int_{M}\left(\left(\operatorname{div}_{M} \mathbb{P}\right)^{K} T\right) V+ \\
+\sum_{L=0}^{K-1}(-1)^{K-1-L} \int_{\partial M} \mathbb{P}\left(\left(\operatorname{div}_{M} \mathbb{P}\right)^{K-1-L} T\right) \cdot \nu \cdot \nabla^{L} V
\end{aligned}
$$

The previous corollary allows us to find the unique representation in terms of transversal derivatives of the distribution of the type specified by equation 17. The existence of this representation is stated in Theorem 7.3.

\section{Principle of Virtual Powers applied to $N-t h$ gradient continua.}

On a continuous body $B$ external world can exert actions. While deformation processes occur in the same body its subbodies interact because of internal actions. We call "internal" the power expended on an admissible velocity by internal actions, and "external" the power expended on an admissible velocity by external actions.

The following definitions are clearly inspired by Schwartz representation theorem: however it has to be remarked that such definitions were put forward by Green and Rivlin who, most likely, were unaware of Schwartz results.

Definition 8.1. We call $N-t h$ gradient continuum a continuous body $B$ for which the internal power $\mathcal{P}^{\text {int }}$ is a distribution of order smaller or equal to $N$. In formulas (the dot representing the saturation of contravariant with covariant indices)

$$
\mathcal{P}^{\text {int }}(B, V)=\sum_{\Lambda=0}^{N} \int_{B} T_{\Lambda} \cdot \nabla^{\Lambda} V .
$$

Following Green and Rivlin we will call the $\Lambda$ - times contravariant tensors $T_{\Lambda}$ " $\Lambda$-th order multipolar stress".

Remark 8.2. The previous definition is clearly a "constitutive" assumption, specifying the "type" of internal actions which are considered "possi- 
ble" inside the body. The Theorem 7.1, once one accepts the Postulate 7, proves that for a given body the internal power must be of finite order.

The energy transfer per unit time from the external world to the body $B$ can also be calculated by means of a suitable distribution calculated on the admissible velocity field $V$.

We assume that in order to distinguish long range actions from contact actions the power of external actions $\mathcal{P}^{\text {ext }}$ exerted on the body $B$ must be represented by means of the theorem 7.3: long range external actions exerted on $B$ can be represented a distribution which is a integrable function with respect threedimensional Lebesgue measure, while contact actions are concentrated on the contact surface of $B$ and are distributions which can be transverse distributional derivatives on the regular part of this surface and on its edges and wedges: these distributions will be represented by means of integrals calculated with Hausdorff measures of dimension two or one or by means of sums of Dirac Deltas.

We are now ready to add a second (for a beautiful presentation of the ideas inspiring this axiom the reader is referred to Salençon(1988) or to Cosserat (1908-1909))

\section{Postulate (Principle of Virtual Powers or Power Balance)}

For every subbody $S$ of a given body and for every test velocity field $V$ the following equality holds

$$
\mathcal{P}^{i n t}(B, V)=\mathcal{P}^{e x t}(B, V) .
$$

\section{Contact actions in $N-$ th order strain-gradient multipolar continua: $N-$ th order forces and stress multipoles.}

The Principle of Virtual Powers clearly implies that the admissible external contact actions which a $N-t h$ gradient continuum can "sustain" belong to a particular subset of the set of distributions concentrated on $\partial B, \partial \partial B$ and $\partial \partial \partial B$. The argument we develop here follows the same spirit as the papers cited in the references by Green, Rivlin and Germain. Also of relevance are the considerations in Seppecher (1987)-(1989).

In the following we want specify the aforementioned class. 
Lemma 9.1. Let $B$ be a $N$ - th gradient continuum. Let us assume that the topological boundary of $B$ is a piecewise regular surface as defined in 5.1. If the equality 56 holds then for the part of $\mathcal{P}^{e x t}$ having support on $\partial B, \partial \partial B$ and $\partial \partial \partial B$ (i.e. for the external actions which are contact actions concentrated on the topological boundary of $B$ ) the following properties hold i) the part of $\mathcal{P}^{\text {ext }}$ having support on $\partial B$ and absolutely continuous with respect to $H^{2}$ measure is a distribution of order smaller or equal to $N-1$, ii) the part of $\mathcal{P}^{\text {ext }}$ having support on $\partial \partial B$ and absolutely continuous with respect to $H^{1}$ measure is a distribution of order smaller or equal to $N-2$, iii) the part of $\mathcal{P}^{\text {ext }}$ having support on $\partial \partial \partial B$ is a distribution concentrated on points, constituted by derivatives of Dirac Deltas of order smaller or equal to $N-2$.

Proof. The proof is easily obtained repeatedly applying to the addends of 55 the Corollary 7.8 to the embedded Riemannian manifolds which constitutes the regular parts of the topological boundary of $B$ and its edges.

Because of the previous Lemma and the Corollary 7.8 the following representation form for $\mathcal{P}^{e x t}$ holds

Lemma 9.2. The external actions which can be sustained by a $N$ - th gradient continuum can only expend powers of the type

$$
\begin{aligned}
\mathcal{P}^{e x t}(B, V) & =\int_{\mathcal{B}} E V+\int_{\mathcal{S}_{B}} F_{0} V+\sum_{\Delta=1}^{N-1} \int_{\mathcal{S}_{B}} F_{\Delta} \cdot\left(\nabla^{\Delta} V\right)_{\perp}+ \\
& +\int_{\mathcal{E}_{B}} G_{0} \cdot V+\sum_{\Delta=1}^{N-2} \int_{\mathcal{E}_{B}} G_{\Delta} \cdot\left(\nabla^{\Delta} V\right)_{\perp}+\sum_{\Delta=0}^{N-3} \int_{\mathcal{W}_{B}} H_{\Delta} \cdot \nabla^{\Delta} V
\end{aligned}
$$

where we used the notations $\mathcal{S}_{B}:=\operatorname{Support}(\partial B), \mathcal{E}_{B}:=\operatorname{Support}(\partial \partial B)$ and $\mathcal{W}_{B}:=\operatorname{Support}(\partial \partial B)$

Remark 9.3. It is needed here (for more details see e.g. Arnold (1979)) to precise the relationship between e.g. $\partial \partial B$ and its support. If an edge is the border of two different regular surfaces belonging to $\partial B$ then $\partial \partial B$ is the union of the two borders of these concurring surfaces, with a sign depending on the relative orientation of the curve, which constitutes the support of the two different borders, and the concurring surface. These definitions are those used generally in the theory of integration of differential forms defined on differential manifolds. 
Definition 9.4. The fields $F_{\Delta}, G_{\Delta}$ and $H_{\Delta}$ are called by Green and Rivlin "contact $n$ - forces" per unit surface, per unit line or concentrated on points.

Remark 9.5. In all previous considerations we have used the following obvious but important facts: i) when applying Corollary 7.8 to the body $B$ there is no transverse direction in the embedding, ii) when the same corollary was applied to regular parts of the topological boundary of $B$ and to its edges then the transverse directions in the corresponding embeddings are the normal to the surfaces or the planes orthogonal to the tangents to the edges, iii) in wedges all directions are transverse.

We want now to find how to generalize Cauchy representation Theorem for contact force densities in terms of Cauchy stress to multipolar $N$ - th gradient continua basing it again on the Postulate 8 and on the Corollary 7.8 .

We start from the formula 55

$$
\mathcal{P}^{\text {int }}(B, V)=\sum_{\Lambda=0}^{N} \int_{B} T_{\Lambda} \cdot \nabla^{\Lambda} V .
$$

and apply to each of its addends the formula obtained in the Corollary 7.8 when first identifying $M$ with $B$, the projection operator $\mathbb{P}$ with the identity and the outer normal $\nu$ with the normal $n$ to the surface $\partial B$, we get

$$
\begin{gathered}
\mathcal{P}^{i n t}(B, V)=\sum_{\Lambda=0}^{N} \int_{B} T_{\Lambda} \cdot \nabla^{\Lambda} V= \\
=\int_{B}\left(\sum_{\Lambda=0}^{N}(-1)^{\Lambda} d i v^{\Lambda} T_{\Lambda}\right) V+ \\
+\sum_{\Lambda=0}^{N} \sum_{L=0}^{\Lambda-1} \int_{\partial B}\left((-1)^{\Lambda-1-L}(d i v)^{\Lambda-1-L} T_{\Lambda}\right) \cdot n \cdot \nabla^{L} V
\end{gathered}
$$

We now simply manipulate, using associativity of summation, the obtained expression for getting the coefficients of every power of $\nabla V$ appearing in it. Therefore the equality

$$
\mathcal{P}^{\text {int }}(B, V)=\sum_{\Lambda=0}^{N} \int_{B} T_{\Lambda} \cdot \nabla^{\Lambda} V
$$

becomes 


$$
\begin{gathered}
\mathcal{P}^{i n t}(B, V)=\int_{B}\left(\sum_{\Lambda=0}^{N}(-1)^{\Lambda} \operatorname{div}^{\Lambda} T_{\Lambda}\right) V+ \\
\sum_{L=0}^{N-1} \int_{\partial B}\left(\sum_{\Lambda=L+1}^{N}(-1)^{\Lambda-1-L} \operatorname{div}^{\Lambda-1-L} T_{\Lambda} \cdot n\right) \cdot \nabla^{L} V
\end{gathered}
$$

A simple inspection of equations 63 and 65 makes natural the introduction of to the following

\section{Definition 9.6. Bulk and Surface Stress Tensors}

$$
T(B, \Lambda):=T_{\Lambda} ; \quad T(\partial B, L):=\left(\sum_{\Lambda=L+1}^{N}(-1)^{\Lambda-1-L} d i v^{\Lambda-1-L} T_{\Lambda} \cdot n\right)
$$

\section{Definition 9.7. Bulk Internal Force}

$$
F(B, 0):=\left(\sum_{\Lambda=0}^{N}(-1)^{\Lambda} \operatorname{div}^{\Lambda}(T(B, \Lambda))\right)
$$

By means of introduced notations the expression of internal power is meaningfully simplified as follows:

$$
\mathcal{P}^{i n t}(B, V)=\int_{B} F(B, 0) V+\sum_{L=0}^{N-1} \int_{\partial B} T(\partial B, L) \cdot \nabla^{L} V
$$

We now apply the Corollary 7.8 , by identifying the embedded manifold $M$ with any of the regular parts of $\partial B$ to transform the integrals involving surface stresses thus obtaining:(in the following formulas $\nu$ represents the unit normal to $\partial \partial B$ which is tangent to $\partial B$ and we denoted with the symbol $\mathbb{P}_{\sigma}$ the projector operator relative to the tangent planes of every the regular parts of $\partial B$ )

$$
\begin{gathered}
\int_{\partial B} T(\partial B, L) \cdot \nabla^{L} V= \\
=\sum_{J=1}^{L}(-1)^{L-J} \int_{\partial B}\left(\left(\operatorname{div}_{\partial B} \mathbb{P}_{\sigma}\right)^{L-J} T(\partial B, L)\right)_{\perp} \cdot\left(\nabla^{J} V\right)_{\perp}+ \\
+(-1)^{L} \int_{\partial B}\left(\left(d i v_{\partial B} \mathbb{P}_{\sigma}\right)^{L} T(\partial B, L)\right) V+ \\
+\sum_{J=0}^{L-1}(-1)^{L-1-J} \int_{\partial \partial B} \mathbb{P}_{\sigma}\left(\left(\operatorname{div}_{\partial B} \mathbb{P}_{\sigma}\right)^{L-1-J} T(\partial B, L)\right) . \nu \cdot \nabla^{J} V
\end{gathered}
$$


We get for the sum of all terms involving surface stresses

$$
\begin{gathered}
\sum_{L=0}^{N-1} \int_{\partial B} T(\partial B, L) \cdot \nabla^{L} V= \\
=\sum_{L=0}^{N-1} \sum_{J=1}^{L}(-1)^{L-J} \int_{\partial B}\left(\left(\operatorname{div}_{\partial B} \mathbb{P}_{\sigma}\right)^{L-J} T(\partial B, L)\right)_{\perp} \cdot\left(\nabla^{J} V\right)_{\perp}+ \\
+\sum_{L=0}^{N-1}(-1)^{L} \int_{\partial B}\left(\left(\operatorname{div}_{\partial B} \mathbb{P}_{\sigma}\right)^{L} T(\partial B, L)\right) V \\
+\sum_{L=0}^{N-1} \sum_{J=0}^{L-1}(-1)^{L-1-J} \int_{\partial \partial B} \mathbb{P}_{\sigma}\left(\left(\operatorname{div}_{\partial B} \mathbb{P}_{\sigma}\right)^{L-1-J} T(\partial B, L)\right) \cdot \nu \cdot \nabla^{J} V
\end{gathered}
$$

Using again associativity of summation the last equality becomes

$$
\begin{gathered}
\sum_{L=0}^{N-1} \int_{\partial B} T(\partial B, L) \cdot \nabla^{L} V= \\
=\sum_{J=1}^{N-1} \int_{\partial B}\left(\sum_{L=J}^{N-1}(-1)^{L-J}\left(\operatorname{div}_{\partial B} \mathbb{P}_{\sigma}\right)^{L-J} T(\partial B, L)\right)_{\perp} \cdot\left(\nabla^{J} V\right)_{\perp}+\quad(74) \\
+\int_{\partial B}\left(\sum_{L=0}^{N-1}(-1)^{L}\left(\operatorname{div}_{\partial B} \mathbb{P}_{\sigma}\right)^{L} T(\partial B, L)\right) V \\
+\sum_{J=0}^{N-2} \int_{\partial \partial B}\left(\sum_{L=J+1}^{N-1}(-1)^{L-1-J} \mathbb{P}_{\sigma}\left(\left(\operatorname{div}_{\partial B} \mathbb{P}_{\sigma}\right)^{L-1-J} T(\partial B, L)\right) \cdot \nu\right) \cdot \nabla^{J} V
\end{gathered}
$$

The nomenclature introduced in the previous definitions (which are based on those introduced by Green and Rivlin) allows us a meaningful interpretation of obtained result

\subsection{The terms of surface (1-)forces and surface $L+1$ - forces}

Surface density $F(\partial B, 0)$ of $(1-)$ force appears in the addend 75

$$
F(\partial B, 0):=\sum_{L=0}^{N-1}(-1)^{L}\left(\operatorname{div}_{\partial B} \mathbb{P}_{\sigma}\right)^{L} T(\partial B, L)
$$

The introduced vector quantity $F(\partial B, 0)$ generalizes Cauchy "traction" vector: it is expending power on (virtual) velocities. 
Remark 9.8. In Cauchy Continua surface density of force $F(\partial B, 0)$ coincides with the only non-vanishing surface stress $T(\partial B, 0)$. In Second Gradient Continua (as established by Green and Rivlin, Mindlin and Germain) the following equality holds

$$
F(\partial B, 0)=T(\partial B, 0)-\left(\operatorname{div}_{\partial B} \mathbb{P}_{\sigma}\right) T(\partial B, 1)
$$

We then consider the addend 74 which leads us to the definition

$$
F(\partial B, J):=\left(\sum_{L=J}^{N-1}(-1)^{L-J}\left(\operatorname{div}_{\partial B} \mathbb{P}_{\sigma}\right)^{L-J} T(\partial B, L)\right)_{\perp}
$$

Surface $J+1-$ forces $F(\partial B, J)$ expend power on the $J-t h$ transverse gradient of (virtual) velocity fields.

Finally we consider the addend 76 which leads us naturally to the following

Definition 9.9. Line Stress Tensors

$$
T(\partial \partial B, L):=\cdot\left(\sum_{L=J+1}^{N-1}(-1)^{L-1-J} \mathbb{P}_{\sigma}\left(\left(\operatorname{div}_{\partial B} \mathbb{P}_{\sigma}\right)^{L-1-J} T(\partial B, L)\right) . \nu\right)
$$

We have thus proven the following intermediate:

Lemma 9.10. For $N$ - th gradient continua the following representation formula holds

$$
\begin{gathered}
\mathcal{P}^{i n t}(B, V)=\int_{B} F(B, 0) V+\int_{\partial B} F(\partial B, 0) \cdot V \\
+\sum_{L=1}^{N-1} \int_{\partial B} F(\partial B, L) \cdot\left(\nabla^{L} V\right)_{\perp}+\sum_{L=0}^{N-2} \int_{\partial \partial B} T(\partial \partial B, L) \cdot \nabla^{L} V
\end{gathered}
$$

We must now proceed by applying again the Corollary 7.8 (when this time $M$ is any regular curve with border belonging to $\partial \partial B$ ) to the terms involving line stresses. Once we have introduced the projection operator $\mathbb{P}_{\lambda}$ relative to every regular curve composing $\partial \partial B$, and the vector $t_{\partial \partial B}$ denoting the tangent vector of the generic edge concurring in the considered wedge we get 


$$
\begin{gathered}
\int_{\partial \partial В} T(\partial \partial B, L) \cdot \nabla^{L} V= \\
=\sum_{J=1}^{L}(-1)^{L-J} \int_{\partial \partial B}\left(\left(\operatorname{div}_{\partial \partial B} \mathbb{P}_{\lambda}\right)^{L-J} T(\partial \partial B, L)\right)_{\perp} \cdot\left(\nabla^{J} V\right)_{\perp}+ \\
+(-1)^{L} \int_{\partial \partial B}\left(\left(\operatorname{div}_{\partial \partial B} \mathbb{P}_{\lambda}\right)^{L} T(\partial \partial B, L)\right) V+ \\
+\sum_{H=0}^{L-1}(-1)^{L-1-H} \int_{\partial \partial \partial B} \mathbb{P}_{\lambda}\left(\left(\operatorname{div}_{\partial \partial B} \mathbb{P}_{\lambda}\right)^{L-1-H} T(\partial \partial B, L)\right) \cdot t_{\partial \partial B} \cdot \nabla^{H} V
\end{gathered}
$$

Summing over $L$ and using once more associativity we get

$$
\begin{gathered}
\sum_{L=0}^{N-2} \int_{\partial \partial B} T(\partial \partial B, L) \cdot \nabla^{L} V= \\
=\sum_{J=1}^{N-2} \int_{\partial \partial B}\left(\sum_{L=J}^{N-2}(-1)^{L-J}\left(\operatorname{div}_{\partial \partial B} \mathbb{P}_{\lambda}\right)^{L-J} T(\partial \partial B, L)\right)_{\perp} \cdot\left(\nabla^{J} V\right)_{\perp}+ \\
+\int_{\partial \partial B}\left(\sum_{L=0}^{N-2}(-1)^{L}\left(\operatorname{div}_{\partial \partial B} \mathbb{P}_{\lambda}\right)^{L} T(\partial \partial B, L)\right) V+ \\
+\sum_{H=0}^{N-3} \int_{\partial \partial \partial B}\left(\sum_{L=H+1}^{N-2}(-1)^{L-1-H} \mathbb{P}_{\lambda}\left(\left(\operatorname{div}_{\partial \partial \partial} \mathbb{P}_{\lambda}\right)^{L-1-H} T(\partial \partial B, L)\right) \cdot t_{\partial \partial B}\right) \\
\cdot \nabla^{H} V
\end{gathered}
$$

\subsection{The terms of line (1-)forces and line $J+1$ - forces.}

Concerning the addend in 87 we easily recognize that it must be interpreted as a line density of 1 -force.

Therefore we introduce, in terms of introduced line stresses, the following

\section{Definition 9.11. Line density of 1-force}

$$
F(\partial \partial B, 0):=\sum_{L=0}^{N-2}(-1)^{L}\left(\operatorname{div}_{\partial \partial B} \mathbb{P}_{\lambda}\right)^{L} T(\partial \partial B, L)
$$

Regarding the addend in 86 , it naturally leads to the following 
Definition 9.12. Line density of $\mathrm{J}+1$-force

$$
F(\partial \partial B, J):=\left(\sum_{L=J}^{N-2}(-1)^{L-J}\left(\operatorname{div}_{\partial \partial B} \mathbb{P}_{\lambda}\right)^{L-J} T(\partial \partial B, L)\right)_{\perp}
$$

9.3 The terms of (1-)forces and $J+1$ - forces concentrated on wedges

The process of integration by parts is finally ended. We are left with forces, double forces, triple forces up to $N-3$ forces concentrated on wedges. Indeed the terms in 88 , naturally lead to the following

Definition 9.13. (1-) forces and $\mathbf{H}+1$-forces concentrated on wedges

$$
F(\partial \partial \partial B, 0):=\left(\sum_{L=1}^{N-2}(-1)^{L-1} \mathbb{P}_{\lambda}\left(\left(\operatorname{div}_{\partial \partial B} \mathbb{P}_{\lambda}\right)^{L-1} T(\partial \partial B, L)\right) . t_{\partial \partial B}\right)
$$

$$
\begin{gathered}
F(\partial \partial \partial B, H):=\left(\sum_{L=H+1}^{N-2}(-1)^{L-1-H}\right. \\
\mathbb{P}_{\lambda}\left(\left(\operatorname{div}_{\partial \partial B} \mathbb{P}_{\lambda}\right)^{L-1-H} T(\partial \partial B, L)\right) \cdot t_{\partial \partial B}
\end{gathered}
$$

In conclusion in the present subsection we have finally obtained the following

Representation formula for power expended by line stresses

$$
\begin{gathered}
\sum_{L=0}^{N-2} \int_{\partial \partial B} T(\partial \partial B, L) \cdot \nabla^{L} V=\sum_{J=1}^{N-2} \int_{\partial \partial B} F(\partial \partial B, J) \cdot\left(\nabla^{J} V\right)_{\perp}+ \\
+\int_{\partial \partial B} F(\partial \partial B, 0) V+\sum_{J=0}^{N-3} \int_{\partial \partial \partial B} F(\partial \partial \partial B, J) \cdot \nabla^{J} V
\end{gathered}
$$




\subsection{The obtained expression for Internal Power in $N-t h$ Gradi- ent Continua}

Gathering all results obtained in the previous subsection we get the searched representation formula for internal power

$$
\begin{gathered}
\mathcal{P}^{i n t}(B, V)=\int_{B} F(B, 0) V+\int_{\partial B} F(\partial B, 0) \cdot V \\
+\sum_{L=1}^{N-1} \int_{\partial B} F(\partial B, L) \cdot\left(\nabla^{L} V\right)_{\perp}+ \\
+\int_{\partial \partial B} F(\partial \partial B, 0) V+\sum_{J=1}^{N-2} \int_{\partial \partial B} F(\partial \partial B, J) \cdot\left(\nabla^{J} V\right)_{\perp}+ \\
+\sum_{J=0}^{N-3} \int_{\partial \partial \partial B} F(\partial \partial \partial B, J) \cdot \nabla^{J} V
\end{gathered}
$$

where all types of forces are represented in terms of bulk, surface and line stresses by means of the relationships which we recall for convenience in the following summary.

Remark 9.14. Surface stresses are defined in terms of bulk stresses and line stresses in terms of surface stresses by means of equations 78 and 66 . Therefore All kind of stresses are defined in terms of bulk stresses. This implies that all type of forces are represented in terms of bulk stresses.

We list now all introduced definitions for forces and stresses

$$
\begin{gathered}
T(\partial B, L):=\left(\sum_{\Lambda=L+1}^{N}(-1)^{\Lambda-1-L} \operatorname{div}^{\Lambda-1-L} T_{\Lambda} \cdot n\right) \\
T(\partial \partial B, J):=\cdot\left(\sum_{L=J+1}^{N-1}(-1)^{L-1-J} \mathbb{P}_{\sigma}\left(\left(d i v_{\partial B} \mathbb{P}_{\sigma}\right)^{L-1-J} T(\partial B, L)\right) \cdot \nu\right) \\
F(\partial B, 0):=\sum_{L=0}^{N-1}(-1)^{L}\left(\operatorname{div}_{\partial B} \mathbb{P}_{\sigma}\right)^{L} T(\partial B, L) \\
F(\partial B, J):=\left(\sum_{L=J}^{N-1}(-1)^{L-J}\left(\operatorname{div}_{\partial B} \mathbb{P}_{\sigma}\right)^{L-J} T(\partial B, L)\right)_{\perp}
\end{gathered}
$$




$$
\begin{array}{r}
F(\partial \partial B, 0):=\sum_{L=0}^{N-2}(-1)^{L}\left(\operatorname{div}_{\partial \partial B} \mathbb{P}_{\lambda}\right)^{L} T(\partial \partial B, L) \\
F(\partial \partial B, J):=\left(\sum_{L=J}^{N-2}(-1)^{L-J}\left(\operatorname{div}_{\partial \partial B} \mathbb{P}_{\lambda}\right)^{L-J} T(\partial \partial B, L)\right)_{\perp} \\
F(\partial \partial \partial B, H):=\left(\sum _ { L = H + 1 } ^ { N - 2 } ( - 1 ) ^ { L - 1 - H } \mathbb { P } _ { \lambda } \left(\left(\operatorname{div}_{\partial \partial B} \mathbb{P}_{\lambda}\right)^{L-1-H}\right.\right. \\
T(\partial \partial B, L)) \cdot t_{\partial \partial B}
\end{array}
$$

Finally it is easy to prove the following corollaries:

Corollary 9.15. The tensor $T_{N}$ determines the surface $N-1$ forces, the edge $N-2$ forces and the wedge $N-3$ forces as established by means of the following formulas:

$$
\begin{gathered}
F(\partial B, N-1)=T(\partial B, N-1)=\left(T_{N} \cdot n\right)_{\perp} \\
F(\partial \partial B, N-2):=T(\partial \partial B, n-2)=\left(\mathbb{P}_{\sigma}\left(T_{N} \cdot n\right) \cdot \nu\right)_{\perp} \\
\left.\left.F(\partial \partial \partial B, N-3)=\left(\mathbb{P}_{\lambda}\left(\mathbb{P}_{\sigma}\left(T_{N} \cdot n\right)\right) \cdot \nu\right)\right) \cdot t_{\partial \partial B}\right)
\end{gathered}
$$

Clearly

Corollary 9.16. The set of equations 96, 97, 98, 99 and 100 allow us to define three functionals mapping the contact surface $\partial B$ and the $N$ - tuple of tensor fields $\left\{T_{\Lambda}\right\}$ on to the set of corresponding contact actions

$$
\left\{\begin{array}{c}
(F(\partial B, J), J=0, \ldots N-1) \\
F(\partial \partial B, J), J=0, \ldots N-1 \\
F(\partial \partial \partial B, J), J=0, \ldots, N-2
\end{array}\right\}
$$

This map, for a fixed contact surface $\partial B$, is linear. We will denote the introduced linear functionals with the symbols introduced by means of the following definitions $\mathfrak{F}(\partial B), \mathfrak{F}(\partial \partial B)$ and $\mathfrak{F}(\partial \partial \partial B)$

$$
\begin{aligned}
\mathfrak{F}\left(\partial B,\left\{T_{\Lambda}\right\}, J\right) & :=F(\partial B, J) \\
\mathfrak{F}\left(\partial \partial B,\left\{T_{\Lambda}\right\}, J\right) & :=F(\partial \partial B, J) \\
\mathfrak{F}\left(\partial \partial \partial B,\left\{T_{\Lambda}\right\}, J\right) & :=F(\partial \partial \partial B, J)
\end{aligned}
$$

Proof. Simply consider the previous formulas 100, 96 and 97 and evaluate them when $H=N-3, L=N-2, L=N-1$. 
We will use the previous corollaries later, when we will consider the consequence of the Cauchy tetrahedron argument on the structure of contact actions in $N-t h$ gradient continua.

\subsection{Some final considerations about obtained results:}

Using the Postulate 8 and assuming that the bulk, surface and line stresses are sufficiently regular so that all needed divergences can be estimated, then from the representation formulas 94 and 57 and arbitrariness of test functions $V$ we can get the following "strong" version of the Principle of Virtual Powers

$$
\begin{gathered}
F(B, 0)=E \\
\forall L=0, \ldots N-1 \quad F(\partial B, L)=F_{L} \\
\forall J=0, \ldots N-2 \quad F(\partial \partial B, J)=G_{J} \\
\forall J=0, \ldots N-3 \quad F(\partial \partial \partial B, J)=H_{J}
\end{gathered}
$$

The first of the listed equations is a system of PDE defined inside the reference configuration of the body $B$, while the others are appropriate boundary conditions to be verified on regular surfaces, edges and wedges of the topological boundary of $B$.

We explicitly remark that

- From equations 98 and 96 it is evident the very particular nature of so called "Cauchy Postulate". Indeed the contact force per unit surface at any regular part of a surface regarded as a Cauchy cut in general do not depend only on the normal $n$ of such surface. In $N-t h$ gradient continua such contact force in general depends also on $\nabla n$ up to $\nabla^{N-1} n$.

- From equations 99,96 and 97 it is evident that line contact force depends in general on the vectors and tensors $\nu$ and $\nabla^{L} n$ relative to all regular surfaces concurring on considered edge, and on their derivatives along the edges.

- Concentrated $J$-forces are present at wedges depending on the geometry of concurring edges and regular surfaces, and more precisely on edges tangent vectors, on surface normals and their gradients, on edges exterior normals and their gradients.

In the present section we have completely characterized the structure of contact actions in $N-t h$ gradient continua by using the first method delineated in the section 2 . 


\section{Bodies, Contact Surfaces and Shapes ??}

In this section we start attacking the problem of characterizing the structure of contact actions in $N-t h$ gradient continua by using the second method delineated in the section 2: in other words we use the approach à la Cauchy.

Following Truesdell (1977) we call domains the closures of open Kellogg (1929) regular regions. We want to describe the contact actions exerted on a body, identified with the domain $\mathcal{C}$ it occupies in Euclidean space, through its topological boundary $\mathcal{S}:=\partial \mathcal{C}$ (which we will call the contact surface).

Only when we will need to represent with integral formulas the power expended by contact actions we will choose to limit our attention to a particular class of contact surfaces: those which are piecewise regular surfaces with edges and wedges, as defined in the previous section 4.

Remark 10.1. The definition of piecewise regular surface with edges and wedges will need to be generalized. In a forthcoming paper the present analysis will be generalized and we will introduce edges and wedges of order $k+1$, i.e. curves (edges) on piecewise regular surfaces on which the $k-t h$ order gradient of the normal suffers a jump (discontinuity of the first kind, or jump discontinuity), and points (wedges) at which edges of order $k$ are concurring.

Remark 10.2. The reasonings developed in the previous section 4.prove that on edges and wedges of order $k+1$ there may be concentrated $h$-forces with $h \geq k+1$.

We are interested in the dependence of contact actions on the shape of $\mathcal{S}$.

Then we have to define precisely what we call shape of $\mathcal{S}$ : it will be a local concept.

Definition 10.3. Surfaces locally having the same shape We say that the shape of the contact surface $\mathcal{S}$ at the point $x \in \mathcal{S}$ is the same as the shape of the contact surface $\mathcal{S}^{\prime}$ at the point $x^{\prime} \in \mathcal{S}^{\prime}$ if and only if there exist two open sets $I(x)$ and $I\left(x^{\prime}\right)$ such that

$$
\text { i) } \left.\quad x \in I(x), x^{\prime} \in I\left(x^{\prime}\right) \quad \text { and } i i\right) \quad t_{x^{\prime}-x}(I(x) \cap \mathcal{S})=I\left(x^{\prime}\right) \cap \mathcal{S}^{\prime}
$$

where $t_{u}$ denotes the translation of points, vectors and sets induced by the vector $u$. To say that the shape of the contact surface $\mathcal{S}$ at the point $x \in \mathcal{S}$ is the same as the shape of the contact surface $\mathcal{S}^{\prime}$ at the point $x^{\prime} \in \mathcal{S}^{\prime}$ we will occasionally use the notation

$$
(x, \mathcal{S}) \mathfrak{R}\left(x^{\prime}, \mathcal{S}^{\prime}\right)
$$


Definition 10.4. Shapes We call shape of $\mathcal{S}$ at the point $x \in \mathcal{S}$ the equivalence class with respect to the above defined equivalence relation $\mathfrak{R}$ to which belongs the ordered pair $(x, \mathcal{S})$.We denote this equivalence class by means of the symbol $[(x, \mathcal{S})]$.

Remark 10.5. Note that, according to this definition, when a surface is rotated it do change its shape.

Definition 10.6. Sets of shapes: Let $\mathcal{S}$ be a contact admissible surface. We introduce the set of the shapes of $\mathcal{S}$

$$
\Phi(\mathcal{S}):=\{[(x, \mathcal{S})] / \quad x \in \mathcal{S}\}
$$

We will also need to consider the set $\Phi$ of all admissible shapes:

$$
f \in \Phi \Leftrightarrow(\exists \mathcal{S} \text { admissible })(f \in \Phi(\mathcal{S}))
$$

Definition 10.7. Plane shapes Obviously the shape of a plane $\pi$ at any of its points depends only on the normal $n$ to the plane. When there is no ambiguity, we denote the shape of a plane simply by $n$.

Definition 10.8. Dihedral shapes Let us consider a non-degenerate dihedron. We denote by $n_{1}$ and $n_{2}$ the external normals to the half-planes forming it, and by $\tau$ the unit vector tangent to the edge of the dihedron, i.e. the line which is the intersection of the two half-planes. We assume that the ordered triple $\left(n_{1}, n_{2}, \tau\right)$ verifies the condition

$$
\left(n_{2} \times n_{1}\right) \cdot \tau>0 .
$$

Each of considered two half-planes is a two-dimensional manifold with border: the outer normal to this border is given respectively by the vectors $\nu_{1}=n_{1} \times \tau$ and $\nu_{2}=-n_{2} \times \tau$. On the edge of such a dihedron, the shape is constant and is determined by $n_{1}, n_{2}$ and $\tau$. This shape will be denoted by $\left(n_{1}, n_{2}, \tau\right)$. Note that $\left(n_{1}, n_{2}, \tau\right)=\left(n_{2}, n_{1},-\tau\right)$. The angle $\left(-n_{1}, n_{2}\right)$ in the plane oriented by $\tau$ will be called the dihedral angle of $\left(n_{1}, n_{2}, \tau\right)$; it is different from $0, \pi$ and $2 \pi$.

Definition 10.9. Nondegenerate $k$-tuple of vectors. Let us consider a point $x$ and a $k$-tuple of vectors $\left(n_{1}, \ldots, n_{k}\right)$ applied at $x$. The planes $\pi_{i}$ are defined by the conditions: i) $x \in \pi_{i}$ and ii) $n_{i} \perp \pi_{i}$. The unit tangent vector $\tau_{i}$ to the the edge $\gamma_{i}:=\pi_{i} \cap \pi_{i+1}$ is given by $\tau_{i}=n_{i} \times n_{i+1}$ (we define $\left.n_{k+1}:=n_{1}\right)$.Let us denote $P_{\pi}$ the projection operator in the plane $\pi$. The $k$-tuple $\left(n_{1}, \ldots, n_{k}\right)$ of vectors is said to be nondegenerate when there 
exists a plane $\pi$ such that i) $x \in \pi$, ii) for a unit vector $e$ in $\pi$ the following property holds

$$
(\forall i \leq k)(\forall j \leq k)\left((i<j) \Rightarrow\left(0<\frac{P_{\pi} \tau_{i}}{\left\|P_{\pi} \tau_{i}\right\|} \cdot e<\frac{P_{\pi} \tau_{j}}{\left\|P_{\pi} \tau_{j}\right\|} \cdot e\right)\right) .
$$

Definition 10.10. (Nondegenerate) Polihedral Wedge Shapes or Multiple planar shapes with edges concurring in a wedge. Let us consider a point $x$ and a nondegenerate $k$-tuple of vectors $\left(n_{1}, \ldots, n_{k}\right)$ applied at $x$.We call $A_{i}$ the angle having vertex in $x$ and as sides the half-lines originating from $x$ in the direction of $\tau_{i}$. Let us consider the piecewise regular surface $\bigcup_{i} A_{i}$. We call polihedral wedge shape or multiple planar shape with edges concurring in a wedge the equivalence class $\left[\left(x, \bigcup_{i} A_{i}\right)\right]$. When this will not lead to confusion we will denote a polihedral wedge shape with the symbol $\left(\widehat{n_{1}, \ldots, n_{k}}\right)$.

Definition 10.11. Cuts. Let $D_{1}, \ldots D_{p}$ be $p$ compact domains having as (topological) boundaries the contact surfaces denoted $\mathcal{S}_{1}, \ldots, \mathcal{S}_{p}$. Let $\mathcal{S}^{\cap}$ be the topological boundary of $\bigcap_{i=1}^{p} D_{i}$. At each point $x$ in $\bigcap_{i=1}^{p} \mathcal{S}_{i} \cap \mathcal{S}^{\cap}$ the shape of $\mathcal{S}^{\cap}$ depends only on the shape of all $\mathcal{S}_{i}$. For every $x \in \bigcap_{i=1}^{p} \mathcal{S}_{i} \cap \mathcal{S}^{\cap}$ we denote the shape of $\bigcap_{i=1}^{p} \mathcal{S}_{i} \cap \mathcal{S}^{\cap}$ at $x$ by the symbols

$$
\operatorname{Cut}\left(\left[\left(x, \mathcal{S}_{i}\right)\right], i=1, . ., p\right) ; \quad \text { or } \operatorname{Cut}\left(\left[\left(x, \mathcal{S}_{1}\right)\right],\left[\left(x, \mathcal{S}_{2}\right)\right], \ldots,\left[\left(x, \mathcal{S}_{p}\right)\right]\right) \text {. }
$$

Example 10.12. Plane Cuts. Let $D$ be a domain the boundary of which is the contact surface $\mathcal{S}$. Let $\pi$ be a plane and $H$ the half-space with boundary $\pi$ and outer unit normal $n$. Let $x \in \pi \cap \mathcal{S}$. We will call the $\operatorname{Cut}([(x, \mathcal{S})],[(x, \pi)])$ a plane cut and we will shortly denote it by $\operatorname{Cut}([(x, \mathcal{S})], n)$.

Example 10.13. Multiple Plane Cuts. Let $D$ be a domain the boundary of which is the contact surface $\mathcal{S}$. Let $\pi_{i} ; i=1, \ldots p$ be planes having as outer unit normal the vectors $n_{i}$ respectively. Let $x \in \bigcap_{i=1}^{p} \pi_{i} \cap \mathcal{S}$. We will call the $C u t\left([(x, \mathcal{S})],\left[\left(x, \pi_{1}\right)\right], . .,\left[\left(x, \pi_{p}\right)\right]\right)$ a plane cut and we will shortly denote it by $C u t\left([(x, \mathcal{S})], n_{1}, n_{2}, \ldots, n_{p}\right)$.

Definition 10.14. Admissible domains and contact surfaces. We only consider domains the topological boundary of which $\mathcal{S}$ (contact admissible surface) is piecewise regular. In other words we assume that i) $\mathcal{S}$ 
is a finite union of two-dimensional suitably regular compact manifolds with border (called the faces of $\mathcal{S}$ ) ii) the union of the borders of these faces is a finite union of one-dimensional suitably regular compact manifolds with boundary (called the edges of $\mathcal{S}$ ), iii) the edges are concurring in wedges and iv) the set of wedges is finite.

Definition 10.15. Regular points of contact surfaces All internal points of the faces (i.e. those points which do not belong to the border of the faces) are called regular points of the face, or points where $\mathcal{S}$ has a regular shape. The set of regular points is denoted by $\mathcal{S}_{r}$; the set of all internal points of the edges (i.e. those points which do not belong to the border of the faces) is denoted by $\mathcal{L}_{r}$. An internal point of an edge is also called regular point of the edge, or point where $\mathcal{S}$ has an edge shape. The set of all wedges will be denoted by $\mathcal{W}_{S}$. Moreover, we assume that, i) everywhere in $\mathcal{L}_{r}, \mathcal{S}$ is tangent to a non degenerate dihedron and ii) at every wedge in $\mathcal{S}$ there exists a tangent polihedral plane surface (i.e. a surface having a polihedral wedge shape or, which is the same, a multiple planar shape with edges concurring in it).

Remark 10.16. When we say that at a edge regular point the contact surface is tangent to a nondegenerate dihedron we mean that the two regular surfaces concurrent at the edge have both a tangent plane and that these two plane form a nondegenerate dihedron. Similarly when we say that at every wedge in $\mathcal{S}$ there exists a tangent polihedral plane surface we mean that every edge concurring in the wedge has a tangent line and that every regular surface concurring in the wedge has a tangent plane and the set of these tangent lines and planes form a surface having a polihedral wedge shape.

Definition 10.17. We denote by $\Phi^{F}(\mathcal{S})$ the set of all face shapes of $\mathcal{S}$, by $\Phi^{E}(\mathcal{S})$ the set of all edge shapes of $\mathcal{S}$ and by $\Phi^{W}(\mathcal{S})$ the set of all wedge shapes of $\mathcal{S}$ : in formulas

$$
\begin{gathered}
\Phi^{F}(\mathcal{S}):=\left\{[(x, \mathcal{S})], x \in \mathcal{S}_{r}\right\} ; \quad \Phi^{E}(\mathcal{S}):=\left\{[(x, \mathcal{S})], x \in \mathcal{L}_{r}\right\} \\
\Phi^{W}(\mathcal{S}):=\left\{[(x, \mathcal{S})], x \in \mathcal{W}_{S}\right\} .
\end{gathered}
$$

We remark that in this way we have found a partition of the set of shapes of a contact admissible surface:

$$
\Phi(\mathcal{S})=\Phi^{F}(\mathcal{S}) \cup \Phi^{E}(\mathcal{S}) \cup \Phi^{W}(\mathcal{S})
$$

Definition 10.18. Prescribed shapes: A set of shapes $E$ is called a set of prescribed shapes if there exists a finite set $\left\{\mathcal{S}_{1}, \mathcal{S}_{2}, \ldots \mathcal{S}_{m}\right\}$ of compact admissible contact surfaces such that $E \subseteq \bigcup_{i=1}^{m} \Phi\left(\mathcal{S}_{i}\right)$. 
The following examples should make clearer the meaning of the just introduced definition.

Example 10.19. The set of images $C_{t}$ of a cube $C_{1}$ under a family of homothetic transformations of ratio $t \in] 0,1]$ is a set of prescribed shapes;

Example 10.20. The set of images $C_{t}^{\prime}$ of a cube $C_{1}$ under a family of rotations of angle $t \in[0, \pi]$ around a given axis is not a set of prescribed shapes;

Example 10.21. The family of spheres $S_{t}$ of centre $x$ and radius $\left.\left.t \in\right] 0,1\right]$ has not prescribed shapes.

Definition 10.22. Let us consider a finite set $\left\{\mathcal{S}_{1}, \mathcal{S}_{2}, \ldots \mathcal{S}_{m}\right\}$ of compact admissible surfaces and a finite set of unit vectors $U:=\left\{u_{1}, \ldots, u_{M}\right\}$. A set of shapes $E$ is called a set of prescribed (multiple) plane cuts

$$
E \subseteq \bigcup_{l=1}^{M}\left\{C u t\left(f, u_{1}, \ldots, u_{l}\right) ; f \in \Phi\left(\mathcal{S}_{i}\right) ; u_{i} \in U, i=1, \ldots, l\right\}
$$

In what follows we will need to consider functions defined in some set of shapes. It would be desirable to introduce in the set of shapes a topological structure: in the present context we refrain from such a task which is really challenging. Instead, by introducing the concept of "set of prescribed shapes" we have considered a kind of "compactness" in the set of all admissible shapes. This definition will reveal itself to be sufficient to our aims.

\subsection{Contact Actions}

In this paper we consider the class of continua which were introduced by Green and Rivlin (1964) or Mindlin (1965). In these continua there are contact actions which are not forces. An example of such generalized continua is given by those studied by Germain, which called them second gradient continua, as their deformation energy density depends not only on the first gradient of displacement but also on the second gradient of displacement. However in generalized continua forces represent an important class of contact actions the properties of which are somehow different from those of the other contact actions.

As first step we will start by accepting the following:

\section{Generalized Cauchy Postulate: Part I}


Let us consider a continuous body $B$ occupying, in a given configuration, a domain $D$ having as topological boundary the admissible contact surface $S$. Let $D$ the set of admissible virtual displacement of $B$ starting from the considered configuration. Each of these displacements is a field defined in $D$ and having trace on $S$ and is a vector valued test function. We assume that the power expended by contact actions exerted on $B$ is a distribution in the sense of Schwartz.

This postulate has been already introduced in continuum mechanics (in a slightly less general form) at least already by Cosserat brothers (1908)(1909). A detailed historic review of the relevant literature can be found e.g. in Maugin (1980). In the textbook of Salençon (1988-2005) this postulate is presented in a attractive didactical form.

Because of the just introduced postulate we can be guided in the formulation of the needed definitions by the already cited (see previous sections) representation theorem for distributions due to Laurent Schwartz (quoted in the previous section 4).

Therefore we start by considering contact actions which indeed are (1-) forces and then proceed by considering $k$-forces.

Definition 10.23. Surface and Line density of contact (1-)forces. Contact (1-)forces concentrated on wedges. Let us consider any admissible domain $D$.Its boundary $\mathcal{S}$ is a contact piecewise regular surface, including edges and wedges. We call contact (1-)force a contact action for which the power expended on a virtual velocity field $V$ is given by the following expression

$$
\mathcal{P}_{0}^{c o n}(V)=\int_{\mathcal{S}_{r}} \mathrm{~F}_{0}^{s} V+\int_{\mathcal{L}_{r}} \mathrm{~F}_{0}^{l} V+\int_{\mathcal{W}_{S}} \mathrm{~F}_{0}^{c} V .
$$

the vector functions, $\mathrm{F}_{0}^{s}, \mathrm{~F}_{0}^{l}$ and $\mathrm{F}_{0}^{c}$ defined respectively on the faces, the edges and the wedges of $\mathcal{S}$ are called i) surface density of contact (1-)forces, ii) line density of contact (1-)forces, iii) contact (1-)forces concentrated on wedges.

Remark 10.24. In the following we will be willing to consider surface contact forces depending continuously on curvature. We will assume that such forces densities are bounded on a set of prescribed shapes but not on the set of all admissible shapes.

Definition 10.25. Surface and Line density of contact $k$-forces. Contact $k$-forces concentrated on wedges. Let us consider any admissible domain $D$.Its boundary $\mathcal{S}$ is a contact piecewise regular surface, 
including edges and wedges. We call contact $(k-)$ force a contact action for which the power expended on a virtual velocity field $V$ is given by the following expression (where we used the notations introduced in the previous section 4)

$$
\mathcal{P}_{k}^{c o n}(V)=\int_{\mathcal{S}_{r}} \mathrm{~F}_{k}^{s} \cdot\left(\nabla^{k} V\right)_{\perp}+\int_{\mathcal{L}_{r}} \mathrm{~F}_{k}^{l} \cdot\left(\nabla^{k} V\right)_{\perp}+\int_{\mathcal{W}_{S}} \mathrm{~F}_{k}^{c} \cdot \nabla^{k} V .
$$

the tensor functions, $\mathrm{F}_{k}^{s}, \mathrm{~F}_{k}^{l}$ and $\mathrm{F}_{k}^{c}$ defined respectively on the faces, the edges and the wedges of $\mathcal{S}$ are called i) surface density of contact $k$-forces, ii) line density of contact $k$-forces, iii) contact $k$-forces concentrated on wedges.

On occasion we will denote a generic vector or tensor field in the set $\left\{\mathrm{F}_{k}^{s}, \mathrm{~F}_{k}^{l}, \mathrm{~F}_{k}^{c} ; k=0, \ldots, L\right\}$ simply with the symbol $\mathrm{F}$,adding some super or sub script when needed.

All the considerations which we will develop in this work are based on the following further hypothesis:

\section{Generalized Cauchy Postulate: Part II}

Let us consider a body, occupying a region $\mathcal{C}$ included in the Euclidean three-dimensional space and denote by $\mathcal{V}$ its space of translations. We assume that all tensor functions $\mathrm{F}$ previously introduced are univocally determined in terms of the position $x$ and on the shape of $\mathcal{S}$ at $x$.

In other words:

we assume that there exists a function $\hat{\mathrm{F}}_{k}$ (which we will call $k-t h$ order stress function) of the two independent variables $x$, and $f$, where $f$ is a shape

$$
\hat{\mathrm{F}}_{k}: \quad(x, f) \in \mathcal{C} \times \oplus \mapsto \hat{\mathrm{F}}_{k}(x, f) \in \underbrace{\mathcal{V} \otimes \ldots \otimes \mathcal{V}}_{k \text { times }}
$$

such that

$$
\mathrm{F}_{k}(x)=\hat{\mathrm{F}}_{k}(x,[(x, \mathcal{S})]) .
$$

Definition 10.26. We call generalized stress state of the considered body the set of the functions $\hat{F}$ the existence of which we have just postulated.

Remark 10.27. We do not assume uniform boundedness of all tensor partial functions $\hat{\mathrm{F}}(x, \cdot)$ in the set of all shapes $\Phi$, i.e. -once $x$ is fixed- with respect to the variable $f$. 
Remark 10.28. Because of the previous definition the fields $\mathrm{F}_{k}^{l}$ may depend not only on the geometry of the edge itself, but also on the "limit properties" of the faces common border of which is the considered edge.

Remark 10.29. Similarly the $k$-forces $\mathrm{F}_{k}^{c}$ depend on all the "limit properties" of the faces and edges concurring in the considered wedge.

In the same way as the Cauchy's construction of the stress tensor presumes the continuity of contact forces, our construction will require a list of

\section{Regularity assumptions.}

The functions $\hat{F}$ verifies all the conditions which follows:

1. Let $\mathcal{S}_{F}$ be a face and $\mathcal{L}$ an edge of an admissible contact surface $\mathcal{S}$. The functions

$$
x \in \mathcal{S}_{F} \mapsto \hat{\mathrm{F}}^{s}\left(x,\left[\left(x, \mathcal{S}_{F}\right)\right]\right) ; \quad x \in \mathcal{L} \mapsto \hat{\mathrm{F}}^{l}(x,[(x, \mathcal{L})])
$$

are continuous.

2. Let $E$ be a set of prescribed shapes or prescribed plane cuts. We partition $E$ into three disjoint subsets, $E^{l}, E^{s}, E^{c}$ which contain respectively the edge shapes, the regular shapes and the wedge shapes in $E$. For a suitably fixed shape $f$ (i.e. for a regular, edge or wedge shape depending on the type of $\hat{F}$ we are considering) we can introduce the partial function

$$
\hat{\mathrm{F}}(\cdot, f): x \mapsto \hat{\mathrm{F}}(x, f) .
$$

We assume the equi-continuity of the families of functions

$$
\left\{\hat{\mathrm{F}}^{s}(\cdot, f), f \in E^{s}\right\}, \quad\left\{\hat{\mathrm{F}}^{l}(\cdot, f), f \in E^{l}\right\}, \quad\left\{\hat{\mathrm{F}}^{c}(\cdot, f), f \in E^{c}\right\}
$$

that is, we assume that (the superscript $\alpha$ can assume the values $l, s$, c)

$$
\begin{gathered}
(\forall \varepsilon>0)(\exists \eta>0)\left(\forall x_{0}\right)(\forall x)\left(\forall f \in E^{\alpha}\right) \\
\left(\left\|x-x_{0}\right\|<\eta \Rightarrow\left\|\hat{\mathrm{F}}^{\alpha}\left(x_{0}, f\right)-\hat{\mathrm{F}}^{\alpha}(x, f)\right\|<\varepsilon\right) .
\end{gathered}
$$

3. Let $\mathcal{S}_{F}$ be a given face of an admissible surface $\mathcal{S}$, let $u$ be a unit vector nowhere normal to $\mathcal{S}_{F}$. We assume that the function which maps the variables $x$ into the vector

$$
\hat{\mathrm{F}}^{l}\left(x, C u t\left(\left[\left(x, \mathcal{S}_{F}\right)\right], u\right)\right.
$$


is a continuous function. In formulas

$$
\left.\begin{array}{c}
(\forall \varepsilon>0)\left(\forall x_{0} \in \mathcal{S}_{F}\right)(\exists \delta>0)\left(\forall x \in \mathcal{S}_{F}\right) \\
\left\|x-x_{0}\right\|<\delta \Rightarrow \\
\Rightarrow \| \hat{\mathrm{F}}^{l}\left(x, \operatorname{Cut}\left(\left[\left(x, \mathcal{S}_{F}\right)\right], u\right)-\hat{\mathrm{F}}^{l}\left(x_{0}, C u t\left(\left[\left(x_{0}, \mathcal{S}_{F}\right)\right], u\right) \|<\varepsilon\right.\right.
\end{array}\right)
$$

4. Let $\mathcal{S}_{F}$ be a given face of an admissible surface $\mathcal{S}$, let $u_{1}, \ldots, u_{p}$ be unit vectors nowhere normal to $\mathcal{S}_{F}$. Let $\mathcal{L}$ be a given edge of an admissible surface $\mathcal{S}$, let $u_{1}, \ldots, u_{q}$ be unit vectors nowhere normal to $\mathcal{L}$. Let us assume that for $x \in \mathcal{S}_{F}$ and $y \in \mathcal{L}, C u t\left(\left[\left(x, \mathcal{S}_{F}\right)\right], u_{1}, \ldots, u_{p}\right)$ and $C u t\left([(y, \mathcal{S})], u_{1}, \ldots, u_{q}\right)$ are wedge shapes. We assume that the functions

$$
\begin{gathered}
F_{\mathcal{S}_{F}}:\left(x, u_{1}, \ldots, u_{p}\right) \mapsto \hat{\mathrm{F}}^{c}\left(x, \operatorname{Cut}\left(\left[\left(x, \mathcal{S}_{F}\right)\right], u_{1}, \ldots, u_{p}\right)\right. \\
F_{\mathcal{L}}:\left(y, u_{1}, \ldots, u_{q}\right) \mapsto \hat{\mathrm{F}}^{c}\left(y, \operatorname{Cut}\left([(y, \mathcal{S})], u_{1}, \ldots, u_{q}\right)\right.
\end{gathered}
$$

are continuous functions respectively with respect to the variables $x$ or $y$.

Proposition 10.30. Let $\mathcal{S}_{F}$ and $\mathcal{L}$ be the intersection with open subsets of a face and an edge included in an admissible surface $\mathcal{S}$, let $u$ be a unit vector nowhere normal to $\mathcal{S}_{F}$ and let $B$ be a compact set. Let us consider the following functions, determined by the generalized stress state $\hat{\mathrm{F}}$ :

$$
\begin{gathered}
F^{s}:(x, y) \in B \times\left(B \cap \mathcal{S}_{F}\right) \mapsto \hat{\mathrm{F}}^{s}(x,[(y, \mathcal{S})]) \\
F^{l}:(x, y) \in B \times(B \cap \mathcal{L}) \mapsto \hat{\mathrm{F}}^{l}(x,[(y, \mathcal{S})]) \\
F_{C u t}^{l}:(x, y) \in B \times\left(B \cap \mathcal{S}_{F}\right) \mapsto \hat{\mathrm{F}}^{l}(x, C u t([(y, \mathcal{S})], u)) . \\
F_{C u t, \mathcal{S}}^{c}:(x, y) \in B \times\left(B \cap \mathcal{S}_{F}\right) \mapsto \hat{\mathrm{F}}^{c}\left(x, \operatorname{Cut}\left(\left[\left(x, \mathcal{S}_{F}\right)\right], u_{1}, \ldots, u_{p}\right)\right. \\
F_{C u t, \mathcal{L}}^{c}:(x, y) \in B \times(B \cap \mathcal{L}) \mapsto \hat{\mathrm{F}}^{c}\left(y, \operatorname{Cut}\left([(y, \mathcal{S})], u_{1}, \ldots, u_{q}\right)\right.
\end{gathered}
$$

The regularity assumptions which we have accepted imply that $F^{s}, F^{l}$ and $F_{C u t}^{l}$ are uniformly continuous.

Proof. To prove this proposition for the function 127 let us consider $(x, y) \in$ $B \times\left(B \cap \mathcal{S}_{F}\right)$ and $(\bar{x}, \bar{y}) \in B \times\left(B \cap \mathcal{S}_{F}\right)$, the vector $u=x-y$, the point $\bar{y}^{\prime}=\bar{y}+u$ and the translated contact surface $\mathcal{S}^{\prime}:=t_{u}(\mathcal{S})$. Obviously as $(y, \bar{y}) \in \mathcal{S}_{F} \times \mathcal{S}_{F}$ then $x=y+u \in \mathcal{S}^{\prime}, \bar{y}^{\prime}=\bar{y}+u \in \mathcal{S}^{\prime},[(\bar{y}, \mathcal{S})]=\left[\left(\bar{y}^{\prime}, \mathcal{S}^{\prime}\right)\right]$ and 
$[(y, \mathcal{S})]=\left[\left(x, \mathcal{S}^{\prime}\right)\right]$. It is easy to verify that the following chain of equalities and inequalities hold

$$
\begin{aligned}
& \left\|\hat{\mathrm{F}}^{s}(x,[(y, \mathcal{S})])-\hat{\mathrm{F}}^{s}(\bar{x},[(\bar{y}, \mathcal{S})])\right\|= \\
= & \left\|\hat{\mathrm{F}}^{s}\left(x,\left[\left(x, \mathcal{S}^{\prime}\right)\right]\right)-\hat{\mathrm{F}}^{s}\left(\bar{x},\left[\left(\bar{y}^{\prime}, \mathcal{S}^{\prime}\right)\right]\right)\right\| \leq \\
\leq & \left\|\hat{\mathrm{F}}^{s}\left(x,\left[\left(x, \mathcal{S}^{\prime}\right)\right]\right)-\hat{\mathrm{F}}^{s}\left(\bar{y}^{\prime},\left[\left(\bar{y}^{\prime}, \mathcal{S}^{\prime}\right)\right]\right)\right\|+ \\
+ & \left\|\hat{\mathrm{F}}^{s}\left(\bar{y}^{\prime},\left[\left(\bar{y}^{\prime}, \mathcal{S}^{\prime}\right)\right]\right)-\hat{\mathrm{F}}^{s}\left(\bar{x},\left[\left(\bar{y}^{\prime}, \mathcal{S}^{\prime}\right)\right]\right)\right\| .
\end{aligned}
$$

Finally one gets the required uniform continuity by finding separately the upper bounds for the addends 134 and 135. The first of these upper bounds is easily found by recalling the assumed continuity (numbered item 1 . in the regularity assumptions) which becomes uniform continuity as the set $B$ is assumed to be compact. The second upper bound is estimated by using the equi-uniform continuity (numbered item 2.) when recalling that

$$
\left\|\bar{y}^{\prime}-\bar{x}\right\|=\|\bar{y}+u-\bar{x}\| \leq\|y-x\|+\|\bar{y}-\bar{x}\| .
$$

With similar arguments we can prove the statement for the functions 128, 129, 130 and.131.

Proposition 10.31. . Let us consider a family of admissible contact surfaces $\left\{\mathcal{S}_{\lambda}, \lambda \in I\right\}$ where $I$ is a suitable set of indices and the set of shapes

$$
\Phi_{I}:=\left\{f \in \Phi /(\exists \lambda \in I)\left(f \in \Phi\left(\mathcal{S}_{\lambda}\right)\right)\right\} .
$$

Let us assume that $\Phi_{I}$ is a set of prescribed shapes or prescribed plane cuts. Then the regularity hypotheses 1., 2. and 3. imply the uniform boundedness of the families of functions (where $k$ is the needed tensoriality order)

$$
\{\hat{\mathrm{F}}(\cdot, f): x \in \mathcal{C} \mapsto \hat{\mathrm{F}}(x, f) \in \underbrace{\mathcal{V} \otimes \ldots \otimes \mathcal{V}}_{k \text { times }}, \quad f \in \Phi_{I}\} .
$$

Proof. Indeed, Proposition 10.30 implies that, for every admissible surface $\mathcal{S}_{\lambda}$, the functions $127,128,129,130$ and 131 (in the definitions replace the faces, edges and wedges of $\mathcal{S}$ with those of $\mathcal{S}_{\lambda}$ ) are bounded in their domains of definitions. The proposition is then proven simply recalling the definitions of prescribed shapes and prescribed plane cuts. 
Remark 10.32. Proposition 10.31 states a kind of relative compactness of the families 137 (see the Corollary to the Arzelà-Ascoli theorem in Abraham, Marsden and Ratiu (1988)).

Remark 10.33. Our hypotheses allow for any continuous dependence of contact $k$-forces densities $\mathrm{F}$ on the curvature tensor, edge curvature or torsion or on any other higher order shape operator of the contact surface, at any surface, edge or wedge point.

\section{A Seeming Impossibility For Edge Forces}

This section is very close to section 3. of the paper dell'Isola and Seppecher (1997). It is aimed to persuade the reader of the validity of an important statement:

Not all types of Contact Actions are physically admissible.

Indeed we will start by proving that

If a stress state is constituted only by contact surface and line forces then it is physically admissible only when contact line forces are always vanishing.

We must, obviously, specify what we mean with the expression: physically admissible.

We will say that a stress state is physically admissible when it verifies the regularity assumptions $\mathbf{1 0 . 1}$ and quasi-balance of contact powers.

Quasi-balance of powers expended by contact actions is a condition which has solid physical grounds: it implies stringent restrictions upon the dependence of contact actions on shape It is a generalization of the hypothesis of 'quasi-balance of contact forces' formulated by Noll and Virga (1990). This last states that

\section{Hypothesis of quasi-balance of contact forces}

There exists a positive scalar $K$ such that, for any admissible domain $V$, the following inequality holds

$$
\left\|F^{c}(V)\right\|<K|V| .
$$

In (? ) it is also assumed the following assumptions

Contact actions reduce to surface and edge forces 
In formulas:

$$
F^{c}(V)=\int_{\mathcal{S}^{r}} \hat{\mathrm{F}}_{0}^{s}(x,[(x, \mathcal{S})]) d s+\int_{\mathcal{L}^{r}} \hat{\mathrm{F}}_{0}^{l}(x,[(x, \mathcal{S})]) d l .
$$

Using the language which we have introduced in this work: in the paper Noll and Virga (1990) it is assumed that

i) the stress state is e completely specified by the two functions

$$
\hat{\mathrm{F}}_{0}^{s}, \quad \hat{\mathrm{F}}_{0}^{l}
$$

ii) the only physically allowed stress states are those for which condition 138 holds.

In this section we prove that by means of its theoretical framework actually the aforementioned paper does not manage to abandon the Cauchy format of continuum mechanics.

Instead: to give a framework where the searched generalization can be obtained we must withdraw from the format of mechanics in which the most fundamental concept is that of "force" (so called Newtonian Axiomatics) and we must embrace the point of view of D'Alembert whose Axiomatics is based on the fundamental concept of power and who considers the concept of force as a derived one.

By following the beautiful presentation of continuum mechanics due to Salençon (1988-2005) we improve the preceding hypothesis 138, assuming that

\section{Hypothesis of quasi-balance of power expended by contact actions}

The power $\mathcal{P}_{U}^{c}$ of contact actions distribution expended on any $C^{\infty}$ velocity field $U$ is quasi-balanced. This means that, for any $C^{\infty}$ field $U$, we assume the existence of a positive $K_{U}$ such that, for any admissible domain $V$, the following inequality holds

$$
\left|\mathcal{P}_{U}^{c}\right|<K_{U}|V|
$$

Remark 11.1. Everybody who wants to develop an axiomatization of mechanics must be willing to use power at least as a derived concept. Indeed nobody is able to refrain from the use of the concept of power or may doubt about the validity of equation 141 . The most faithful followers of so called Newtonian approach may be willing to deduce (!) 141 from a most fundamental (!) assumption but nobody can dare to state that it should not be accepted as valid. 
Remark 11.2. The condition expressed by (138) is implied by the one expressed by (142). This can be simply verified by considering that, when considering in it three linearly independent constant fields, formula (142) reduces to $(138)$.

Once we accept the hypothesis 140 about contact actions then the hypothesis 141 reads:

\section{Quasi balance of Powers for Contact Actions reducing to Surface and Line Forces}

$$
\left|\mathcal{P}_{U}^{c}(V)\right|=\left|\begin{array}{c}
\int_{\mathcal{S}^{r}} \hat{\mathrm{F}}_{0}^{s}(x,[(x, \mathcal{S})]) \cdot U(x) d s \\
+\int_{L^{r}} \hat{\mathrm{F}}_{0}^{l}(x,[(x, \mathcal{S})]) \cdot U(x) d l
\end{array}\right|<K_{U}|V|
$$

for every field $U,(v \cdot w$ denotes the inner product between the vectors $v$ and $w)$.

Remark 11.3. The dependence of $K_{U}$ on $U$ will be immaterial in what follows, as in our arguments the field $U$ is always kept fixed.

In this section we want to show that 142 implies that on every edge $\mathcal{L}^{r}$ of a contact surface $\mathcal{S}$

$$
\hat{\mathrm{F}}_{0}^{l}(x,[(x, \mathcal{S})])=0 .
$$

This means that actually by assuming 142 one does not obtain any generalization of Cauchy format of continuum mechanics.

In the language which we have introduced in this work one can state that: Quasi-balance of contact power and the assumption 140 about stress state implies that no contact edge forces are possible.

Remark 11.4. The quasi-balance of moment of forces can be obtained from condition 142 by considering three independent spins.

As hypothesis (142) is stronger than (138) it will imply more stringent restrictions upon the dependence of contact actions on shape.

Our goal now is to study its consequences on the functions $\hat{F}$. We begin by considering edges whose shape is dihedral; we will then extend our results to general edges. 


\subsection{Condition 142 implies that Forces on Dihedral Edges must vanish.}

From the point of view of the logical flow of the presentation, stricto sensu, the reader can simply jump this section. However the rigorous argument which is presented here gives a more intuitive ground to the following Theorem 11.7.

In this subsection we see how quasi balance of power expended by contact surface and line forces implies that line forces must vanish.

Theorem 11.5. For stess states of the form 140, and verifying the regularity assumption 10.1 inequality (142) is incompatible with nonzero contact line forces on dihedral edges: indeed (142) implies that for any dihedral shape $d$, the following equality holds

$$
\hat{\mathrm{F}}_{0}^{l}(\cdot, d)=0 .
$$

Proof. Let $d=\left(n_{1}, n_{2}, \tau\right)$.

We use the orthogonal coordinate system $\left(x_{0} ; e_{1}, e_{2}, e_{3}\right)$, with

$$
e_{2}=\tau, \quad e_{3}=\frac{n_{1}+n_{2}}{\left\|n_{1}+n_{2}\right\|}
$$

We consider a family of domains, parametrized by the set of integers greater than a positive $N$. The general element $V_{N}$ (the boundary of which we denote by $S_{N}$ ) of this family is a thin slab with a grooved surface (see Figure 1 in dell'Isola and Seppecher (1997)). This domain is a polyhedron conceived in such a way that the set of shapes of its boundary is finite and is independent of $N$ (This set contains exactly 7 different plane shapes and 16 different dihedral shapes.) Its volume $\left|V_{N}\right|$ is of the same order as $N^{-4}$ the total area of its boundary $\left|S_{N}\right|$ is of the same order as $N^{-2}$, when $N$ tends to infinity. Let us define the following unions of edges:

$$
\begin{aligned}
& L_{N}^{1}=\left\{x \in S_{N}:[(x, S)]=\left(n_{1}, n_{2}, \tau\right)\right\} \\
& L_{N}^{2}=\left\{x \in S_{N}:[(x, S)]=\left(n_{2}, n_{1}, \tau\right)\right\}
\end{aligned}
$$

and

$$
L_{N}^{3}=L_{N} \backslash\left(L_{N}^{1} \cup L_{N}^{2}\right) .
$$

The total length of $L_{N}^{3}$ is of the same order as $N^{-1}$ and the total lengths of $L_{N}^{1}$ and $L_{N}^{2}$ tend to 1 . Then the forces on the double array of edges $L_{N}^{1} \cup L_{N}^{2}$ are dominant. As the shapes of $V_{N}$ are prescribed, contact force 
densities are bounded independently of $N$. Inequality (138) applied to $V_{N}$ implies

$$
\lim _{N \longrightarrow \infty}\left\{\int_{L_{N}^{1}} \hat{\mathrm{F}}_{0}^{l}\left(x,\left(n_{1}, n_{2}, \tau\right)\right) d l+\int_{L_{N}^{2}} \hat{\mathrm{F}}_{0}^{l}\left(x,\left(n_{2}, n_{1}, \tau\right)\right) d l\right\}=0 .
$$

Using the mean value theorem for each component of the last equality and again the continuity of $\mathcal{F}$ with respect to $x$ we get an action-reaction principle

$$
\hat{\mathrm{F}}_{0}^{l}\left(x_{0},\left(n_{1}, n_{2}, \tau\right)\right)=-\hat{\mathrm{F}}_{0}^{l}\left(x_{0},\left(n_{2}, n_{1}, \tau\right)\right) .
$$

Consider the field

$$
U: x \mapsto\left(x . e_{3}\right) U_{0},
$$

$U_{0}$ being a fixed vector. On $V_{N}, N^{2} U$ is bounded independently of $N$. The same reasoning as before shows that inequality (142) implies

$$
\begin{aligned}
\lim _{N \longrightarrow \infty} & N^{2}\left\{\int_{L_{N}^{1}} \hat{\mathrm{F}}_{0}^{l}\left(x,\left(n_{1}, n_{2}, \tau\right)\right) \cdot U(x) d l\right. \\
& +\int_{L_{N}^{2}} \hat{\mathrm{F}}_{0}^{l}\left(x,\left(n_{2}, n_{1}, \tau\right)\right) \cdot U(x) d l=0 .
\end{aligned}
$$

On $L_{N}^{1}$ and $L_{N}^{2}, N^{2} U$ does not depend either on $N$ or on $x$ as it is equal respectively to $U_{0}$ and $2 U_{0}$. Then we obtain, because of arbitrariness of $U_{0}$,

$$
\lim _{N \longrightarrow \infty}\left\{\int_{L_{N}^{1}} \hat{\mathrm{F}}_{0}^{l}\left(x,\left(n_{1}, n_{2}, \tau\right)\right) d l+2 \int_{L_{N}^{2}} \hat{\mathrm{F}}_{0}^{l}\left(x,\left(n_{2}, n_{1}, \tau\right)\right) d l\right\}=0 .
$$

Using the continuity of $\hat{\mathrm{F}}_{0}^{l}$ with respect to $x$, the mean value theorem for each component of the previous equality and Equation (148) we get

$$
\hat{\mathrm{F}}_{0}^{l}\left(x_{0},\left(n_{1}, n_{2}, \tau\right)\right)=0
$$

Remark 11.6. This proof is not the simplest one can conceive (see the proof of the following Theorem 11.7). However, we present it here because it is suggestive: our construction shows that a limit of pairs of opposite edge forces cannot be quasi-balanced. This means that in generalized continua contact actions can include nonvanishing edge forces only if they also include nonvanishing surface 2-forces. More precisely: the theorem we have just proven shows how a sequence of systems of edge forces can converge to a surface double force: we have thus rigorously illustrated the terms 'double forces' and 'double normal traction' introduced by Germain (1973). 


\subsection{Condition 142 implies that Forces on General Edges must vanish.}

The Theorem proven in the previous subsection can be actually generalized to any edge of a contact surface.

Theorem 11.7. Let $\mathcal{S}$ be an admissible surface. For stess states of the form 140, and verifying the regularity assumptions 10.1, inequality (142) imply that, at every regular point $x_{0}$ of an edge, we have

$$
\hat{\mathrm{F}}_{0}^{l}\left(x_{0},\left[\left(x_{0}, \mathcal{S}\right)\right]\right)=0 .
$$

In other words: inequality (142) implies that Cauchy stress states including only surface contact forces cannot be generalized simply by adding contact edge forces.

Proof. Let $V$ be an admissible domain. Let its boundary $\mathcal{S}$ contain an edge $\mathcal{L}$, let $x_{0}$ be a regular point of this edge. $\mathcal{S}$ is tangent at the point $x_{0}$ to the dihedral shape $\left(n_{1}, n_{2}, \tau\right)$. In this proof we consider the case when the dihedral angle belongs to ]0, $\pi$ [ (The proof has to be slightly modified if the angle is greater than $\pi)$. We use the coordinate system $\left(x_{0} ; e_{1}, e_{2}, e_{3}\right)$ with

$$
e_{2}=\tau, \quad e_{3}=n_{1}+n_{2} /\left\|n_{1}+n_{2}\right\|
$$

For any $\varepsilon>0$, let us translate $V$ and its contact surface relatively to the vector $\varepsilon^{2} e_{3}$ :

$$
V^{\prime}:=t_{\varepsilon^{2} e_{3}}(V), \quad \mathcal{S}^{\prime}:=t_{\varepsilon^{2} e_{3}}(\mathcal{S}), \quad \mathcal{L}^{\prime}=t_{\varepsilon^{2} e_{3}}(\mathcal{L})
$$

and let us define $V_{\varepsilon}$ as the intersection of the domain $V^{\prime}$ with the parallelepiped (represented in the introduced coordinate system)

$$
P_{\varepsilon}=\left[-c \varepsilon^{2}, c \varepsilon^{2}\right] \times[0, \ell \varepsilon] \times\left[0,2 \varepsilon^{2}\right]
$$

(see Figure 2 in dell'Isola and Seppecher (1997)). The dihedral angle belongs to $] 0, \pi[$ and the curvatures of the faces of $\mathcal{S}$ and of the edge $\mathcal{L}$ are bounded. Then $c$ and $\ell$ may be chosen in such a way that, for $\varepsilon$ small enough: i) $\mathcal{L}^{\prime}$ meets $\partial P_{\varepsilon}$ on the surfaces $\left\{x . e_{2}=0\right\}$ and $\left\{x . e_{2}=\ell \varepsilon\right\}$, so that at every point $x$ on $\mathcal{L}^{\prime} \cap P_{\varepsilon}$, we have $x . e_{3}>0$ ii) $\mathcal{S}^{\prime}$ meets $\partial P_{\varepsilon}$ on surfaces $\left\{x . e_{2}=0\right\},\left\{x . e_{2}=\ell \varepsilon\right\}$ and on the surface $\left\{x . e_{3}=0\right\}$. The geometry of considered construction is illustrated by Figure 2 in dell'Isola and Seppecher (1997). We denote by $\mathcal{S}_{\varepsilon}$ the boundary of $V_{\varepsilon}$ and by $\mathcal{L}_{\varepsilon}$ the upper edge of $V_{\varepsilon}$ : in formulas

$$
\mathcal{L}_{\varepsilon}=\mathcal{L}^{\prime} \cap P_{\varepsilon}
$$


The family of shapes $\bigcup_{\varepsilon>0} \Phi\left(\mathcal{S}_{\varepsilon}\right)$ is a set of prescribed shapes or prescribed plane cuts. Then the surface force and the line force densities

$$
\hat{\mathrm{F}}_{0}^{l}\left(x_{0},\left[\left(x_{0}, \mathcal{S}_{\varepsilon}\right)\right]\right) \quad \hat{\mathrm{F}}_{0}^{s}\left(x_{0},\left[\left(x_{0}, \mathcal{S}_{\varepsilon}\right)\right]\right)
$$

are uniformly bounded with respect to $\varepsilon$. Let us consider the vector field (this vector field will be kept fixed in our limit process)

$$
U: x \mapsto\left(x . e_{3}\right) U_{0}
$$

$U_{0}$ being a generic vector. The geometry of $V_{\varepsilon}$ assures that $\varepsilon^{-2} U$ is bounded independently of $\varepsilon$. On the other hand, as $U$ vanishes on the plane $\left(x_{0}, e_{1}, e_{2}\right)$, we do not have to consider the forces exerted on the edges which are included in this plane. Considering the measure of each face and edge, we get from inequality (142)

$$
\lim _{\varepsilon \longrightarrow 0} \varepsilon^{-3} \int_{\mathcal{L}_{\varepsilon}} \hat{\mathbf{F}}_{0}^{l}\left(x,\left[\left(x, \mathcal{S}_{\varepsilon}\right)\right]\right) \cdot U(x) d l=0 .
$$

The length of $\mathcal{L}_{\varepsilon}$ is equal to $\varepsilon \ell$ within higher order terms. On the other hand, there exists a positive scalar $k$ (depending on the curvature of the edge at $x_{0}$ and on $\ell$ but independent of $\varepsilon$ ) such that

$$
\lim _{\varepsilon \longrightarrow 0} \varepsilon^{-3} \int_{\mathcal{L}_{\varepsilon}}\left(x \cdot e_{3}\right) d l=k .
$$

Let $\delta>0$, the geometry of the domain and Proposition 10.30 - which is a consequence of our regularity assumptions - imply that, for $\varepsilon$ small enough,

$$
\forall x \in \mathcal{L}_{\varepsilon},\left\|\hat{\mathrm{F}}_{0}^{l}\left(x,\left[\left(x, \mathcal{S}_{\varepsilon}\right)\right]\right)-\hat{\mathrm{F}}_{0}^{l}\left(x_{0},\left[\left(x_{0}, \mathcal{S}\right)\right]\right)\right\|<\delta .
$$

Then

$$
\left|k \hat{\mathrm{F}}_{0}^{l}\left(x_{0},\left[\left(x_{0}, \mathcal{S}\right)\right]\right) \cdot U_{0}-\lim _{\varepsilon \longrightarrow 0} \varepsilon^{-3} \int_{L_{\varepsilon}} \hat{\mathrm{F}}_{0}^{l}\left(x,\left[\left(x, \mathcal{S}_{\varepsilon}\right)\right]\right) \cdot U(x) d l\right|<k \delta\left\|U_{0}\right\| .
$$

This result holds for any $\delta$ and for any $U_{0}$, so that we can conclude with the searched equality

$$
\hat{\mathrm{F}}_{0}^{l}\left(x_{0},\left[\left(x_{0}, \mathcal{S}\right)\right]\right)=0 .
$$




\section{Generalized Noll Theorem}

Theorem 11.7 states that when stress state is of the form specified as in 140 and if one accepts the quasi-balance of contact powers 142 then there are no contact edge forces.

In this section we prove a generalization of Noll theorem for such stress states.

Remark 12.1. In Noll (1959) it is proven (we use the language introduced in the present work) that given a body i) occupying in a configuration the compact region $D$, ii) for which the stress state is characterized by the only function $\hat{\mathrm{F}}_{0}^{s}$, and iii) such that $\left\|\hat{\mathrm{F}}_{0}^{s}\right\|$ is bounded in the set $D \times \Phi^{r}$ (i.e. if contact surface forces are uniformly bounded in the set $\Phi^{r}$ of all possible regular shapes of contact surfaces!!) then for every regular shape $f$ tangent to the plane $\pi$ in the point $x$ the following equality holds

$$
\hat{\mathrm{F}}_{0}^{s}(x, f)=\hat{\mathrm{F}}_{0}^{s}(x,[(x, \pi)]) .
$$

Remark 12.2. The cited result by Noll cannot be applied in those circumstances in which one wants, e.g., to allow for a linear dependence of contact surface forces on local curvature of contact surface. In Noll and Virga (1990) it was announced for a future paper a generalization of Noll's result by relaxing the previously considered condition iii). We could not find such a paper in the literature.

Remark 12.3. The regularity assumptions for stress state proposed in dell'Isola and Seppecher (1997) and generalized in the present work actually relaxed the aforementioned condition iii) and allow for a more general dependence of stress state functions on shapes. In particular our assumptions allow for a linear dependence of surface contact forces on curvature tensor of contact surface.

Remark 12.4. In dell'Isola and Seppecher (1997) it is proved a generalization of Noll's result under the weaker regularity assumptions $\mathbf{1 0 . 1}$ on which also the present work is based.

We give now a proof of property 159 when stress state is characterized only by a surface contact force function $\hat{\mathrm{F}}_{0}^{s}$ which, however, is not bounded in the set of all regular shapes.

Theorem 12.5. When no edge forces are present, and under the regularity assumptions 10.1, for all regular shapes $f$ tangent to the plane shape $n$ the validity of quasi-balance of power 142 implies

$$
\hat{\mathrm{F}}_{0}^{s}(x, f)=\hat{\mathrm{F}}_{0}^{s}(x, n) .
$$


Proof. Let $S$ be the boundary of an admissible domain $V$ and let $x_{0}$ be a regular point of $S^{r}$. We call $n_{0}$ the normal to $S$ at $x_{0}$. We use the coordinate system $\left(x_{0}, e_{1}, e_{2}, e_{3}\right)$ (with $\left.e_{3}=n_{0}\right)$. Let us consider the family of parallelepipeds

$$
C_{\varepsilon}=[0, \varepsilon] \times[0, \varepsilon] \times\left[-c \varepsilon^{2}, c \varepsilon^{2}\right]
$$

Let us define the sets

$$
V_{\varepsilon}:=V \cap C_{\varepsilon}, \quad S_{\varepsilon}=S \cap C_{\varepsilon}
$$

As the curvature of $S$ is bounded in a neighborhood of $x_{0}$, a positive scalar $c$ can be found such that, for $\varepsilon$ sufficiently small, $S_{\varepsilon}$ does not intersect one of the faces

$$
S^{+}:=\left\{x_{3}=c \varepsilon^{2}\right\}, \quad S^{-}=\left\{x_{3}=-c \varepsilon^{2}\right\}
$$

of $C_{\varepsilon}$ (see Figure 3 in dell'Isola and Seppecher (1997)). The shapes of the boundary of $V_{\varepsilon}$ are either prescribed shapes or prescribed plane cuts. Inequality (138) when applied to $C_{\varepsilon}$ implies

$$
\lim _{\varepsilon \longrightarrow 0} \varepsilon^{-2} \int_{S^{+}} \hat{\mathrm{F}}_{0}^{s}\left(x, n_{0}\right) d s+\lim _{\varepsilon \longrightarrow 0} \varepsilon^{-2} \int_{S^{-}} \hat{\mathrm{F}}_{0}^{s}\left(x,-n_{0}\right) d s=0,
$$

which leads to

$$
\hat{\mathrm{F}}_{0}^{s}\left(x, n_{0}\right)=-\hat{\mathrm{F}}_{0}^{s}\left(x,-n_{0}\right) .
$$

Inequality (138) when applied to $V_{\varepsilon}$ implies

$$
\lim _{\varepsilon \longrightarrow 0} \varepsilon^{-2} \int_{S_{\varepsilon}} \hat{\mathrm{F}}_{0}^{s}(x,[(x, S)]) d s+\lim _{\varepsilon \longrightarrow 0} \varepsilon^{-2} \int_{S^{-}} \hat{\mathrm{F}}_{0}^{s}\left(x,-n_{0}\right) d s=0
$$

which leads to

$$
\hat{\mathrm{F}}_{0}^{s}(x,[(x, S)])+\hat{\mathrm{F}}_{0}^{s}\left(x,-n_{0}\right)=0 .
$$

Equation (160) is then obtained when recalling (162).

Remark 12.6. In the previous proof we just had to modify the argument used in Truesdell (1977) by using a cylinder whose basis is a square instead of a circle. The important difference is that -in this way- we use only prescribed shapes (in the sense defined in ??). 


\subsection{CAUCHY THEOREM}

Stress states verifying the hypotheses of Theorem 11.7 cannot include nonvanishing contact edge forces. Then the Cauchy's construction of stress tensor, which is strongly depending on the absence of edge contact forces, (refer to Truesdell (1977)) is valid.

Theorem 12.7. When stress state is of the form specified in 140 and if one accepts regularity assumptions 10.1 and the quasi-balance of contact powers 142 there exists a continuous tensor field $T$ of order two and symmetric such that, for any plane shape $n$,

$$
\hat{\mathrm{F}}_{0}^{s}(x, n)=T(x) . n .
$$

\section{The structure imposed on stress states by the regularity assumptions 10.1 and quasi-balance of contact power}

In this section we show how to generalize the results found in dell'Isola and Seppecher (1995)-(1997) to $N-t h$ gradient continua. The complete proof strategy is simply drafted: all the details of the mathematical concepts and proofs will be presented in a forthcoming separate paper.

We start with a "rough" statement which will be followed immediately by a more formal one.

Balance of power implies that the stress state in a $n-t h$ order generalized continuum $B$ must verify the following conditions

1. The existence of $k$-forces concentrated on wedges implies the existence of line $k+1$-forces distributed on edges

2. The existence of lines $k+1$-forces distributed on edges implies the existence of surfaces $k+2$-forces distributed on faces

3 . The existence of surfaces $k+2$-forces distributed on faces implies the existence of $n=k+3$-stresses in the volume occupied by the body.

More formally after the following

Remark 13.1. When considering a stress state, instead of using the previously introduced notation

$$
\left\{\mathrm{F}_{k}^{s}, \mathrm{~F}_{k}^{l}, \mathrm{~F}_{k}^{c} ; k=0, \ldots, L\right\},
$$

we will use another one slightly different. Indeed: instead of the superscripts $s, l,{ }^{c}$ we want to use the superscripts ${ }^{2},{ }^{1},{ }^{0}$. 
This change of notation will be useful in the formulation of the following definitions and theorem.

Definition 13.2. Let $B$ a body occupying in one of its configurations the domain $D$. Let us consider a stress state $\mathfrak{S}$ of the form

$$
\mathfrak{S}=\left\{\mathrm{F}_{k}^{2}, \mathrm{~F}_{k}^{1}, \mathrm{~F}_{k}^{0} ; k=0, \ldots, L\right\} .
$$

where all $\mathrm{F}$ are defined in $D$. Let $\mathcal{S}$ be the contact surface of $D$, and let $\mathcal{S}_{r}$, $\mathcal{L}_{r}$ and $\mathcal{W}_{S}$ denote the subsets of $\mathcal{S}$ constituted by regular surface and edge points and wedges respectively. The power expended on the velocity field $U$ by contact actions exerted on $D$ through $\mathcal{S}$ (when $B$ is in the stress state $\mathfrak{S})$ is given by the following formula:

$$
\begin{aligned}
\mathcal{P}_{U}(\mathfrak{S}, D) & :=\left(\int_{\mathcal{S}_{r}} \mathrm{~F}_{0}^{2} \cdot U+\int_{\mathcal{L}_{r}} \mathrm{~F}_{0}^{1} \cdot U+\int_{\mathcal{W}_{S}} \mathrm{~F}_{0}^{0} \cdot U\right)+ \\
& +\sum_{k=1}^{L}\left(\int_{\mathcal{S}_{r}} \mathrm{~F}_{k}^{2} \cdot\left(\nabla^{k} U\right)_{\perp}+\int_{\mathcal{L}_{r}} \mathrm{~F}_{k}^{1} \cdot\left(\nabla^{k} U\right)_{\perp}+\int_{\mathcal{W}_{S}} \mathrm{~F}_{k}^{0} \cdot \nabla^{k} U\right)
\end{aligned}
$$

When this will not lead to confusion we will skip the argument $\mathfrak{S}$ in the previous expression.

Definition 13.3. Let us consider a stress state $\mathfrak{S}$.We say that it is physically admissible when it verifies i) the regularity assumptions 10.1 and ii) the quasi-balance of power

$$
\left|\mathcal{P}_{U}(\mathfrak{S}, D)\right| \leq K_{U}|D|
$$

Theorem 13.4. Let us consider a stress state $\mathfrak{S}$ of the form

$$
\mathfrak{S}=\left\{\mathrm{F}_{k}^{2}, \mathrm{~F}_{k}^{1}, \mathrm{~F}_{k}^{0} ; k=0, \ldots, L\right\} .
$$

If $\mathfrak{S}$ is physically admissible then there exists a (unique) natural number $N-1 \leq L$ such that

$$
\begin{gathered}
\mathrm{F}_{N-1}^{2} \neq 0 \\
(\forall k \in\{0, \ldots, L\})(\forall a \in\{0,1,2\})\left((k+2 \geq N+a) \Rightarrow\left(\mathrm{F}_{k}^{a}=0\right)\right)
\end{gathered}
$$

Proof. It can be sketched by paralleling the previous proof of Theorem 11.7 .

Definition 13.5. Let $\mathfrak{S}$ be a physically admissible stress state. We call grade of $\mathfrak{S}$ the unique natural number $N_{\mathfrak{S}}$ the existence of which has been proven in the previous theorem. 
It can also be proven the following:

Theorem 13.6. Let $\mathfrak{S}$ be a physically admissible stress state of grade $N$. If $\mathrm{F}_{N-2}^{1}=0$ then $\mathrm{F}_{N-3}^{0}=0$.

Proof. The searched implication is obtained by means of a construction generalizing the one used for Theorem 11.7.

Definition 13.7. Let $B$ be a body. We say that $B$ is a $N-t h$ gradient continuum if i) the set of the stress states admissible for $B$ is included in the set of physically admissible stresses of grade $N$, and ii) there exists at least one nonvanishing admissible stress state for $B$ the grade of which is exactly $N$.

\section{The tetrahedron argument applied to N-th gradient continua}

In this final section we draft a line of thought which proves how the tetrahedron argument valid for second gradient materials which was presented in dell'Isola and Seppecher (1995),(1997) actually can be extended to encompass all those continua imagined by Green and Rivlin (1964) and described in the previous sections.

\subsection{On contact actions including forces of order greater than one.}

Theorems 13.4 and 13.6 have shown that not all stress states are physically admissible. In the present subsection we want to establish a characterization theorem, which states how to construct physically admissible stress states by means of suitably introduced (hyper-)stress tensor fields. We start with second gradient continua.

\section{Surface Double Forces}

In dell'Isola and Seppecher (1995)-(1997) it was proven the impossibility -under the assumption (142)- of considering stress states including only nonvanishing surface and contact edge forces. The idea developed there was the following: the power of contact actions is actually quasi-balanced but the expression for this power used in inequality (142) is too naive. Indeed the contact actions must be endowed with a more complex structure: to 
be precise (using the presently used notation) the simplest stress states which can be considered -after those studied by Cauchy- have the following structure (typical of Germain's second gradient continua)

$$
\mathfrak{S}=\left\{\mathrm{F}_{0}^{2}, \mathrm{~F}_{1}^{2}, \mathrm{~F}_{0}^{1}\right\} .
$$

Remark 14.1. From a physical point of view, the need of introducing a contact double force or contact '1-normal force' can be justified as follows: in the balance of energy an additional term must be considered which does not appear in the balance of forces. An alternative approach trying to meet this need is due to Dunn and Serrin (1985), who introduced directly a supplementary flux of energy ('interstitial working'). Our approach, based on the concept of Schwartz distributions, has the following advantages when compared with that found there: (i) it does not assume a priori that the extra energetic term is a flux, (ii) it shows the mechanical nature of this term, its linear dependence on the velocity field being a basic assumption, (iii) it naturally yields general and physically meaningful boundary conditions (see e.g. Seppecher (1989)).

Most mechanicians will not be surprised by the introduction of contact double forces distributions, as contact couples are needed already in the standard theories of beams and shells. Another example of '1-normal' contact forces distribution found in the literature (this time for 3-D continua) is given by couple stresses introduced by Cosserat (1908)-(1909). The microscopic meaning of contact forces of order greater than one can be understood by considering the asymptotic limit of non-local short range interactions.

Example 14.2. A system of non-local short range forces converging to a surface contact double force (1-normal distribution). Using the Cartesian coordinates $\left(x_{1} ; x_{2} ; x_{3}\right)$, the domain

$$
V=\left\{x: x_{1}<0\right\}
$$

is in contact with the external world through the plane

$$
S=\left\{x: x_{1}=0\right\} .
$$

Assume that the external forces exerted on $V$ have short range $\varepsilon \ll 1$ (compared with some other characteristic length) and that these forces are represented by the volume density

$$
f_{\varepsilon}(x)=f_{0}^{-\gamma} \varphi\left(\varepsilon^{-1} x_{1}\right)
$$

where $\varphi$ is a function whose support is a compact set included in ]- -0[$ and $f_{0}$ is a given vector. If $\gamma=1$ and $\varphi$ is a non negative function whose 
integral is equal to 1 , the distribution tends, as $\varepsilon$ tends to 0 , to the vector measure on $S$ having a surface density equal to $f_{0}$ : this is the classical case of surface force density. However, if $\gamma=2$ and $\varphi$ is the derivative of a non negative function whose integral is equal to 1 , the distribution tends to a '1-normal' distribution $D$ such that

$$
D(U)=\int_{S} f_{0} \cdot \partial U / \partial x_{1} d s .
$$

This force distribution i) is localized on $S$, ii) has no influence upon balance of forces, iii) supplies energy in presence of velocity fields in $V$, even when these fields vanish on $S$.

The assumption of quasi-balance of contact actions for stress states of the form 173 takes the following particular form:

for all $C^{\infty}$ field $U$, there exists a scalar $K_{U}$ such that, for any admissible domain $V$ having contact surface $S$ with surface and edge regular point sets denoted respectively by $S_{r}$ and $L_{r}$, we have

$$
\left|\begin{array}{c}
\int_{S^{r}} \mathrm{~F}_{1}^{2}(x,[(x, S)]) \cdot \frac{\partial U}{\partial n}(x) d s+\int_{S^{r}} \mathrm{~F}_{0}^{2}(x,[(x, S)]) \cdot U(x) d s \\
+\int_{L^{r}} \mathrm{~F}_{0}^{1}(x,[(x, S)]) \cdot U(x) d l
\end{array}\right|<K_{U}|V| .
$$

Remark 14.3. This assumption is less stringent on $F_{0}^{1}$ than the corresponding hypothesis (142). It will imply less stringent restrictions upon edge contact forces. Indeed condition (174) does not imply that edge contact forces must be vanishing.

Remark 14.4. The fact that (174) implies the quasi-balance of forces (138) is still true (it can be again verified by considering three linearly independent constant fields $U$ ). Again, we do not need any assumption on the behavior of $K_{U}$ with respect to $U$.

Remark 14.5. Note that, in each proof we present, a limit in inequality (174) is calculated with a fixed field $U$. For this reason we do not need any assumption on the behavior of $K_{U}$ with respect to $U$.

\section{Dependence of second order stress functions on the shape of the contact surface. A theorem analogous to Noll Theorem.}

We first prove a version of action-reaction principle valid for double forces i.e. for second order stress functions. 
The theorem, at this stage, concerns only plane shapes.

Theorem 14.6. Under regularity assumptions 10.1 and having postulated the quasi-balance of contact actions, at every point $x$, for all plane shape $n$ and for all dihedral shape $\left(n, e_{1}, e_{2}\right)$ we have

$$
\begin{gathered}
\mathrm{F}_{1}^{2}(x, n)-\mathrm{F}_{1}^{2}(x,-n)=0 . \\
\mathrm{F}_{0}^{1}\left(x_{0},\left(n,-e_{1}, e_{2}\right)\right)+\mathrm{F}_{0}^{1}\left(x_{0},\left(n, e_{1}, e_{2}\right)\right)=0 ; \\
\mathrm{F}_{0}^{1}\left(x_{0},\left(-n, e_{1}, e_{2}\right)\right)+\mathrm{F}_{0}^{1}\left(x_{0},\left(n, e_{1}, e_{2}\right)\right)=0 ; \\
\mathrm{F}_{0}^{1}\left(x_{0},\left(n, e_{1},-e_{2}\right)\right)+\mathrm{F}_{0}^{1}\left(x_{0},\left(n, e_{1}, e_{2}\right)\right)=0
\end{gathered}
$$

Proof. Using the coordinate system $\left(x_{0}, e_{1}, e_{2}, e_{3}\right)$ (with $e_{3}=n$ : the vectors $e_{i}$ are unitary, but not necessarily orthogonal), let introduce for every point its coordinates $\left(x_{1}, x_{2}, x_{3}\right)$ in the introduced system and let us consider the domain

$$
C_{\varepsilon}=\left[0, \varepsilon^{\alpha}\right] \times\left[0, \varepsilon^{\beta}\right] \times\left[0, \varepsilon^{\gamma}\right]
$$

(where $\alpha, \beta$ and $\gamma$ are positive) and the vector field

$$
\bar{U}: x \mapsto x_{3} U_{0}
$$

where $U_{0}$ is a given vector. We consider each face or edge of $\partial C_{\varepsilon}$ on which some addends of LHS in inequality (174) when calculated on the velocity field 177 are nonvanishing i.e.

$$
\begin{aligned}
& S_{\varepsilon}^{+}:=\left\{x: x_{3}=+\varepsilon^{\gamma}\right\}, \quad S_{\varepsilon}^{-}:=\left\{x: x_{3}=0\right\} \\
& L_{\alpha 0}^{+}:=\left[0, \varepsilon^{\alpha}\right] \times\{0\} \times\left\{\varepsilon^{\gamma}\right\} \quad L_{\alpha \beta}^{+}:=\left[0, \varepsilon^{\alpha}\right] \times\left\{\varepsilon^{\beta}\right\} \times\left\{\varepsilon^{\gamma}\right\} \\
& L_{\beta 0}^{+}:=\{0\} \times\left[0, \varepsilon^{\beta}\right] \times\left\{\varepsilon^{\gamma}\right\} \quad L_{\beta \alpha}^{+}:=\left\{\varepsilon^{\alpha}\right\} \times\left[0, \varepsilon^{\beta}\right] \times\left\{\varepsilon^{\gamma}\right\} \\
& L_{00}:=\{(0,0)\} \times\left[0, \varepsilon^{\gamma}\right] \quad L_{0 \beta}:=\left\{\left(0, \varepsilon^{\beta}\right)\right\} \times\left[0, \varepsilon^{\gamma}\right] \\
& L_{\alpha 0}:=\left\{\left(\varepsilon^{\alpha}, 0\right)\right\} \times\left[0, \varepsilon^{\gamma}\right] \quad L_{\alpha \beta}^{\vec{\alpha}}:=\left\{\left(\varepsilon^{\alpha}, \varepsilon^{\beta}\right)\right\} \times\left[0, \varepsilon^{\gamma}\right]
\end{aligned}
$$

the area or length of which are respectively given by

$$
\begin{gathered}
\mu_{2}\left(S_{\varepsilon}^{+}\right)=\mu_{2}\left(S_{\varepsilon}^{-}\right)=\varepsilon^{\alpha \beta} ; \quad \mu_{1}\left(L_{\alpha 0}^{+}\right)=\mu_{1}\left(L_{\alpha \beta}^{+}\right)=\varepsilon^{\alpha} ; \\
\mu_{1}\left(L_{\beta 0}^{+}\right)=\mu_{1}\left(L_{\beta \alpha}^{+}\right)=\varepsilon^{\beta} ; \\
\mu_{1}\left(L_{00}\right)=\mu_{1}\left(L_{0 \beta}\right)=\mu_{1}\left(L_{\alpha 0}\right)=\mu_{1}\left(L_{\alpha \beta}\right)=\varepsilon^{\gamma} .
\end{gathered}
$$

As

$$
\begin{aligned}
& \left(\forall x \in S_{\varepsilon}^{+}\right)\left(x_{3}=\varepsilon^{\gamma}\right) ; \\
& \left(\forall x \in S_{\varepsilon}^{-}\right)\left(x_{3}=0\right) ; \\
& \left(\forall x \in S_{\varepsilon}^{ \pm}\right)\left(\partial \bar{U} / \partial n= \pm U_{0}\right)
\end{aligned}
$$


and $\mu_{3}\left(C_{\varepsilon}\right)=\varepsilon^{\alpha} \varepsilon^{\beta} \varepsilon^{\gamma}$; the inequality (174) applied to $C_{\varepsilon}$ and the field 177 becomes:

$$
\begin{gathered}
\mid \int_{S_{\varepsilon}^{+}} \mathrm{F}_{1}^{2}(x, n) \cdot U_{0} d s+\int_{S_{\varepsilon}^{-}} \mathrm{F}_{1}^{2}(x,-n) \cdot\left(-U_{0}\right) d s+ \\
+\int_{S_{\varepsilon}^{+}} \mathrm{F}_{0}^{2}(x, n) \cdot \varepsilon^{\gamma} U_{0} d s+\int_{L_{\alpha}^{+}} \mathrm{F}_{0}^{1}\left(x,\left[\left(x, \partial C_{\varepsilon}\right)\right]\right) \cdot \varepsilon^{\gamma} U_{0} d l+ \\
\quad+\int_{L_{\beta}^{+}} \mathrm{F}_{0}^{1}\left(x,\left[\left(x, \partial C_{\varepsilon}\right)\right]\right) \cdot \varepsilon^{\gamma} U_{0} d l+ \\
+\int_{L_{\vec{\varepsilon}}} \mathrm{F}_{0}^{1}\left(x,\left[\left(x, \partial C_{\varepsilon}\right)\right]\right) \cdot\left(\left(x-x_{0}\right) \cdot n\right) U_{0} d l \mid<K_{\bar{U}}\left(\varepsilon^{\alpha} \varepsilon^{\beta} \varepsilon^{\gamma}\right) .
\end{gathered}
$$

where

$$
L_{\alpha}^{+}=L_{\alpha 0}^{+} \cup L_{\alpha \beta}^{+} ; \quad L_{\beta}^{+}=L_{\beta 0}^{+} \cup L_{\beta \alpha}^{+} ; \quad L_{\varepsilon}^{\rightarrow}=L_{00} \cup L_{0 \beta} \cup L_{\alpha 0} \cup L_{\alpha \beta}
$$

Remarking that

$$
\begin{gathered}
x \in L^{+} \Rightarrow[(x, S)]=\left(e_{1}, n, n \times e_{1}\right) \\
\text { or }\left(e_{2}, n, n \times e_{2}\right) \text { or }\left(-e_{1}, n,-n \times e_{1}\right) \text { or }\left(-e_{2}, n,-n \times e_{2}\right) \\
x \in L_{\varepsilon}^{\rightarrow} \Rightarrow[(x, S)]=\left(-e_{2},-e_{1}, n\right) \\
\text { or }\left(e_{2},-e_{1}, n\right) \text { or }\left(e_{2}, e_{1}, n\right) \text { or }\left(e_{1},-e_{2}, n\right)
\end{gathered}
$$

a simple inspection of previous formulas allows us to recognize that $\Phi\left(\partial C_{\varepsilon}\right)$ is a set of prescribed shapes and therefore that all $F$ are uniformly bounded on the set

$$
\bigcup_{\varepsilon>0}\left(C_{\varepsilon} \times \Phi\left(\partial C_{\varepsilon}\right)\right)
$$

As a consequence we can state that there exist suitable constants $K$ such 
that

$$
\begin{gathered}
\left|\int_{S_{\varepsilon}^{ \pm}} \mathrm{F}_{1}^{2}(x, n) \cdot U_{0} d s\right|<K_{1}^{2}\left\|U_{0}\right\| \varepsilon^{\alpha} \varepsilon^{\beta} \\
\left|\int_{S_{\varepsilon}^{+}} \mathrm{F}_{0}^{2}(x, n) \cdot \varepsilon^{\gamma} U_{0} d s\right|<K_{0}^{2}\left\|U_{0}\right\| \varepsilon^{\alpha} \varepsilon^{\beta} \varepsilon^{\gamma} \\
\left|\int_{L_{\alpha}^{+}} \mathrm{F}_{0}^{1}\left(x,\left[\left(x, \partial C_{\varepsilon}\right)\right]\right) \cdot \varepsilon^{\gamma} U_{0} d l\right|<K_{0}^{1}\left\|U_{0}\right\| \varepsilon^{\alpha} \varepsilon^{\gamma} \\
\left|\int_{L_{\beta}^{+}} \mathrm{F}_{0}^{1}\left(x,\left[\left(x, \partial C_{\varepsilon}\right)\right]\right) \cdot \varepsilon^{\gamma} U_{0} d l\right|<K_{0}^{1}\left\|U_{0}\right\| \varepsilon^{\beta} \varepsilon^{\gamma} \\
\left|\int_{L_{\vec{\varepsilon}}} \mathrm{F}_{0}^{1}\left(x,\left[\left(x, \partial C_{\varepsilon}\right)\right]\right) \cdot\left(\left(x-x_{0}\right) \cdot n\right) U_{0} d l\right|<K_{0}^{1}\left\|U_{0}\right\| \varepsilon^{2 \gamma} .
\end{gathered}
$$

If one chooses $\gamma>\beta$ and $\gamma>\alpha$ the inequality 187 (multiplied times $\varepsilon^{-\alpha} \varepsilon^{-\beta}$ ) implies that

$$
\lim _{\varepsilon \rightarrow 0} \varepsilon^{-\alpha} \varepsilon^{-\beta}\left(\int_{S_{\varepsilon}^{+}} F_{1}^{2}(x, n) \cdot U_{0} d s+\int_{S_{\varepsilon}^{-}} F_{1}^{2}(x,-n) .\left(-U_{0}\right) d s\right)=0
$$

The continuity properties of the partial function $\mathrm{F}_{1}^{2}(\cdot, n)$ and the arbitrariness of $U_{0}$ finally imply

$$
\mathrm{F}_{1}^{2}\left(x_{0}, n\right)=\mathrm{F}_{1}^{2}\left(x_{0},-n\right) .
$$

On the other hand let us multiply 187 times $\varepsilon^{-\beta} \varepsilon^{-\gamma}$, let us choose arbitrarily $\beta>0$ and consequently $\alpha$ and $\gamma$ verifying the conditions:

$$
\alpha>\gamma \quad \alpha>\beta>0 \quad(\beta>0) \wedge\left(\gamma^{2}-\beta-\gamma\right)>0 \Leftrightarrow \gamma>\frac{1}{2} \sqrt{4 \beta+1}+\frac{1}{2}
$$

and finally calculate the limit for $\varepsilon \rightarrow 0$, of the LHS of obtained inequality. Recalling 188 we get

$$
\begin{gathered}
\lim _{\varepsilon \rightarrow 0} \varepsilon^{-\beta} \varepsilon^{-\gamma}\left(\int_{L_{\beta}^{+}=L_{\beta 0}^{+} \cup L_{\beta \alpha}^{+}} \mathrm{F}_{0}^{1}\left(x,\left[\left(x, \partial C_{\varepsilon}\right)\right]\right) \cdot \varepsilon^{\gamma} U_{0} d l\right)= \\
=\lim _{\varepsilon \rightarrow 0} \varepsilon^{-\beta}\left(\int_{L_{\beta 0}^{+}} \mathrm{F}_{0}^{1}\left(x,\left(n,-e_{1}, e_{2}\right)\right) \cdot U_{0} d l+\int_{L_{\beta \alpha}^{+}} \mathrm{F}_{0}^{1}\left(x,\left(n, e_{1}, e_{2}\right)\right) \cdot U_{0} d l\right)=0
\end{gathered}
$$

The continuity properties of the partial function $\mathrm{F}_{0}^{1}(\cdot,(u, v, w))$ (where $(u, v, w)$ is a given dihedral shape) and the arbitrariness of $U_{0}$ finally imply

$$
\mathrm{F}_{0}^{1}\left(x_{0},\left(n,-e_{1}, e_{2}\right)\right)+\mathrm{F}_{0}^{1}\left(x_{0},\left(n, e_{1}, e_{2}\right)\right)=0
$$


The other two relations in 176 are obtained with similar constructions.

We are now able to prove a theorem for '1-normal' distributions analogous to the theorem of Noll (?), (?).

Theorem 14.7. Under regularity assumptions 10.1 and having postulated the quasi-balance of contact actions, at every point $x$ and for every regular shape $f$ tangent to the plane shape $n$ we have

$$
\mathrm{F}_{1}^{2}(x, f)=\mathrm{F}_{1}^{2}(x, n)
$$

that is $\mathrm{F}_{1}^{2}$, depends on the shape of the contact surface only through its normal.

Proof. The proof is close to that we have given for the Theorem 12.5. At a regular point $x_{0}$ of the boundary $S$ of an admissible domain, we consider the family of domains $V_{\varepsilon}$ described in the proof of the Theorem 12.5 (see also Figure 3 in dell'Isola and Seppecher (1997)). We consider the vector field

$$
U: x \longrightarrow\left(x \cdot n_{0}\right) U_{0}
$$

where $n_{0}$ denotes the normal to $S$ at $x_{0}$ and $U_{0}$ is any vector. The shapes of $\partial V_{\varepsilon}$ are either prescribed shapes or prescribed plane cuts. Moreover $\varepsilon^{-2} U$ is bounded in $V_{\varepsilon}$ independently of $\varepsilon$. The inequality (174) applied to $V_{\varepsilon}$ leads to

$$
\begin{aligned}
& \lim _{\varepsilon \longrightarrow 0} \varepsilon^{-2} \int_{S \cap C_{\varepsilon}} F_{1}^{2}\left(x,[(x, S)] \cdot U_{0}\left(n \cdot n_{0}\right) d s+\right. \\
& +\lim _{\varepsilon \longrightarrow 0} \varepsilon^{-2} \int_{S^{-}} F_{1}^{2}\left(x,-n_{0}\right) \cdot\left(-U_{0}\right) d s=0 .
\end{aligned}
$$

As $n \cdot n_{0}$ is a continuous function with respect to $x$ on $S$, the regularity properties of $\mathrm{F}_{1}^{2}$ and the arbitrariness of $U_{0}$ imply

$$
\mathrm{F}_{1}^{2}\left(x_{0},\left[\left(x_{0}, S\right)\right]=\mathrm{F}_{1}^{2}\left(x_{0},-n_{0}\right)=\mathrm{F}_{1}^{2}\left(x_{0}, n_{0}\right) .\right.
$$

\subsection{A Representation Theorem for surface 1-forces generaliz- ing the Cauchy tetrahedron Theorem.}

The celebrated Cauchy tetrahedron argument can be used also to get a representation Theorem which is valid for $N-t h$ gradient continua. Obviously it needs to be suitably adapted. 
The reader should not believe that the argument has to be applied, in the framework of the theory of $N-t h$ gradient continua, to the surface density of 1 - force. Instead it has to be applied to surface density of $N$-forces. It happens that $N$-force surface density depends only on the tangent planar shape to the contact surface and this dependence is a polynomial one: its grade is exactly $N$.

Theorem 14.8. Let $B$ a body occupying in one of its configurations the domain D. Let us consider a stress state $\mathfrak{S}$ having the form $\left\{\mathrm{F}_{0}^{2}, \mathrm{~F}_{1}^{2}, \mathrm{~F}_{0}^{1}\right\}$. Let us assume the regularity assumptions 10.1. If quasi balance of power holds then there exists a continuous three-tensor field $C$ such that, at any point $x_{0} \in D$ and for any plane shape $n$,

$$
\mathrm{F}_{1}^{2}\left(x_{0}, n\right)=\left(C\left(x_{0}\right) \cdot n\right) \cdot n .
$$

Proof. We follow the tetrahedron construction due to Cauchy (see Figure 4 in dell'Isola and Seppecher (1997)). In an orthonormal coordinate system $\left(x_{0}, e_{1}, e_{2}, e_{3}\right)$, we define the tetrahedron $V$ whose faces $S, S_{1}, S_{2}$ and $S_{3}$ are respectively normal to $n,-e_{1},-e_{2}$ and $-e_{3}$ and whose height (perpendicular to $S$ ) is $h$. In our construction the origin of the coordinate system belongs to the face $S$. We denote respectively by $f_{1}=\left(-e_{2},-e_{3}, e_{1}\right), f_{2}=$ $\left(-e_{3},-e_{1}, e_{2}\right)$ and $f_{3}=\left(-e_{1},-e_{2}, e_{3}\right)$ the shapes of the edges $L_{1}, L_{2}$ and $L_{3}$. Let $V_{\varepsilon}$ be the image of $V$ under an homothetic transformation of ratio $\varepsilon$, we denote by $S_{\varepsilon}, S_{i \varepsilon}$ and $L_{i \varepsilon}$, and the faces and edges images of $S, S_{i}$ and $L_{i}(i=1,2,3)$ under this transformation. We consider the field $U: x \longrightarrow(x \cdot n) U_{0}$, where $U_{0}$ is any vector. As this field vanishes on $S_{\varepsilon}$, the inequality (174) applied to the domain $V_{\varepsilon}$ implies

$$
\begin{aligned}
\mid \sum_{i=1}^{3}\left\{\int_{S_{i \varepsilon}}(x \cdot n) \mathrm{F}_{0}^{2}\left(x,-e_{i}\right) \cdot U_{0} d s\right\}+ \\
+\sum_{i=1}^{3}\left\{\int_{L_{i \varepsilon}}(x \cdot n) \mathrm{F}_{0}^{1}\left(x, f_{i}\right) \cdot U_{0} d l\right\}+ \\
+\sum_{i=1}^{3}\left\{\int_{S_{i \varepsilon}}\left(-e_{i} \cdot n\right) \mathrm{F}_{1}^{2}\left(x,-e_{i}\right) \cdot U_{0} d s\right\}+ \\
+\int_{S_{\varepsilon}} \mathrm{F}_{1}^{2}(x, n) \cdot U_{0} d s \mid<K \varepsilon^{3} .
\end{aligned}
$$

Let us multiply this inequality by $\varepsilon^{-2}$ and, changing variables in the integrals in order to transform them into integrals on the boundary of $V$, we 
obtain

$$
\begin{gathered}
\mid \varepsilon \sum_{i=1}^{3}\left\{\int_{S_{i}}(x \cdot n) \mathrm{F}_{0}^{2}\left(\varepsilon x,-e_{i}\right) \cdot U_{0} d s\right\} \\
+\sum_{i=1}^{3}\left\{\int_{L_{i}}(x \cdot n) \mathrm{F}_{0}^{1}\left(\varepsilon x, f_{i}\right) \cdot U_{0} d l\right\} \\
+\sum_{i=1}^{3}\left\{\int_{S_{i}}\left(-e_{i} \cdot n\right) \mathrm{F}_{1}^{2}\left(\varepsilon x,-e_{i}\right) \cdot U_{0} d s\right\}+\int_{S} \mathrm{~F}_{1}^{2}(\varepsilon x, n) \cdot U_{0} d s \mid<K \varepsilon .
\end{gathered}
$$

As the partial functions $\mathrm{F}(\cdot, f)$ are continuous, evaluating the limit as $\varepsilon$ tends to 0 , we get

$$
\begin{gathered}
\sum_{i=1}^{3}\left\{\mathrm{~F}_{0}^{1}\left(x_{0}, f_{i}\right) \cdot U_{0} \int_{L_{i}}(x \cdot n) d l\right\}+ \\
+\sum_{i=1}^{3}\left\{\left|S_{i}\right|\left(-e_{i} \cdot n\right) \mathrm{F}_{1}^{2}\left(x_{0},-e_{i}\right) \cdot U_{0}\right\}+ \\
+|S| \mathrm{F}_{1}^{2}\left(x_{0}, n\right) \cdot U_{0}=0 .
\end{gathered}
$$

This being valid for any $U_{0}$, we obtain

$$
\begin{gathered}
2|S| \mathrm{F}_{1}^{2}\left(x_{0}, n\right)=\sum_{i=1}^{3}\left\{\mathrm{~F}_{0}^{1}\left(x_{0}, f_{i}\right)\left(n \cdot e^{i}\right)\left|L_{i}\right|^{2}\right\}+ \\
+2 \sum_{i=1}^{3}\left\{\mathrm{~F}_{1}^{2}\left(x_{0},-e_{i}\right)\left|S_{i}\right|\left(n \cdot e_{i}\right)\right\} .
\end{gathered}
$$

Using the geometrical relations

$$
\begin{gathered}
h=\left|L_{1}\right|\left(n \cdot e_{1}\right)=\left|L_{2}\right|\left(n \cdot e_{2}\right)=\left|L_{3}\right|\left(n \cdot e_{3}\right), \\
2|S| h=2\left|S_{1}\right|\left|L_{1}\right|=2\left|S_{2}\right|\left|L_{2}\right|=2\left|S_{3}\right|\left|L_{3}\right|=\left|L_{1}\right|\left|L_{2}\right|\left|L_{3}\right|
\end{gathered}
$$

and Theorem 14.6, we get

$$
\begin{gathered}
\mathrm{F}_{1}^{2}\left(x_{0}, n\right)=\mathrm{F}_{0}^{1}\left(x_{0}, f_{1}\right)\left(n \cdot e_{2}\right)\left(n \cdot e_{3}\right)+\mathrm{F}_{0}^{1}\left(x_{0}, f_{2}\right)\left(n \cdot e_{3}\right)\left(n \cdot e_{1}\right)+ \\
+\mathrm{F}_{0}^{1}\left(x_{0}, f_{3}\right)\left(n \cdot e_{1}\right)\left(n \cdot e_{2}\right)+\sum_{i=1}^{3} \mathrm{~F}_{1}^{2}\left(x_{0}, e_{i}\right)\left(n \cdot e_{i}\right)^{2} .
\end{gathered}
$$

Thus we are led to define a three-tensor field $C$ such that

$$
\mathrm{F}_{1}^{2}\left(x_{0}, n\right)=\left(C\left(x_{0}\right) . n\right) . n=C\left(x_{0}\right) . . n \otimes n
$$


This tensor is not uniquely determined, as only its right-side products by symmetric two tensors are determined. We may impose its right side symmetry, by setting

$$
\begin{gathered}
C(x)=\frac{1}{2} \mathrm{~F}_{0}^{1}\left(x, f_{1}\right) \otimes\left(e_{2} \otimes e_{3}+e_{3} \otimes e_{2}\right)+ \\
+\frac{1}{2} \mathrm{~F}_{0}^{1}\left(x, f_{2}\right) \otimes\left(e_{3} \otimes e_{1}+e_{1} \otimes e_{3}\right)+ \\
+\frac{1}{2} \mathrm{~F}_{0}^{1}\left(x, f_{3}\right) \otimes\left(e_{1} \otimes e_{2}+e_{2} \otimes e_{1}\right)+ \\
\quad+\sum_{i=1}^{3}\left\{\mathrm{~F}_{1}^{2}\left(x, e_{i}\right) \otimes e_{i} \otimes e_{i}\right\}
\end{gathered}
$$

or its left side symmetry, by setting

$$
\begin{gathered}
C(x)=\frac{1}{2} \mathrm{~F}_{0}^{1}\left(x, f_{1}\right) \otimes\left(e_{2} \otimes e_{3}+e_{3} \otimes e_{2}\right)+ \\
-\frac{1}{2}\left(e_{2} \otimes e_{3}+e_{3} \otimes e_{2}\right) \otimes \mathrm{F}_{0}^{1}\left(x, f_{1}\right)+ \\
+\frac{1}{2}\left(e_{2} \otimes \mathrm{F}_{0}^{1}\left(x, f_{1}\right) \otimes e_{3}+e_{3} \otimes \mathrm{F}_{0}^{1}\left(x, f_{1}\right) \otimes e_{2}\right)+ \\
+\frac{1}{2} \mathrm{~F}_{0}^{1}\left(x, f_{2}\right)\left(e_{3} \otimes e_{1}+e_{1} \otimes e_{3}\right)+ \\
-\frac{1}{2}\left(e_{3} \otimes e_{1}+e_{1} \otimes e_{3}\right) \otimes \mathrm{F}_{0}^{1}\left(x, f_{2}\right)+ \\
+\frac{1}{2}\left(e_{3} \otimes \mathrm{F}_{0}^{1}\left(x, f_{2}\right) \otimes e_{1}+e_{1} \otimes \mathrm{F}_{0}^{1}\left(x, f_{2}\right) \otimes e_{3}\right)+ \\
+\frac{1}{2} \mathrm{~F}_{0}^{1}\left(x, f_{3}\right)\left(e_{1} \otimes e_{2}+e_{2} \otimes e_{1}\right)+ \\
-\frac{1}{2}\left(e_{1} \otimes e_{2}+e_{2} \otimes e_{1}\right) \otimes \mathrm{F}_{0}^{1}\left(x, f_{3}\right)+ \\
+\frac{1}{2}\left(e_{1} \otimes \mathrm{F}_{0}^{1}\left(x, f_{3}\right) \otimes e_{2}+e_{2} \otimes \mathrm{F}_{0}^{1}\left(x, f_{3}\right) \otimes e_{1}\right)+ \\
+\sum_{i=1}^{3}\left\{\mathrm{~F}_{1}^{2}\left(x, e_{i}\right) \otimes e_{i} \otimes e_{i}-e_{i} \otimes e_{i} \otimes \mathrm{F}_{1}^{2}\left(x, e_{i}\right)+e_{i} \otimes \mathrm{F}_{1}^{2}\left(x, e_{i}\right) \otimes e_{i}\right\}
\end{gathered}
$$

Remark 14.9. The tensor $C$ will be called the double-stress tensor.

Remark 14.10. Imposing the left side symmetry of $C$ (as done in Seppecher (1987)) may seem complicated and artificial but the following Theorem 14.16 will show the advantage of such a choice.

The representation formula for the tensor $C$ obtained in equation 211 allows us to prove also the following

Corollary 14.11. For a generic dihedral shape $\left(n_{1}, n_{2}, \tau\right)$ the edge contact forces can be represented by

$$
\mathrm{F}_{0}^{1}\left(x,\left(n_{1}, n_{2}, \tau\right)\right)=\left(C(x) \cdot n_{1}\right) \cdot \nu_{1}+\left(C(x) \cdot n_{2}\right) \cdot \nu_{2},
$$

where

$$
\nu_{1}=\tau \times n_{1} \quad \nu_{2}=-\tau \times n_{2} .
$$


Proof. Let us first consider the dihedral shapes $f_{1}=\left(-e_{2},-e_{3}, e_{1}\right), f_{2}=$ $\left(-e_{3},-e_{1}, e_{2}\right)$ and $f_{3}=\left(-e_{1},-e_{2}, e_{3}\right)$ where $\left\{e_{i}\right\}$ is an orthonormal basis. With simple calculations we get from 211

$$
\left(C(x) .-e_{2}\right) .-e_{3}=\frac{1}{2} \mathrm{~F}_{0}^{1}\left(x, f_{1}\right) \quad\left(C(x) .-e_{3}\right) .-e_{2}=\frac{1}{2} \mathrm{~F}_{0}^{1}\left(x, f_{1}\right)
$$

which implies 213 for dihedral shapes verifying the conditions

$$
n_{1} \cdot n_{2}=0 ; \quad \tau \cdot n_{1}=0 ; \quad \tau \cdot n_{2}=0 .
$$

For getting the statement for a generic dihedral shape it is enough to consider the $\varepsilon$-families of 3 -prisms with height parallel to the vector $\tau$ the bases of which are triangles with two sides ortogonal to $n_{1}$ and $n_{2}$ and having a right angle.

A first important consequence of the representation formula 211 can be obtained by means of a very simple reasoning. Indeed we can get the following lemma, which has been formulated already in dell'Isola and Seppecher (1995)-(1997):

Lemma 14.12. The form of surface 2-forces compatible with vanishing line 1-forces. When $\mathrm{F}_{0}^{1}$ is vanishing equations (210) and (211) imply that

$$
\begin{aligned}
& \left(C\left(x_{0}\right) \cdot n\right) \cdot n=\mathrm{F}_{1}^{2}\left(x_{0}, n\right)= \\
= & \sum_{i=1}^{3} \mathrm{~F}_{1}^{2}\left(x_{0}, e_{i}\right)\left(n \cdot e_{i}\right)^{2}= \\
= & \sum_{j=1}^{3} \mathrm{~F}_{1}^{2}\left(x_{0}, e_{j}^{\prime}\right)\left(n \cdot e_{j}^{\prime}\right)^{2}
\end{aligned}
$$

where $\left\{e_{i}\right\}$ and $\left\{e_{j}^{\prime}\right\}$ are two generic orthonormal bases. As the found expression is valid for every orthonormal vector basis, we can conclude that if $\mathrm{F}_{0}^{1}$ is vanishing then $\mathrm{F}_{1}^{2}$ does not depend on $n$ : this is the only case in which the 1-normal surface distributions (i.e. surface 2-forces) can be nonzero with vanishing edge forces.

\subsection{Representation theorems for contact 1 -forces. Cauchy stress tensor.}

To proceed we need to apply Gauss divergence Theorem to some tensor fields. Therefore we are guided to add some further regularity assumptions on the considered stress state $\mathfrak{S}$, which for second gradient continua has the form $\left\{F_{0}^{2}, F_{1}^{2}, F_{0}^{1}\right\}$. 
Although a careful analysis (which will be included in a forthcoming paper) is possible, leading us to the determination of the most adapted functional space to be chosen to "host" considered fields, for seek of simplicity and in order not to distract the reader with presently unessential mathematical formalism, we limit ourselves to the consideration of very regular stress states.

\section{Hypothesis of $C^{1}$ regularity.}

For every given regular shape $f$ and every dihedral shape $d$, the partial functions

$$
\mathrm{F}_{1}^{2}(\cdot, f), \quad \mathrm{F}_{0}^{1}(\cdot, d)
$$

are $C^{1}$ functions.

As an obvious consequence of this assumption one easily gets that the tensor field $C$ defined in 14.8 is also a field of class $C^{1}$.

Remark 14.13. We now can show how our hypothesis (174), which implies but is not at all equivalent to hypothesis (138) put forward by Noll and Virga (1990), allows us to prove the assumption III on page 21 in just mentioned paper, and to show that the example treated in its Section 9 actually is exhaustive of all possible cases.

Indeed the following two Theorems give the general representation of edge and surface contact forces. We prove them starting from the quasi balance of power and under the regularity assumptions 10.1 to which we add the last assumed hypothesis 14.3.

Theorem 14.14. Let $S$ be the contact surface of an admissible domain $V$. Let $x$ be a regular point of an edge of $S$. Let $\left(n_{1}, n_{2}, \tau\right)$ be the tangent dihedral shape to $S$ at $x$. Then the edge force density at $x$ depends only on $\left(n_{1}, n_{2}, \tau\right)$ and is represented in terms of the second order stress tensor by

$$
\mathrm{F}_{0}^{1}(x,[(x, S)])=\left(C(x) \cdot n_{1}\right) \cdot \nu_{1}+\left(C(x) \cdot n_{2}\right) \cdot \nu_{2},
$$

where we have introduced the following notations:

$$
\nu_{1}=\tau \times n_{1} \quad \nu_{2}=-\tau \times n_{2} .
$$

Remark 14.15. The observed arbitrariness regarding the tensor $C$ has no influence on the representation formula (224) as the "shape" tensor

$$
n_{1} \otimes \nu_{1}+n_{2} \otimes \nu_{2}
$$

at any edge actually is a symmetric tensor. 
Theorem 14.16. At any regular point of the surface $S$ it exists a continuous second order tensor field $T$ such that

$$
\mathrm{F}_{0}^{2}(x,[(x, S)])=T(x) \cdot n-\operatorname{div}_{s}((C(x) \cdot n) . \Pi),
$$

where $\Pi$ denotes the projector on the tangent plane to the surface $S$

$$
(\Pi=I d-n \otimes n) .
$$

Remark 14.17. The arbitrariness in $C$ has an influence on $T$. With the choice (221), the tensor $T$ is symmetric.

Proof. We prove at the same time both Theorems 14.14 and 14.16. Because of Theorem 14.8, inequality (174) may be written

$$
\begin{gathered}
\mid \int_{L^{r}} \mathrm{~F}_{0}^{1}(x,[(x, S)]) \cdot U(x) d l+\int_{S^{r}} \mathrm{~F}_{0}^{2}(x,[(x, S)]) \cdot U(x) d s+ \\
+\int_{S^{r}}((C(x) \cdot n) \cdot n) \cdot \frac{\partial U}{\partial n}(x) d s\left|<K_{U}\right| V \mid .
\end{gathered}
$$

Due to the equality

$$
\left|\int_{V} \operatorname{div}(\nabla U . . C) d v\right|=\left|\int_{S^{r}} \nabla U . .(C . n) d s\right|,
$$

the quantity

$$
\int_{S^{r}} \nabla U . .(C . n) d s
$$

is quasi-balanced. Then there exists a scalar $K_{U}^{\prime}$ such

$$
\begin{aligned}
& \mid \int_{L^{r}} \mathrm{~F}_{0}^{1}(x,[(x, S)]) \cdot U(x) d l+\int_{S^{r}} \mathrm{~F}_{0}^{2}(x,[(x, S)]) \cdot U(x) d s+ \\
+ & \int_{S^{r}}\left\{((C(x) \cdot n) \cdot n) \cdot \frac{\partial U}{\partial n}(x)-\nabla U(x) . .(C . n)\right\} d s\left|<K_{U}^{\prime}\right| V \mid .
\end{aligned}
$$

Using now the decomposition

$$
\nabla U . .(C . n)=\partial U / \partial n .((C . n) . n)+\nabla^{s} U . .((C . n) . \Pi)
$$

where $\nabla^{s}$ denotes the surface gradient on $S$, we get

$$
\begin{gathered}
\mid \int_{L^{r}} \mathrm{~F}_{0}^{1}(x,[(x, S)]) \cdot U(x) d l+\int_{S^{r}} \mathrm{~F}_{0}^{2}(x,[(x, S)]) \cdot U(x) d s+ \\
\quad+\int_{S^{r}} \nabla^{s} U(x) . .((C(x) \cdot n) \cdot \Pi) d s\left|<K_{U}^{\prime}\right| V \mid .
\end{gathered}
$$


We apply the divergence theorem on every face of $S$, so obtaining

$$
\left|\int_{L^{r}} \mathcal{F}^{\prime}(x,[(x, S)]) \cdot U(x) d l+\int_{S^{r}} F^{\prime}(x,[(x, S)]) \cdot U(x) d s\right|<K_{U}^{\prime}|V|,
$$

where we used the following definitions $\mathrm{F}_{0}^{2}, \mathrm{~F}_{1}^{2}, \mathrm{~F}_{0}^{1}$

$$
\begin{aligned}
& \mathcal{F}^{\prime}(x,[(x, S)]):=\mathrm{F}_{0}^{1}(x,[(x, S)])-\left(C(x) \cdot n_{1}\right) \cdot \nu_{1}+\left(C(x) \cdot n_{2}\right) \cdot \nu_{2}, \\
& F^{\prime}(x,[(x, S)]):=\mathrm{F}_{0}^{2}(x,[(x, S)])+\nabla^{s} \cdot((C(x) \cdot n) \cdot \Pi) .
\end{aligned}
$$

Because of the regularity hypotheses added in this section, $F^{\prime}$ and $\mathcal{F}^{\prime}$ represent a stress state verifying the assumptions listed in 11. Because of inequality 231, we may apply to them our results found in 11 . Theorem 11.7 implies that $\mathcal{F}^{\prime}(x,[(x, S)])$ is vanishing and Theorem ?? establishes the existence of a continuous second order tensor field $T(\cdot)$ such that

$$
F^{\prime}(x,[(x, S)])=T(x) \cdot n .
$$

\subsection{Surface N-th order Forces}

In this section we indicate how the results obtained in the previous subsection devoted to surface double forces can be further generalized.

\section{Dependence of $n$-th order stress functions on the shape of the contact surface. A theorem analogous to Noll Theorem.}

We start with action-reaction theorem for surface $N$-forces and edge $N-1$ forces in $N-t h$ gradient continua.

Theorem 14.18. Let us consider a stress state $\mathfrak{S}$ of grade $N$. At every point $x$, for all plane shape $n$, for all dihedral shape $\left(n, e_{1}, e_{2}\right)$ and for all polihedral shape $\left(n_{1}, n_{2}, \ldots ., n_{k}\right)$ we have

$$
\begin{gathered}
\mathrm{F}_{N-1}^{2}(x, n)+(-1)^{N-1} \mathrm{~F}_{N-1}^{2}\left(x_{0},-n\right)=0 \\
\mathrm{~F}_{N-2}^{1}\left(x,\left(n,-e_{1}, e_{2}\right)\right)+(-1)^{N-2} \mathrm{~F}_{N-2}^{1}\left(x,\left(n, e_{1}, e_{2}\right)\right)=0 ; \\
\mathrm{F}_{N-2}^{1}\left(x,\left(-n, e_{1}, e_{2}\right)\right)+(-1)^{N-2} \mathrm{~F}_{N-2}^{1}\left(x,\left(n, e_{1}, e_{2}\right)\right)=0 ; \\
\mathrm{F}_{N-2}^{1}\left(x,\left(n, e_{1},-e_{2}\right)\right)+(-1)^{N-2} \mathrm{~F}_{N-2}^{1}\left(x,\left(n, e_{1}, e_{2}\right)\right)=0 \\
\mathrm{~F}_{N-3}^{0}\left(x,\left(-n_{1}, \widehat{-n_{2}, \ldots .},-n_{k}\right)\right)+\mathrm{F}_{N-3}^{0}\left(x,\left(n_{1}, \widehat{n_{2}, \ldots}, n_{k}\right)=0\right.
\end{gathered}
$$


Proof. The reasoning proceeds exactly as in the case of stress states of grade 2,with a difference: while we still consider the coordinate system $\left(x_{0}, e_{1}, e_{2}, n\right)$ and the domain

$$
C_{\varepsilon}=[0, \varepsilon] \times[0, \varepsilon] \times\left[0, \varepsilon^{2}\right]
$$

we use instead the vector field (where $U_{0}$ is a given vector)

$$
U: x \mapsto\left(x_{3}\right)^{N-1} U_{0}
$$

which has as $(N-1)-t h$ gradient the function

$$
\nabla^{N-1} U: x \mapsto(N-1) ! U_{0} \otimes \underbrace{n \ldots \otimes n}_{(N-1)-\text { times }}=:(N-1) ! U_{0} \otimes n^{N-1}
$$

The shapes of $\partial C_{\varepsilon}$ are prescribed shapes therefore the functions $\mathrm{F}$ in the stress state $\mathfrak{S}$ are uniformly bounded in the set

$$
\bigcup_{\varepsilon>0}\left(C_{\varepsilon} \times \Phi\left(\partial C_{\varepsilon}\right)\right) \text {. }
$$

Considering the area or length of each face or edge, as in the previous theorem 14.6 the inequality 169 applied to $C_{\varepsilon}$ implies together with the expression 168 (all other terms in 169 in the limit are vanishing faster than $\left.\varepsilon^{2}\right)$

$$
\begin{gathered}
\lim _{\varepsilon \longrightarrow 0} \varepsilon^{-2} \int_{S_{\varepsilon}^{+}} F_{N-1}^{2}(x, n) \cdot U_{0} d s+ \\
+\lim _{\varepsilon \longrightarrow 0} \varepsilon^{-2} \int_{S_{\varepsilon}^{-}}(-1)^{N-1} F_{N-1}^{2}(x,-n) \cdot U_{0} d s=0,
\end{gathered}
$$

where $S_{\varepsilon}^{+}$and $S_{\varepsilon}^{-}$denote the upper and lower faces of $C_{\varepsilon}$ i.e. in formulas

$$
S^{+}=\left\{x: x \cdot e_{3}=+\varepsilon^{2}\right\}, S^{-}=\left\{x: x \cdot e_{3}=0\right\}
$$

The regularity assumptions accepted for $\mathrm{F}_{N-1}^{2}$ and the arbitrariness of $U_{0}$ finally imply

$$
\mathrm{F}_{N-1}^{2}(x, n)+(-1)^{N-1} \mathrm{~F}_{N-1}^{2}\left(x_{0},-n\right)=0 .
$$

Repeating then the construction conceived for dihedral shapes in Theorem 14.6 we easily get also 234 . Finally in order to get 235 one needs to consider the $\varepsilon^{2}$-translation of the considered polihedral shape and construct the $\varepsilon$-family of volumes so obtained. 
We are now able to prove that in $N-t h$ gradient continua contact surface $N$ - forces and edge $(N-1)$-forces on any couple of contact surface which are tangent assume the same value.

Theorem 14.19. Let us consider a $N$-th gradient continuum. Under regularity assumptions 10.1 and having postulated the quasi-balance of contact actions, we have that: i) at every point $x$ and for every regular shape $f$ tangent to the plane shape $n$ we have

$$
\mathrm{F}_{N-1}^{2}(x, f)=\mathrm{F}_{N-1}^{2}(x, n)
$$

that is $\mathrm{F}_{1}^{2}$ at surface regular points depends on the shape of the contact surface only through its normal ii) at every point $x$ and for every regular edge shape $f$ tangent to the dihedral shape $\left(n_{1}, n_{2}, e_{1}\right)$ we have

$$
\mathrm{F}_{N-2}^{1}(x, f)=\mathrm{F}_{N-2}^{1}\left(x,\left(n_{1}, n_{2}, e_{1}\right)\right) .
$$

that is $\mathrm{F}_{N-2}^{1}$ at edge points depends on the shape of the contact surface only through the tangent dihedral shape; iii) at every point $x$ and for every wedge shape $f$ tangent to the nondegenerate Polihedral Wedge Shape $\left(n_{1}, \ldots, n_{k}\right)$ we have

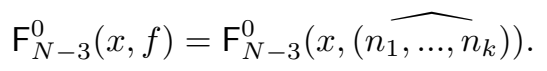

that is $\mathrm{F}_{N-3}^{0}$ at wedge points depends on the shape of the contact surface only through the tangent polihedral shape.

Proof. The proof of 241 is very close to that we have given for the Theorem 12.5. The $\varepsilon$-family to be considered is exactly the same: the only difference to be adopted in the demonstration consists in the need of using as test function in the quasi-balance of power the vector field

$$
U: x \longrightarrow\left(x \cdot n_{0}\right)^{N-1} U_{0}
$$

where $n_{0}$ denotes the normal to $S$ at $x_{0}$ and $U_{0}$ is a generic vector. On the other hand slightly different constructions are needed to prove 242 and 243: these constructions require the $\varepsilon$-translation of a neighborhood of the contact surface keeping fixed the tangent dihedral or polihedral shape.

\subsection{A Representation Theorem for surface $(N-1)$-forces gen- eralizing the Cauchy tetrahedron Theorem.}

Celebrated Cauchy tetrahedron argument deserves to be admired. It allows us to prove also for $N-t h$ gradient continua a representation theorem for contact $(N-1)$-forces in terms of a tensor of order $N+1$. Indeed 
$N$-force surface density (which has been seen to depend only on the tangent planar shape to the contact surface) can be obtained by calculating a vector-valued polynomial function having grade exactly $N$.

Theorem 14.20. Let $B$ a body occupying in one of its configurations the domain D. Let us consider a physically admissible stress state $\mathfrak{S}$ of grade $N$. Let us assume the regularity assumptions 10.1. If quasi balance of power holds then there exists a continuous tensor field $C_{N}$ of order $N+1$ such that, at any point $x_{h} \in D$ and for any plane shape $n$,

$$
\mathrm{F}_{N-1}^{2}\left(x_{h}, n\right)=(C_{N}\left(x_{h}\right) \underbrace{. n) \ldots . . . n}_{N}
$$

Proof. We adapt the Cauchy tetrahedron construction. We consider the point $x_{0}$ and the three unit vectors $\left(e_{1}, e_{2}, e_{3}\right)$. We define the tetrahedron $V$ as follows i) its face $S$ opposed to the vertex in $x_{0}$ is normal to the unit vector $n$; ii) its edges

$$
L_{i}=\overline{x_{0} x_{i}}=l_{i} e_{i} \quad(i=1,2,3)
$$

are such that $x_{i} \in S$; iii) its height $H$ parallel to $n$ has length $h$ : if we call $x_{h}$ the second endpoint of the segment $H$ we have

$$
H=\overline{x_{0} x_{h}} ; \quad x_{h}-x_{0}=h n .
$$

It is easy to check that: i) the equation of the plane $\pi_{S}$ orthogonal to $n$ and passing through the point $x_{h}$ is given by

$$
\left(x-x_{h}\right) \cdot n=0
$$

ii) as $S \subset \pi_{S}$ and $x_{i} \in S$ then

$$
\left(x_{0}+l_{i} e_{i}-x_{h}\right) \cdot n=0 \Rightarrow-h+l_{i} e_{i} \cdot n=0 \Rightarrow h=l_{i} e_{i} \cdot n \Rightarrow \frac{h}{e_{i} \cdot n}=l_{i}
$$

iii) the edges of the tetrahedron $V$ lying in the plane $\pi_{S}$ are

$$
\begin{aligned}
& \overrightarrow{x_{1} x_{2}}=l_{2} e_{2}-l_{1} e_{1}=h\left(\frac{e_{2}}{e_{2} \cdot n}-\frac{e_{1}}{e_{1} \cdot n}\right) \\
& \overrightarrow{x_{2} x_{3}}=l_{3} e_{3}-l_{2} e_{2}=h\left(\frac{e_{3}}{e_{3} \cdot n}-\frac{e_{2}}{e_{2} \cdot n}\right) \\
& \overrightarrow{x_{3} x_{1}}=l_{1} e_{1}-l_{3} e_{3}=h\left(\frac{e_{1}}{e_{1} \cdot n}-\frac{e_{3}}{e_{3} \cdot n}\right)
\end{aligned}
$$

iv) the triangles $S_{3}, S_{1}$ and $S_{2}$ having a vertex in $x_{0}$ and respectively $\overline{x_{1} x_{2}}$ $, \overline{x_{2} x_{3}}, \overline{x_{3} x_{1}}$ as side opposed to $x_{0}$ have as outward pointing unit normals 
respectively the vectors

$$
n_{3}=\frac{e_{2} \times e_{1}}{\left|e_{2} \times e_{1}\right|}, \quad n_{1}=\frac{e_{3} \times e_{2}}{\left|e_{3} \times e_{2}\right|}, \quad n_{2}=\frac{e_{1} \times e_{3}}{\left|e_{1} \times e_{3}\right|} ;
$$

It is important to establish the relationship between the reciprocal basis $\left(e^{j}\right)$ of the introduced basis $\left(e_{i}\right)$ and the three normals $\left(n_{j}\right)$. Using the notation $v:=e_{1} \cdot\left(e_{2} \times e_{3}\right)$ we have:

$$
v e^{1}=e_{2} \times e_{3} ; \quad v e^{2}=e_{3} \times e_{1} ; \quad v e^{3}=e_{1} \times e_{2} ; \quad-n_{j}=\frac{e^{j}}{\left|e^{j}\right|}
$$

v) the areas of the faces $S, S_{3}, S_{1}$ and $S_{2}$ can be easily calculated. We can verify some simple relationships:

$$
\begin{aligned}
& 2|S|=\overrightarrow{x_{1} x_{2}} \times \overrightarrow{x_{1} x_{3}} \cdot n=h^{2}\left(\frac{e_{2}}{e_{2} \cdot n}-\frac{e_{1}}{e_{1} \cdot n}\right) \times\left(-\frac{e_{1}}{e_{1} \cdot n}+\frac{e_{3}}{e_{3} \cdot n}\right) \cdot n= \\
& =h^{2}\left(\frac{e_{2}}{e_{2} \cdot n} \times\left(-\frac{e_{1}}{e_{1} \cdot n}+\frac{e_{3}}{e_{3} \cdot n}\right)-\frac{e_{1}}{e_{1} \cdot n} \times\left(\frac{e_{3}}{e_{3} \cdot n}\right)\right) \cdot n= \\
& =h^{2}\left(\frac{-\left(e_{2} \times e_{1}\right) \cdot n}{\left(e_{2} \cdot n\right)\left(e_{1} \cdot n\right)}+\frac{\left(e_{2} \times e_{3}\right) \cdot n}{\left(e_{2} \cdot n\right)\left(e_{3} \cdot n\right)}+\frac{-\left(e_{1} \times e_{3}\right) \cdot n}{\left(e_{1} \cdot n\right)\left(e_{3} \cdot n\right)}\right)= \\
& =h^{2} v\left(\frac{e^{3} \cdot n}{\left(e_{2} \cdot n\right)\left(e_{1} \cdot n\right)}+\frac{e^{1} \cdot n}{\left(e_{2} \cdot n\right)\left(e_{3} \cdot n\right)}+\frac{e^{2} \cdot n}{\left(e_{1} \cdot n\right)\left(e_{3} \cdot n\right)}\right)= \\
& =\frac{h^{2} v}{\left(e_{3} \cdot n\right)\left(e_{2} \cdot n\right)\left(e_{1} \cdot n\right)}\left(\sum_{i=1}^{3}\left(e^{i} \cdot n\right)\left(e_{i} \cdot n\right)\right) \\
& =\frac{h^{2}\left(e_{1} \cdot\left(e_{2} \times e_{3}\right)\right)}{\left(e_{3} \cdot n\right)\left(e_{2} \cdot n\right)\left(e_{1} \cdot n\right)} \\
& 2|S|=\frac{v h^{2}}{\left(e_{2} \cdot n\right)\left(e_{1} \cdot n\right)\left(e_{3} \cdot n\right)} \\
& 2\left|S_{3}\right|=\left(l_{2} e_{2} \times l_{1} e_{1}\right) \cdot n_{3}=h^{2}\left(\frac{e_{2}}{e_{2} \cdot n} \times \frac{e_{1}}{e_{1} \cdot n}\right) \cdot n_{3} \\
& =h^{2} \frac{\left|e_{2} \times e_{1}\right|}{\left(e_{2} \cdot n\right)\left(e_{1} \cdot n\right)}=\frac{h^{2} v\left|e^{3}\right|}{\left(e_{2} \cdot n\right)\left(e_{1} \cdot n\right)} \\
& 2\left|S_{1}\right|=\frac{v h^{2}\left|e^{1}\right|}{\left(e_{2} \cdot n\right)\left(e_{3} \cdot n\right)} ; \quad 2\left|S_{2}\right|=\frac{v h^{2}\left|e^{2}\right|}{\left(e_{1} \cdot n\right)\left(e_{3} \cdot n\right)}
\end{aligned}
$$

so that we have

$$
|S|=\frac{\left|S_{i}\right|}{\left(e_{i} \cdot n\right)\left|e^{i}\right|} ; \quad(i=1,2,3)
$$


vi) the shapes of the edges $L_{1}, L_{2}$ and $L_{3}$ are respectively $f_{1}:=\left(n_{2}, n_{3}, e_{1}\right)$ , $f_{2}:=\left(n_{3}, n_{1}, e_{2}\right)$ and $f_{3}:=\left(n_{1}, n_{2}, e_{3}\right)$. Let $V^{\varepsilon}$ be the image of $V$ under an homothetic transformation of ratio $\varepsilon$ and center $x_{h}$, we denote by $L^{\varepsilon}$ and $S^{\varepsilon}$ (with eventually suitable subscripts) the images of all edges and surfaces previously introduced. We will use now the quasi-balance of contact power for the fixed (it does not vary with $\varepsilon$ !) test field

$$
U: x \longrightarrow\left(\left(x-x_{h}\right) \cdot n\right)^{N-1} U_{0}
$$

where $U_{0}$ is a generic vector and for the varying family of domains $V^{\varepsilon}$, its contact surface, having the edges and wedges we have just described. The equation 168 applied to state the quasi-balance of power for a physically admissible stress state $\mathfrak{S}$ in the domain $V_{\varepsilon}$ becomes

$$
\begin{array}{r}
\left|\mathcal{P}_{U}\left(\mathfrak{S}, V_{\varepsilon}\right)\right|:=\mid \int_{\mathcal{S}_{r}^{\varepsilon}} \mathrm{F}_{0}^{2} \cdot U+\int_{\mathcal{L}_{r}^{\varepsilon}} \mathrm{F}_{0}^{1} \cdot U+\sum_{k=1}^{N-1} \int_{\mathcal{S}_{r}^{\varepsilon}} \mathrm{F}_{k}^{2} \cdot\left(\nabla^{k} U\right)_{\perp}+ \\
+\sum_{k=1}^{N-2} \int_{\mathcal{L}_{r}^{\varepsilon}} \mathrm{F}_{k}^{1} \cdot\left(\nabla^{k} U\right)_{\perp}+\sum_{k=0}^{N-3} \int_{\mathcal{W}_{S}^{\varepsilon}} \mathrm{F}_{k}^{0} \cdot \nabla^{k} U \mid \leq K_{U} \varepsilon^{3}
\end{array}
$$

where with the symbols $\mathcal{S}_{r}^{\varepsilon}, \mathcal{L}_{r}^{\varepsilon}$ and $\mathcal{W}_{S}^{\varepsilon}$ we have denoted respectively the set of regular surface points, regular edges points and wedges belonging to the contact surface of the domain $V_{\varepsilon}$. We now start by remarking that the field $U$ vanishes on the surface $S^{\varepsilon}$ together with all its derivatives up to the order $N-2$.Indeed:

$$
\begin{gathered}
\nabla^{k} U=\frac{(N-1) !}{(N-1-k) !}\left(\left(x-x_{h}\right) \cdot n\right)^{N-1-k} U_{0} \otimes n^{k} \\
\left(\nabla^{k} U\right)_{\perp}=\frac{(N-1) !}{(N-1-k) !}\left(\left(x-x_{h}\right) \cdot n\right)^{N-1-k} U_{0} \otimes\left(n-\left(n \cdot e_{i}\right) e_{i}\right)^{k}
\end{gathered}
$$

We now consider one by one all the addends in 275 in which the previous 
equalities have been replaced

$$
\begin{aligned}
& \left|\int_{\mathcal{S}_{r}^{\varepsilon}} \mathrm{F}_{0}^{2} \cdot U\right|= \\
& =\left|\sum_{i=1}^{3} \int_{\mathcal{S}_{i}^{\varepsilon}} \mathrm{F}_{0}^{2} \cdot\left(\left(x-x_{h}\right) \cdot n\right)^{N-1} U_{0}\right| \leq K_{0}^{2}\left\|U_{0}\right\| \varepsilon^{2} \varepsilon^{N-1} \\
& (\forall k<N-1)\left(\begin{array}{c}
\left|\int_{\mathcal{S}_{r}^{\varepsilon}} \mathrm{F}_{k}^{2} \cdot\left(\nabla^{k} U\right)_{\perp}\right|= \\
=\left|\sum_{i=1}^{3} \int_{S_{i}^{\varepsilon}} \mathrm{F}_{k}^{2} \cdot \frac{(N-1) !}{(N-1-k) !}\left(\left(x-x_{h}\right) \cdot n\right)^{N-1-k} U_{0}\left(n \cdot n_{i}\right)^{k}\right| \\
\leq K_{k}^{2}\left\|U_{0}\right\| \varepsilon^{2} \varepsilon^{N-1-k}
\end{array}\right) \\
& \left|\int_{\mathcal{S}_{r}^{\varepsilon}} \mathrm{F}_{N-1}^{2} \cdot\left(\nabla^{N-1} U\right)_{\perp}\right|=\mid \sum_{i=1}^{3} \int_{S_{i}^{\varepsilon}} \mathrm{F}_{N-1}^{2} \cdot(N-1) ! U_{0}\left(n \cdot n_{i}\right)^{N-1} \\
& +\int_{S^{\varepsilon}} \mathrm{F}_{N-1}^{2} \cdot(N-1) ! U_{0} \leq K_{N-1}^{2}\left\|U_{0}\right\| \varepsilon^{2} \\
& \left|\int_{\mathcal{L}_{r}^{\varepsilon}} \mathrm{F}_{0}^{1} \cdot U\right|=\left|\sum_{i=1}^{3} \int_{L_{i}^{\varepsilon}} \mathrm{F}_{0}^{1} \cdot\left(\left(x-x_{h}\right) \cdot n\right)^{N-1} U_{0}\right| \leq K_{0}^{1}\left\|U_{0}\right\| \varepsilon^{1} \varepsilon^{N-1} \\
& (\forall k<N-2)\left(\left|\int_{\mathcal{L}_{r}^{\varepsilon}} \mathrm{F}_{k}^{1} \cdot\left(\nabla^{k} U\right)_{\perp}\right|=\right. \\
& \begin{array}{c}
=\mid \sum_{i=1}^{3} \int_{L_{i}^{\varepsilon}} \mathrm{F}_{k}^{1} \cdot\left(\frac{(N-1) !}{(N-1-k) !}\right. \\
\left.\left.\left(\left(x-x_{h}\right) \cdot n\right)^{N-1-k} U_{0} \otimes\left(n-\left(n \cdot e_{i}\right) e_{i}\right)^{k}\right) \mid \leq\right) \\
\leq K_{k}^{1}\left\|U_{0}\right\| \varepsilon^{1} \varepsilon^{N-1-k}
\end{array} \\
& \left|\int_{\mathcal{L}_{r}^{\varepsilon}} \mathrm{F}_{N-2}^{1} \cdot\left(\nabla^{N-2} U\right)_{\perp}\right|= \\
& =\left|\sum_{i=1}^{3} \int_{L_{i}^{\varepsilon}} \mathrm{F}_{N-2}^{1} \cdot\left(\left((N-1) !\left(\left(x-x_{h}\right) \cdot n\right) U_{0} \otimes\left(n-\left(n \cdot e_{i}\right) e_{i}\right)^{N-2}\right)\right)\right| \\
& \leq K_{N-2}^{1}\left\|U_{0}\right\| \varepsilon^{2} \\
& (\forall k \leq N-3)\left(\begin{array}{c}
\left|\int_{\mathcal{W}_{S}^{\varepsilon}} \mathrm{F}_{k}^{0} \cdot \nabla^{k} U\right|= \\
\left|\mathrm{F}_{k}^{0}\left(x_{0},\left[\left(x_{0}, S^{\varepsilon}\right)\right]\right) \cdot\left(\frac{(N-1) !\left(\left(x-x_{h}\right) \cdot n\right)^{N-1-k}}{N-1-k !}\right) U_{0} \otimes n^{k}\right| \leq \\
\leq K_{0}^{0}\left\|U_{0}\right\| \varepsilon^{N-1-k}
\end{array}\right)
\end{aligned}
$$


We now simply multiply both terms in 261 (in which we have replaced 259) times $\varepsilon^{-2}$. Then we take into account all inequality listed from 262 to 272 and we remark that the shapes on every face and edge of the boundary $\partial V^{\varepsilon}$ are spatially constants and, together with wedge shapes, also independent of $\varepsilon$. We are thus able to calculate the limit for $\varepsilon$ tending to zero and finally get (remark that having already treated the case $N=2$, we can consider $N \geq 3)$

$$
\begin{gathered}
\lim _{\varepsilon \rightarrow 0} \varepsilon^{-2}\left(\sum_{i=1}^{3} \int_{S_{i}^{\varepsilon}} \mathrm{F}_{N-1}^{2} \cdot(N-1) ! U_{0}\left(n \cdot n_{i}\right)^{N-1}+\right. \\
+\int_{S^{\varepsilon}} \mathrm{F}_{N-1}^{2} \cdot(N-1) ! U_{0}+ \\
+\sum_{i=1}^{3} \int_{L_{i}^{\varepsilon}} \mathrm{F}_{N-2}^{1} \cdot\left(\left((N-1) !\left(\left(x-x_{h}\right) \cdot n\right) U_{0} \otimes\left(n-\left(n \cdot e_{i}\right) e_{i}\right)^{N-2}\right)\right)+ \\
\left.+\mathrm{F}_{N-3}^{0}\left(x_{h}-\varepsilon h n,\left[\left(x_{h}-\varepsilon h n, S^{\varepsilon}\right)\right]\right) \cdot\left(\frac{(N-1) !(\varepsilon h)^{2}}{2 !}\right) U_{0} \otimes n^{N-3}\right)=0
\end{gathered}
$$

Before starting to calculate the indicated limit we remark that the set of shapes $\bigcup \Phi\left(\partial V^{\varepsilon}\right)$ is a prescribed set of shapes, so that all the continuous functions $F$ are equi-uniformly bounded. The previous equality, where we have now explicitly indicated the argument of the functions $F$ and once we apply the first mean value theorem for integration, becomes

$$
\begin{aligned}
\lim _{\varepsilon \rightarrow 0} \varepsilon^{-2} & \left(\sum_{i=1}^{3}\left|S_{i}^{\varepsilon}\right| \mathrm{F}_{N-1}^{2}\left(x_{i}^{\varepsilon}, n_{i}\right) \cdot(N-1) ! U_{0}\left(n \cdot n_{i}\right)^{N-1}\right. \\
& +\left|S^{\varepsilon}\right| \mathrm{F}_{N-1}^{2}\left(x_{\varepsilon}, n\right) \cdot(N-1) ! U_{0}+ \\
& +\sum_{i=1}^{3}\left(\int_{L_{i}^{\varepsilon}}\left(\left(x-x_{h}\right) \cdot n\right)\right) \mathrm{F}_{N-2}^{1}\left(\tilde{x}_{i}^{\varepsilon}, f_{i}\right) \\
& \cdot\left(\left((N-1) ! U_{0} \otimes\left(n-\left(n \cdot e_{i}\right) e_{i}\right)^{N-2}\right)\right)+ \\
+\mathrm{F}_{N-3}^{0}\left(x_{h}-\right. & \left.\left.\varepsilon h n,\left(\widehat{n_{1}, n_{2}, n_{3}}\right)\right) \cdot\left(\frac{(N-1) !(\varepsilon h)^{2}}{2 !}\right) U_{0} \otimes n^{N-3}\right)=0
\end{aligned}
$$

where $x_{i}^{\varepsilon} \in S_{i}^{\varepsilon}, \quad x_{\varepsilon} \in S^{\varepsilon}, \quad \tilde{x}_{i}^{\varepsilon} \in L_{i}^{\varepsilon}$. We now calculate the integral

$$
\int_{L_{i}^{\varepsilon}}\left(\left(x-x_{h}\right) \cdot n\right)=\frac{1}{2}\left(-e_{i} \cdot n\right) \varepsilon^{2} l_{i}^{2}
$$


and, as the following limit is finite

$$
\lim _{\varepsilon \rightarrow 0} \varepsilon^{-2}\left|S^{\varepsilon}\right|=\frac{v h^{2}}{2\left(e_{2} \cdot n\right)\left(e_{1} \cdot n\right)\left(e_{3} \cdot n\right)},
$$

we factorize $\left|S^{\varepsilon}\right|$ in equality 277 , to get

$$
\begin{gathered}
\lim _{\varepsilon \rightarrow 0}\left(\sum_{i=1}^{3}\left|S_{i}^{\varepsilon}\right|\left|S^{\varepsilon}\right|^{-1} \mathrm{~F}_{N-1}^{2}\left(x_{i}^{\varepsilon}, n_{i}\right) \cdot(N-1) ! U_{0}\left(n \cdot n_{i}\right)^{N-1}+\right. \\
+\mathrm{F}_{N-1}^{2}\left(x_{\varepsilon}, n\right) \cdot(N-1) ! U_{0}+ \\
+\sum_{i=1}^{3}\left(\frac{1}{2}\left(-e_{i} \cdot n\right) \varepsilon^{2} l_{i}^{2}\right)\left|S^{\varepsilon}\right|^{-1} \mathrm{~F}_{N-2}^{1}\left(\tilde{x}_{i}^{\varepsilon}, f_{i}\right) \cdot \\
\cdot\left((N-1) ! U_{0} \otimes\left(n-\left(n \cdot e_{i}\right) e_{i}\right)^{N-2}\right)+ \\
\left.\quad\left(\frac{(N-1) !(\varepsilon h)^{2}}{2 !}\right)\left|S^{\varepsilon}\right|^{-1}\left(\widehat{U_{0} \otimes n^{N-3}}\right)\right)=0
\end{gathered}
$$

To proceed we need i) to use the following formulas $(i=1,2,3)$

$$
\begin{gathered}
\left(e_{i} \cdot n\right)\left|e^{i}\right|=\left|S_{i}\right||S|^{-1}=\left|S_{i}^{\varepsilon}\right|\left|S^{\varepsilon}\right|^{-1} ; \quad \frac{h}{\left(e_{i} \cdot n\right)}=l_{i} \\
\varepsilon^{2} l_{i}^{2}\left|S^{\varepsilon}\right|^{-1}=l_{i}^{2}|S|^{-1}=v^{-1}\left(\frac{2\left(e_{2} \cdot n\right)\left(e_{1} \cdot n\right)\left(e_{3} \cdot n\right)}{\left(e_{i} \cdot n\right)^{2}}\right) \\
\left|S^{\varepsilon}\right|^{-1}(\varepsilon h)^{2}=h^{2}\left|S^{\varepsilon}\right|^{-1} \varepsilon^{2}=2 v^{-1}\left(e_{2} \cdot n\right)\left(e_{1} \cdot n\right)\left(e_{3} \cdot n\right)
\end{gathered}
$$

which are implied by 254 and 258 , ii) to simplify the nonvanishing factor $(N-1)$ ! and iii) to use simple algebra. We get:

$$
\begin{gathered}
\lim _{\varepsilon \rightarrow 0}\left(\sum_{i=1}^{3}\left(e_{i} \cdot n\right)\left|e^{i}\right| \mathrm{F}_{N-1}^{2}\left(x_{i}^{\varepsilon}, n_{i}\right) \cdot U_{0}\left(n \cdot n_{i}\right)^{N-1}+\right. \\
+\mathrm{F}_{N-1}^{2}\left(x_{\varepsilon}, n\right) \cdot U_{0} \\
+\sum_{i=1}^{3}\left(-v^{-1}\right) \frac{\left(e_{2} \cdot n\right)\left(e_{1} \cdot n\right)\left(e_{3} \cdot n\right)}{\left(e_{i} \cdot n\right)} \mathrm{F}_{N-2}^{1}\left(\tilde{x}_{i}^{\varepsilon}, f_{i}\right) \cdot \\
\cdot\left(U_{0} \otimes\left(n-\left(n \cdot e_{i}\right) e_{i}\right)^{N-2}\right)+ \\
\quad+\mathrm{F}_{N-3}^{0}\left(x_{h}-\varepsilon h n,\left(n_{1}, \widehat{n_{2},} n_{3}\right)\right) \cdot \\
\left.\cdot v^{-1}\left(e_{2} \cdot n\right)\left(e_{1} \cdot n\right)\left(e_{3} \cdot n\right)\left(U_{0} \otimes n^{N-3}\right)\right)=0
\end{gathered}
$$


It is now possible to calculate the limit and factorize the arbitrary vector $U_{0}$

$$
\begin{gathered}
\left(\sum_{i=1}^{3}\left(e_{i} \cdot n\right)\left|e^{i}\right| \mathrm{F}_{N-1}^{2}\left(x_{h}, n_{i}\right)\left(n \cdot n_{i}\right)^{N-1}+\mathrm{F}_{N-1}^{2}\left(x_{h}, n\right)+\right. \\
+\sum_{i=1}^{3}\left(-v^{-1}\right) \frac{\left(e_{2} \cdot n\right)\left(e_{1} \cdot n\right)\left(e_{3} \cdot n\right)}{\left(e_{i} \cdot n\right)} \mathrm{F}_{N-2}^{1}\left(x_{h}, f_{i}\right) \cdot\left(n-\left(n \cdot e_{i}\right) e_{i}\right)^{N-2}+ \\
\left.+v^{-1}\left(e_{2} \cdot n\right)\left(e_{1} \cdot n\right)\left(e_{3} \cdot n\right) \mathrm{F}_{N-3}^{0}\left(x_{h},\left(\widehat{n_{1}, n_{2}, n_{3}}\right)\right) \cdot n^{N-3}\right) \cdot U_{0}=0
\end{gathered}
$$

As the last equality holds for every nonvanishing vector $U_{0}$ it implies

$$
\begin{gathered}
\sum_{i=1}^{3}\left(e_{i} \cdot n\right)\left|e^{i}\right| \mathrm{F}_{N-1}^{2}\left(x_{h}, n_{i}\right)\left(n \cdot n_{i}\right)^{N-1}+\mathrm{F}_{N-1}^{2}\left(x_{h}, n\right)+ \\
+\sum_{i=1}^{3}\left(-v^{-1}\right) \frac{\left(e_{2} \cdot n\right)\left(e_{1} \cdot n\right)\left(e_{3} \cdot n\right)}{\left(e_{i} \cdot n\right)} \mathrm{F}_{N-2}^{1}\left(x_{h}, f_{i}\right) \cdot\left(n-\left(n \cdot e_{i}\right) e_{i}\right)^{N-2}+ \\
+v^{-1}\left(e_{2} \cdot n\right)\left(e_{1} \cdot n\right)\left(e_{3} \cdot n\right) \mathrm{F}_{N-3}^{0}\left(x_{h},\left(\widehat{n_{1}, n_{2}, n_{3}}\right)\right) \cdot n^{N-3}=0
\end{gathered}
$$

The formula which we have just obtained generalizes equation 209. As the following implication holds

$$
\left(\forall\left(e_{i}\right)\right)\left(\left(e_{i} \cdot e_{j}=\delta_{i j}\right) \Rightarrow(\forall i)\left(n_{i}=-e_{i}\right) \wedge(v=1)\right)
$$

then 292, together with theorem 14.18 obviously implies that there exists a tensor of order $N$ which allows for the representation of surface contact $(N-1)$-forces given in formula 245 .

Remark 14.21. When re-considering the flow of the demonstration of the previous theorem the reader will be persuaded that the tetrahedron argument can be used to get an alternative proof of Theorems 13.6 and 13.4.

The theorem which we have just proven leads us to define a tensor field $C_{N}$ of order $N+1$ such that

$$
\mathrm{F}_{N-1}^{2}\left(x_{h}, n\right)=(C_{N}\left(x_{h}\right) \underbrace{. n) \ldots .) . n}_{N \text { times }}=C_{N}\left(x_{0}\right) \cdot n^{N}
$$

This tensor is not uniquely determined, as only its right-side products by symmetric $(N+1)$ - th order tensors are determined. We may impose its right side symmetry and by using 292 we can easily prove the following 
Lemma 14.22. The only tensor field $C_{N}$ verifying the equation 294 and completely symmetric with respect its last $N$ indices has the following form

$$
\begin{gathered}
C_{N}(x)=\sum_{i=1}^{3}\left(\mathrm{~F}_{N-1}^{2}\left(x, n_{i}\right) \otimes\left(n_{i}\right)^{N-2}\right) \otimes \frac{1}{2}\left(e^{i} \otimes e_{i}+e_{i} \otimes e^{i}\right)+ \\
+\sum_{i=1}^{3} \mathrm{~F}_{N-2}^{1}\left(x, f_{i}\right)_{\perp} \otimes\left(v^{-1}\right) \frac{1}{2}\left(e_{i+1} \otimes e_{i+2}+e_{i+2} \otimes e_{i+1}\right)+ \\
-\mathrm{F}_{N-3}^{0}\left(x,\left(\widehat{n_{1}, n_{2}, n_{3}}\right)\right) \otimes v^{-1} \check{S}
\end{gathered}
$$

where we have introduced the third order tensor $S$ as follows $(\sigma(\{1,2,3\})$ denotes the set of all permutations in the set $\{1,2,3\}$ )

$$
\sum_{\pi \in \sigma(\{1,2,3\})} e_{\pi(1)} \otimes e_{\pi(2)} \otimes e_{\pi(3)}=: 6 \check{S}
$$

The previous Lemma allows us to state the following

Proposition 14.23. Given a physically admissible stress state $\mathfrak{S}$ of grade $N$. The tensor field $C_{N}$ the existence of which has been proven in the previous Theorem 14.20 allows for the representation of the highest order nonvanishing contact actions in $\mathfrak{S}$

$$
\mathrm{F}_{N-1}^{2}, \mathrm{~F}_{N-2}^{1}, \mathrm{~F}_{N-3}^{0}
$$

This representation is exactly the one given in equations 101,102 and 103.

\subsection{Representation theorem for contact $k$-forces with $k<N$.}

To proceed we will need to apply Gauss divergence Theorem to a sequence of tensor fields defined by recursively applying the previously presented tetrahedron argument. Indeed these tensor fields will be considered as arguments of the functionals introduced by formulas 105 .

Therefore we are naturally induced to add some further regularity assumptions on the considered physically admissible stress state $\mathfrak{S}$ of grade $N$. In the present work we limit ourselves to the consideration of very regular stress states.

\section{Hypothesis of $C^{K+1}$ regularity.}

For every given regular shape $f$ the partial functions

$$
\mathrm{F}_{K}^{2}(\cdot, f) \in \mathfrak{S}
$$


are $C^{K+1}$ functions.

As an obvious consequence of this assumption one easily gets that the tensor field $C_{N}$ defined in 14.20 is a field of class $C^{N}$.

We can now easily prove the following

Proposition 14.24. Let us consider a physically admissible stress state $\mathfrak{S}$ of grade $N$. The stress state $\mathfrak{S}:=\{\hat{\mathrm{F}}\}$ defined by

$$
\begin{gathered}
\hat{\mathrm{F}}_{J}^{2}:=\mathrm{F}_{J}^{2}-\mathfrak{F}\left(\partial B,\left\{0, \ldots 0, C_{N}\right\}, J\right) \\
\hat{\mathrm{F}}_{J}^{1}:=\mathrm{F}_{J}^{1}-\mathfrak{F}\left(\partial \partial B,\left\{0, \ldots 0, C_{N}\right\}, J\right) \\
\hat{\mathrm{F}}_{J}^{0}:=\mathrm{F}_{J}^{0}-\mathfrak{F}\left(\partial \partial \partial B,\left\{0, \ldots 0, C_{N}\right\}, J\right)
\end{gathered}
$$

is physically admissible, verifies the Hypothesis 14.6 and is a stress state of grade not greater than $N-1$.

Proof. The demonstration is obtained by remarking that because of 14.23 the definition 301 has all vanishing the actions $\left\{\hat{\mathrm{F}}_{N-1}^{2}, \hat{\mathrm{F}}_{N-2}^{1}, \hat{\mathrm{F}}_{N-3}^{0}\right\}$. Therefore the stress state $\mathfrak{S}$ cannot have a grade greater than $N-1$. Then the definition itself of the functional $\mathfrak{F}$ assures that $\mathfrak{S}$ verifies the regularity condition 14.6. Finally a simple integration by parts argument allows us to conclude that $\mathfrak{S}$ is physically admissible.

Definition 14.25. It is possible to apply recursively the tetrahedron argument and the previous proposition to defined the set of tensors $C_{K}$ where $1<k \leq N$.

Remark 14.26. Because of Hypothesis 14.6 the tensor fields $C_{K}$ have all the $k-t h$ order derivatives and these derivatives are continuous.

With a simple recursive argument it is then possible to prove the following

Theorem 14.27. Existence of Generalized Cauchy Stress Tensors. Given a physically admissible stress state $\mathfrak{S}=\left\{\mathrm{F}_{J}^{2}, \mathrm{~F}_{J}^{1}, \mathrm{~F}_{J}^{0}\right\}$ of grade $N$. Then the following representation formula holds

$$
\begin{gathered}
\mathrm{F}_{J}^{2}=\mathfrak{F}\left(\partial B,\left\{C_{2}, C_{3} \ldots, C_{N}\right\}, J\right) \\
\mathrm{F}_{J}^{1}=\mathfrak{F}\left(\partial \partial B,\left\{C_{2}, C_{3} \ldots, C_{N}\right\}, J\right) \\
\mathrm{F}_{J}^{0}=\mathfrak{F}\left(\partial \partial \partial B,\left\{C_{2}, C_{3} \ldots, C_{N}\right\}, J\right)
\end{gathered}
$$


In other words: for every physically admissible stress state of grade $N$ there exists a $N$-tuple of stress tensors which allow for the representation of all admissible contact actions.

\section{Conclusions}

In this work it is proven, for the class of $N-t h$ gradient continua, that the approach à la Cauchy and the approach à la d'Alembert are absolutely equivalent.

There are several aspects of the studied theory which deserve further investigations. We list here few of them.

1. It is needed to study contact actions on contact surfaces in which there are present curves of discontinuity of Gaussian curvature or its derivatives.

2. Cauchy tetrahedron argument needs to be extended to Cosserat-type continua, where further kinematical descriptors, in addition to placement, are introduced.

3. The weakest regularity assumptions for introduced fields which allow for a Cauchy type representation theorem need to be found.

4. More singular types of wedge shapes need to be introduced and studied as parts of admissible Cauchy cuts.

\section{Acknowledgements}

The authors are fully responsible for the opinions expressed and results presented in the present paper. However they must, also in this occasion, thank G. Maugin for the interest he has always shown in their research. Moreover the interesting discussions with A. Di Carlo were really important for a true understanding of the problems presented by an approach à la Cauchy to second gradient theories: he also attracted their attention to the novel by Borges quoted in one of the previous sections and cited in the references. Indeed the role of A.Di Carlo as "ideas spreader" in the interest of the advancement of science, must be recognized. His activity personifies the metaphor of the Bacon's bee (see e.g. Muntersbjom (2003)), or (using an expression from the ancient greek) of the true scientific "melitta" i.e. the honey bee who spreads the pollen of science from flower to flower. 


\subsection{REFERENCES}

\section{Bibliography}

[1] R. K.Abu Al-Rub Modeling the interfacial effect on the yield strength and flow stress of thin metal films on substrates Mechanics Research Communications 35 65-72 (2008)

[2] R. Abeyaratne,N. Triantafyllidis, An investigation of localization in a porous elastic material using homogenization theory.Trans. ASME J. Appl. Mech. 51, no. 3, 481-486 (1984)

[3] R. Abraham, J.E. Marsden, and T. Ratiu, 'Manifolds, Tensor Analysis, and Applications', Applied Mathematical Sciences, 75, Springer Verlag, (1988).

[4] Alibert, J.-J., Seppecher, P., dell'Isola, F. Truss modular beams with deformation energy depending on higher displacement gradients. Math. Mech. Solids 8 , no. 1, 51-73 (2003).

[5] V.I. Arnold, Mathematical Methods of Classical Mechanics Springer Verlag (1979)-(1989)

[6] C. Banfi, A. Marzocchi and A. Musesti On the principle of virtual powers in continuum mechanics Ricerche di Matematica 55: 299 $310(2006)$

[7] S.Bardenhagen, N. Triantafyllidis Derivation of higher order gradient continuum theories in 2,3-D nonlinear elasticity from periodic lattice models. J. Mech. Phys. Solids 42 , no. 1, 111-139 (1994)

[8] E. Benvenuto La scienza delle costruzioni e il suo sviluppo storico Sansoni, Firenze, (1981)

[9] V. Berdichevsky, Variational Principles of Continuum Mechanics, Springer, (2009).

[10] J.L. Bleustein, A note on the boundary conditions of Toupin's strain-gradient theory International Journal of Solids and Structures, 3 (6), pp. 1053-1057. (1967)

[11] B. Bourdin, G.A. Francfort and J.-J. Marigo, The variational approach to fracture, J. Elasticity, 91, 1-3, 2008, 1-148 (also appeared as a Springer book: ISBN: 978-1-4020-6394-7).

[12] J.L.Borges, Pierre Menard Author of the Quixote (translation by James E. Irby) http://www.coldbacon.com/writing/borgesquixote.html

[13] P. Casal, et H.Gouin, 'Relation entre l'équation de l'énergie et l'équation du mouvement en théorie de Korteweg de la capillaritè', C. R. Acad. Sci. Paris, t. 300, Série II, N. 7 231-233 (1985). 
[14] P.Casal, 'La théorie du second gradient et la capillarité', $C . R$. Acad. Sci. Paris, t. 274, Série A 1571-1574 (1972).

[15] P.Casal, La capillarité interne, Cahier du groupe Français de rhéologie, CNRS VI, 3, pp. 31-37 (1961).

[16] P. Casal, et H. Gouin, 'Relation entre l'équation de l'énergie et l'équation du mouvement en théorie de Korteweg de la capillarité', C. R. Acad. Sci. Paris, t. 300, Série II, N. 7 231-233 (1985).

[17] A. Carcaterra, A. Akay, I.M. Koc Near-irreversibility in a conservative linear structure with singularity points in its modal density Journal of the Acoustical Society of America 1194 2141-2149 (2006)

[18] A. Carcaterra Ensemble energy average and energy flow relationships for nonstationary vibrating systems Journal of Sound and Vibration 288, 3, 751-790 (2005)

[19] F.Collin, R.Chambon and R.Charlier A finite element method for poro mechanical modelling of geotechnical problems using local second gradient models. Int. J. Num. Meth. Engng. 65, 1749-1772 (2006).

[20] G. Colonnetti, Scienza delle costruzioni, Torino, Edizioni scientifiche Einaudi, $3^{\circ}$ ed., (1953-57).

[21] E. Cosserat and F. Cosserat Note sur la théorie de l'action euclidienne. Paris, Gauthier-Villars, (1908).

[22] E. Cosserat, and F. Cosserat Sur la Théorie des Corps Déformables, Herman, Paris, (1909).

[23] N. Daher, G. A.Maugin Virtual power and thermodynamics for electromagnetic continua with interfaces. J. Math. Phys. 27, no. 12, 3022-3035 (1986).

[24] N.Daher, G. A. Maugin The method of virtual power in continuum mechanics. Application to media presenting singular surfaces and interfaces. Acta Mech. 60, no. 3-4, 217-240, (1986)

[25] F. dell'Isola and W. Kosinski "Deduction of thermodynamic balance laws for bidimensional nonmaterial directed continua modelling interphase layers," Archives of Mechanics, vol. 45, , pp. 333-359 (1993).

[26] F. dell'Isola and P. Seppecher, "The relationship between edge contact forces, double force and interstitial working allowed by the principle of virtual power", Comptes Rendus de l'Academie de Sciences - Serie IIb: Mecanique, Physique, Chimie, Astronomie, vol. 321, , pp. 303-308 (1995).

[27] F. dell'Isola and P. Seppecher, "Edge Contact Forces and QuasiBalanced Power", Meccanica, vol. 32, pp. 33-52 (1997) 
[28] F. dell'Isola, G. Sciarra, and S.Vidoli, Generalized Hooke's law for isotropic second gradient materials. Proc. R. Soc. Lond. Ser. A Math. Phys. Eng. Sci. 465, no. 2107, 2177-2196 (2009)

[29] F.dell'Isola, G. Sciarra, R. C.Batra, Static deformations of a linear elastic porous body filled with an inviscid fluid. Essays and papers dedicated to the memory of Clifford Ambrose Truesdell III. Vol. III. J. Elasticity 72, no. 1-3, 99-120 (2003)

[30] F.dell'Isola, M. Guarascio, K. Hutter A variational approach for the deformation of a saturated porous solid. A secondgradient theory extending Terzaghi's effective stress principle. Arch. Appl. Mech. 70, 323-337 (2000)

[31] A. Di Carlo e A. Tatone (Iper-)Tensioni \& Equi-Potenza AIMETA'01 XV Congresso AIMETA di Meccanica Teorica e Applicata 15th AIMETA Congress of Theoretical and Applied Mechanics 2001

[32] M. Degiovanni, A. Marzocchi and A. Musesti Edge-force densities and second-order powers Annali di Matematica 185, 81-103 (2006)

[33] M. Degiovanni, A. Marzocchi, and A. Musesti, A., 'Cauchy fluxes associated with tensor fields having divergence measure', Arch. Ration. Mech. Anal. 147 197-223 (1999).

[34] J.E. Dunn, and J.Serrin, 'On the thermomechanics of interstitial working', Arch. Rational Mech. Anal., 88 (2) 95-133 (1985).

[35] J.E. Dunn 'Interstitial working and a non classical continuum thermodynamics', In: J. Serrin (Ed), New Perspectives in Thermodynamics, Springer Verlag, Berlin, , pp. 187-222 (1986).

[36] G. E. Exadaktylos, I. Vardoulakis, Microstructure in linear elasticity and scale effects: a reconsideration of basic rock mechanics and rock fracture mechanics, Tectonophysics, Volume 335, Issues 1-2, 25 June 2001, Pages 81-109,

[37] A.C. Fannjiang, Y.S. Chan, and G.H. Paulino Strain gradient elasticity for antiplane shear cracks: a hypersingular integrodifferential equation approach. SIAM J. Appl. Math. 62 , no. 3, 1066-1091 (electronic) (2001/02).

[38] S. Forest, M. Amestoy, S. Cantournet, G. Damamme, S. Kruch Mécanique des Milieux Continus ECOLE DES MINES DE PARIS Année 2005-2006

[39] S. Forest Mechanics of generalized continua: construction by homogenization J.Phys. IV France 81998

[40] S. Forest Homogenization methods and the mechanics of generalized continua - part 2 Theoretical and applied Mechanics vol. 28-29, pp. 113-143 (2002) 
[41] S. Forest. Milieux continus généralisés et matériaux hétérogènes. Les Presses de l'Ecole des Mines de Paris, ISBN : 2911762-67-3, 200 pages, (2006).

[42] S. Forest. Generalized continua. In K.H.J. Buschow, R.W. Cahn, M.C. Flemings, B. Ilschner, E.J. Kramer, and S. Mahajan, editors, Encyclopedia of Materials : Science and Technology updates, pages 1-7. Elsevier, Oxford, (2005).

[43] S. Forest and M. Amestoy. Mécanique des milieux continus. Cours de l'Ecole des Mines de Paris n25e6 3121, 264 pages, (2004, 2005, 2006).

[44] S. Forest. Milieux continus généralisés et matériaux hétérogènes. Mémoire d'habilitation à diriger des recherches, (2004).

[45] E. Fried and M.E. Gurtin, Tractions, balances, and boundary conditions for nonsimple materials with application to liquid flow at small length scales. Archive for Rational Mechanics and Analysis 182 , 513-554 (2006).

[46] E. Fried and M.E. Gurtin, A continuum mechanical theory for turbulence: a generalized Navier -Stokes-equation with boundary conditions. Theoretical and Computational Fluid Dynamics $182,513-554$ (2008)

[47] P. Germain Cours de Mècanique des Milieux Continus, tome I, Masson, Paris, (1973).

[48] P.Germain La méthode des puissances virtuelles en mécanique des milieux continus. Première partie. Théorie du second gradient. J. Mécanique 12, 235-274 (1973)

[49] P. Germain The method of virtual power in continuum mechanics. Part 2: Microstructure. SIAM J. Appl. Math. 25, 556$575(1973)$

[50] P. Germain 'Sur l'application de la méthode des puissances virtuelles en mécanique des milieux continus', C. R. Acad. Sci. Paris Série A-B 274 A1051-A1055.(1972)

[51] A. E. Green, R. S. Rivlin, , Multipolar continuum mechanics, Arch. Rational Mech. Anal., 17, 113-147 (1964)

[52] A. E. Green, R. S.Rivlin, Simple force and stress multipoles, Arch. Rational Mech. Anal.,16, 325-353 (1964)

[53] A. E. Green, R. S. Rivlin, .On Cauchy's equations of motion, $Z$. Angew. Math. Phys.,ZAMP. 15,, 290-292, (1964)

[54] A. E. Green, R. S. Rivlin Multipolar continuum mechanics: Functional theory. I, Proc. Roy. Soc. Ser. A, 284, 303-324 (1965)

[55] M.E. Gurtin, A gradient theory of single-crystal viscoplasticity that accounts for geometrically necessary dislocations, International Journal of Plasticity 50 , 809-819 (2002). 
[56] N. Kirchner, P. Steinmann, On the material setting of gradient hyperelasticity. (English summary) Math. Mech. Solids 12 (2007)

[57] O.D. Kellogg, , Foundations of Potential Theory Springer, Berlin, (1929)

[58] W. Kosinski, Field Singularities and Wave Analysis in Continuum Mechanics. Ellis Horwood Series: Mathematics and Its Applications, Wiley \& Sons, PWN - Polish Scientific Publishers, Warsaw 1986 ,

[59] R. Larsson, S. A Diebels, Second-order homogenization procedure for multi-scale analysis based on micropolar kinematics. Internat. J. Numer. Methods Engrg. 69 , no. 12, 2485-2512 (2007).

[60] M. Lazar, G. A. Maugin A note on line forces in gradient elasticity Mechanics Research Communications 33 674-680 (2006)

[61] M. Lucchesi ·M. Silhavý ·N. Zani On the Balance Equation for Stresses Concentrated on Curves J Elasticity 90:209-223 (2008).

[62] A. Madeo, F. dell'Isola, N. Ianiro, G. Sciarra, "A second gradient poroelastic model of consolidation", SIMAI 2008, Rome $15-19$ September (2008).

[63] A. Madeo, F. dell'Isola, N. Ianiro, and G. Sciarra, "A variational deduction of second gradient poroelasticity II: An application to the consolidation problem," Journal of Mechanics of Materials and Structures, vol. 3, , pp. 607-625 (2008)

[64] A.Marzocchi,, A.Musesti, Balanced virtual powers in Continuum Mechanics. Meccanica 38, 369-389 (2003)

[65] A. Marzocchi, A.Musesti,, 'Decomposition and integral representation of Cauchy interactions associated with measures', Cont. Mech. Thermodyn. 13 149-169 (2001) .

[66] G. Maugin MathReview MR1437786 (98d:73003) 73A05 (73B18 73S10) on the paper F.dell'Isola P. Seppecher (1997).

[67] G. Maugin MathReview MR1600928 (99e:73005) on the paper G. Capriz and G. Mazzini Invariance and balance in continuum mechanics.Nonlinear analysis and continuum mechanics (Ferrara,1992), 2735, Springer, New York, 1998.

[68] G. A.Maugin A.V. Metrikine Editors Mechanics of Generalized Continua, One Hundred Years After the Cosserats, Springer (2010)

[69] R.D. Mindlin, Second gradient of strain and surface tension in linear elasticity Int. J. Solids and Struct. 1,4,417-438 (1965)

[70] R.D. Mindlin, Micro-structure in linear elasticity, Arch. Rat. Mech. Analysis, , vol. 16, pp. 51-78 (1964) 
[71] R. D. Mindlin, Complex representation of displacements and stresses in plane strain with couple-stresses. 1965 Appl. Theory of Functions in Continuum Mechanics (Proc. Internat. Sympos ., Tbilisi), Vol. I, Mechanics of Solids (Russian) pp. 256-259 Izdat. "Nauka", Moscow (1963)

[72] R. D. Mindlin, H. F.Tiersten, Effects of couple-stresses in linear elasticity. Arch. Rational Mech. Anal. 11 415-448 (1962)

[73] R. D. Mindlin Influence of couple-stresses on stress concentrations Main features of cosserat theory are reviewed by lecturer and some recent solutions of the equations, for cases of stress concentration around small holes in elastic solids, are described EXPERIMENTAL MECHANICS 3, 1, 1-7, THE WILLIAM M. MURRAY LECTURE, (1962)

[74] R. D. Mindlin N. N. Eshel On first strain-gradient theories in linear elasticity International Journal of Solids and Structures 4, 1 1968, 109-124

[75] R.D. Mindlin, Stress functions for a Cosserat continuum International Journal of Solids and Structures, 1 (3), pp. 265-271 (1965)

[76] R.D.Mindlin On the equations of elastic materials with microstructure International Journal of Solids and Structures, 1 (1), pp. 73-78 (1965).

[77] M. Muntersbjom, Francis Bacon's Philosophy of Science: Machina intellectus and Forma indita Philosophy of Science, 70 (December 2003) pp. 1137-1148.

[78] W. Noll, E.G.,Virga, 'On edge interactions and surface tension', Arch. Rational Mech. Anal., 111 (1) 1-31 (1990).

[79] W. Noll 'The foundations of classical mechanics in the light of recent advances in continuum mechanics' Proceeding of the Berkeley Symposium on the Axiomatic Method, Amsterdam, , pp. 226-281 (1959).

[80] W. Noll 'Lectures on the foundations of continuum mechanics and thermodynamics', Arch. Rational Mech. Anal. 52 62-92 (1973).

[81] W. Noll 'The geometry of contact separation and reformation of continuous bodies', Arch. Rational Mech. Anal., 122 (3) 197-212 (1993).

[82] C. Pideri and P. Seppecher A second gradient material resulting from the homogenization of an heterogeneous linear elastic medium. Contin. Mech. Thermodyn. 9, no. 5, 241-257 (1997).

[83] P. Podio-Guidugli A virtual power format for thermomechanics Continuum Mech. Thermodyn. 20: 479-487 (2009) 
[84] P. Podio-Guidugli, Contact interactions, stress, and material symmetry, for nonsimple elastic materials. (English, SerboCroatian summary) Issue dedicated to the memory of Professor Rastko Stojanovic (Belgrade, 2002). Theoret. Appl. Mech. 28/29 (2002), 261-276.

[85] P. Podio-Guidugli and M. Vianello Hypertractions and hyperstresses convey the same mechanical information Continuum Mech. Thermodyn. 22:163-176 (2010)

[86] C. Polizzotto, Strain-gradient elastic-plastic material models and assessment of the higher order boundary conditions. Eur. J. Mech. A Solids 26 , no. 2, 189-211 (2007).

[87] Rorres C. Completing Book II of Archimedes's On Floating Bodies THE MATHEMATICAL INTELLIGENCER 26,3, Pages 32$42(2004)$

[88] L. Russo The Forgotten Revolution Springer Verlag (2003)

[89] J. Salençon Mécanique des milieux continus Ed. Ellipses (1988)(1995) Handbook of Continuum Mechanics Ed. Springer (Berlin, 2001) Mécanique des milieux continus. Tome I. Éd. École polytechnique, Palaiseau ; Ellipses, Paris, (2002)-(2005)

[90] L. Schwartz, Théorie des Distributions, Hermann Paris, (1973).

[91] G. Sciarra, F. dell'Isola and O. Coussy Second gradient poromechanics. Internat. J. Solids Structures 44 , no. 20, 6607-6629 (2007).

[92] G. Sciarra, F. dell'Isola and K. Hutter A solid-fluid mixture model allowing for solid dilatation under external pressure. Contin. Mech. Thermodyn. 13 , no. 5, 287-306 (2001).

[93] G. Sciarra, F. dell'Isola, N. Ianiro, and A. Madeo, "A variational deduction of second gradient poroelasticity part I: General theory", Journal of Mechanics of Materials and Structures, vol. 3, , pp. 507-526 (2008).

[94] P. Seppecher 'Etude des conditions aux limites en théorie du second gradient: cas de la capillarité, C. R. Acad. Sci. Paris, t. 309, Série II 497-502 (1989).

[95] P. Seppecher Etude d'une Modélisation des Zones Capillaires Fluides: Interfaces et Lignes de Contact, Thèse de l'Université Paris VI, Avril (1987).

[96] M. Śilhavý 'The existence of the flux vector and the divergence theorem for general Cauchy fluxes', Arch.Ration. Mech. Anal. 90 195-211 (1985).

[97] M. Šilhavý 'Cauchy's stress theorem and tensor fields with divergences in Lp', Arch. Ration. Mech. Anal. 116 223-255 (1991). 
[98] M. Sokolowski, Theory of couple-stresses in bodies with constrained rotations. In CISM courses and lectures, vol. 26. Berlin, Germany: Springer (1970).

[99] M. Spivak A comprehensive introduction to differential geometry. Voll. I and II. Second edition. Publish or Perish, Inc., Wilmington, Del. (1979)

[100] A. S. J. Suiker and C. S. Chang, Application of higher-order tensor theory for formulating enhanced continuum models. Acta Mech. 142, 223-234 (2000).

[101] R.A. Toupin Elastic Materials with couple-stresses, Arch. Rat. Mech. Analysis, , vol. 11, pp. 385-414 (1962)

[102] R. A. Toupin, Theories of elasticity with couple-stress. Arch. Rational Mech. Anal. 17 85-112 (1964).

[103] N. Triantafyllidis and S. Bardenhagen, On higher order gradient continuum theories in 1-D nonlinear elasticity. Derivation from and comparison to the corresponding discrete models. J. Elasticity 33 (1993)

[104] N. Triantafyllidis and S. Bardenhagen The influence of scale size on the stability of periodic solids and the role of associated higher order gradient continuum models. J. Mech. Phys. Solids 44 , no. 11, 1891-1928 (1996)

[105] C.A.Truesdell A First Course in Rational Continuum Mechanics, Vol. I General Concepts, Academic Press, New York, 1977.

[106] N. Triantafyllidis and E.C. Aifantis A gradient approach to localization of deformation. I. Hyperelastic materials. J. Elasticity 16 , no. 3, 225-237 (1986).

[107] Y.Yang, and A. Misra "Higher-order stress-strain theory for damage modeling implemented in an element-free Galerkin formulation," Computer Modeling in Engineering and Sciences, Vol. 64, No. 1, 1-36 (2010)

[108] Y. Yang, W.Y. Ching and Misra, A.,"Higher-order continuum theory applied to fracture simulation of nano-scale intergranular glassy film," Journal of Nanomechanics and Micromechanics, (in print). (2011)

[109] G. Vailati, Il principio dei lavori virtuali da Aristotele a Erone d'Alessandria, Scritti (Bologna, Forni, 1987), vol. II, pp. 113-128, Atti della R. Accademia delle Scienze di Torino, vol. XXXII, adunanza del 13 giugno 1897, quaderno IG (091) 75 I - III (1897). 